Gutenberg School of Management and Economics Discussion Paper Series

\title{
In-Depth Analysis of Pricing Problem Relaxations for the Capacitated Arc-Routing Problem
}

Claudia Bode and Stefan Irnich

November 2012

Discussion paper number 1212

Johannes Gutenberg University Mainz

Gutenberg School of Management and Economics

Jakob-Welder-Weg 9

55128 Mainz

Germany

$\underline{\text { wiwi.uni-mainz.de }}$ 
Contact details

Claudia Bode

Chair of Logistics Management

Johannes Gutenberg University Mainz

Jakob-Welder-Weg 9

55128 Mainz

Germany

claudia.bode@uni-mainz.de

Stefan Irnich

Chair of Logistics Management

Gutenberg School of Management and Economics

Johannes Gutenberg University Mainz

Jakob-Welder-Weg 9

55128 Mainz

Germany

irnich@uni-mainz.de

All discussion papers can be downloaded from http://wiwi.uni-mainz.de/DP 


\title{
In-Depth Analysis of Pricing Problem Relaxations for the Capacitated Arc-Routing Problem
}

\author{
Claudia Bode, Stefan Irnich \\ Chair of Logistics Management, Gutenberg School of Management and Economics, \\ Johannes Gutenberg University Mainz, Jakob-Welder-Weg 9, D-55128 Mainz, Germany.
}

\begin{abstract}
Recently, Bode and Irnich ('Cut-First Branch-and-Price-Second for the Capacitated Arc-Routing Problem', Operations Research, 2012, doi: 10.1287/opre.1120.1079) presented a cut-first branch-and-price-second algorithm for solving the capacitated arc-routing problem (CARP). The fundamental difference to other approaches from the literature for exactly solving the CARP is that the entire algorithm works directly on the typically sparse underlying graph representing the street network. This enables the use of highly efficient dynamic programming-based pricing algorithms for solving the column-generation subproblem also known as the pricing problem. The contribution of this paper is the in-depth analysis of the CARP pricing problem and its possible relaxations, including the construction of new labeling algorithms for their solution, theoretical complexity results, and comprehensive computational tests on standard benchmark problems. We will show that a systematic variation of different relaxations provides a powerful approach to solve knowingly hard instances of the CARP to proven optimality.
\end{abstract}

Key words: CARP, column generation, branch-and-price, pricing problem, relaxations

\section{Introduction}

The capacitated arc-routing problem (CARP) is the fundamental multiple-vehicle arc-routing problem with applications in waste collection, postal delivery, winter services and more (Dror, 2000, Corberán and Prins, 2010). Recently, Bode and Irnich (2012) presented a new exact solution approach based on an aggregated, non-symmetric formulation that was derived via a Dantzig-Wolfe decomposition of the well-known two-index formulation (Belenguer and Benavent, 1998). For its solution, violated valid inequalities as well as missing variables are generated dynamically. The corresponding cut-and-column-generation algorithm as a whole exploits the fact that the underlying CARP graph is sparse (exploitation of sparsity is an idea that was originally coined by Letchford and Oukil (2009)). Note that any approach using a transformation of the CARP into a node-routing problem results in dense graphs (Baldacci and Maniezzo, 2006; Longo et al. 2006: Bartolini et al. 2012). Using the one-index formulation of the CARP, some relevant valid inequalities are computed a priori in the initial cutting phase. This provides a very fast warm-start of the columngeneration process. Due to direct use of a sparse network for fast pricing, the proposed column-generation algorithm often produces strong lower bounds in relatively short computation time for many instances from the literature. Integrated into branch-and-bound, the approach becomes a cut-first branch-and-price-second algorithm. The computation of integer solutions then benefits from the non-symmetric formulation and, in particular, from an effective branching scheme.

The contribution of this paper is the in-depth analysis of the CARP pricing problem and its possible relaxations, including the construction of new labeling algorithms for their solution, theoretical complexity results, and comprehensive computational tests on standard benchmark problems. Using pricing problem

Email addresses: claudia.bode@uni-mainz.de (Claudia Bode), irnich@uni-mainz.de (Stefan Irnich) 
relaxations is a standard technique in column generation (Lübbecke and Desrosiers, 2005; Desaulniers et al. 2005 because pricing problems in routing applications are typically strongly $\mathcal{N} \mathcal{P}$-hard elementary shortestpath problems with resource constraints (ESPPRC, Irnich and Desaulniers, 2005). In fact, many successful column-generation approaches play with the trade-off that different pricing problems relaxations offer (Irnich and Villeneuve, 2006, Baldacci et al., 2011b). Stronger relaxations produce tighter lower bounds, but come at the cost of being harder to solve leading to longer computation times in the pricing subproblem. The branch-and-price approach in (Bode and Irnich, 2012) made use of just one relaxation producing 2-loops free tours (Benavent et al., 1992). This relaxation is particularly beneficial because it is compatible and at the same time indispensable for branching on followers. Actually, branching on followers and non-followers is the only effective technique known to guarantee the integrality in branch-and-price when pricing is performed on the original sparse network.

Bode and Irnich (2012) already showed that pricing relaxations based on $k$-loop elimination produce better root node lower bounds. However, for these and other possible relaxations it remained unclear how integer solutions can be computed using the aforementioned branching scheme. This paper is intended to fill the gap by showing how different pricing relaxations can be made compatible with the requirements imposed by branching. We will discuss and empirically analyze the trade-offs between hardness of pricing and strength of lower bounds for various pricing relaxations. As a result, we are able to compute new best lower bounds and optimal solutions for several knowingly hard CARP instances from the benchmark sets of Eglese and Li (1992), Brandão and Eglese (2008), and Beullens et al. (2003).

The remainder of this paper is structured as follows: The next section defines the CARP and briefly summarizes the cut-first branch-and-price-second approach presented in (Bode and Irnich, 2012). Section 3 presents the pricing problem, and discusses well-known and also new pricing relaxations. Several acceleration techniques for solving the shortest-path subproblems via dynamic-programming labeling algorithms such as bidirectional pricing, bounding, and scaling are summarized and adapted to the new relaxations in Section 4. In Section 5. we presents comprehensive computational results and final conclusions are drawn in Section 6 .

\section{Cut-First Branch-and-Price-Second for the CARP}

The CARP has been introduced by Golden and Wong (1981) and studied intensively both from a heuristic and exact algorithm point of view. Heuristics and metaheuristics are essential for computing good upper bounds. Some prominent and successful approaches from the literature include approaches based on tabu search (Brandão and Eglese, 2008), genetic or memetic algorithms (Lacomme et al., 2001, Fu et al., 2010), guided local search (Beullens et al. 2003), variable neighborhood search (Polacek et al., 2008), ant colony optimization (Santos et al. | 2010), and many more. A survey on heuristic methods is (Prins, 2013). On the other hand, there are several approaches for computing good lower bounds. Pure polyhedral approaches to the CARP are discussed in (Letchford, 1997; Belenguer and Benavent, 1998, 2003, Ahr, 2004). At the moment, it seems that the most successful exact solution approaches are all based on a combination of cut-and-column generation. Gómez-Cabrero et al. (2005) and Martinelli et al. (2011a) proposed column generation-based algorithms, where either initially computed cuts are added to the column-generation master program or a cutting-plane algorithm is applied during and after the column-generation process. Thereafter, a branch-and-bound procedure follows in (Martinelli et al. 2011a). Their branching scheme is not complete meaning that they can only guarantee integer deadheading flows, but route variables may remain fractional.

Complete exact methods were recently presented in (Bartolini et al., 2012, Bode and Irnich, 2012). The first method consists of computing a cascade of non-decreasing lower bounds, enumerating all routes with reduced cost smaller than the integrality gap of upper bound minus the best lower bound, and finally solving the master program with a (general purpose) mixed integer-programming solver. Note that Bartolini et al. (2012) make intensive use of a transformation of the CARP into a generalized vehicle-routing problem (GVRP) so that route generation is performed on a dense graph. In contrast, the sparsity of the CARP network is heavily exploited by Bode and Irnich (2012), where in the first phase a cutting-plane algorithm is applied to initialize the column-generation master program and in the second phase the branch-and-price algorithm is executed. This general approach will be explained in detail in Sections 2.2 and 2.3 . 
A comprehensive overview on exact CARP approaches is given in (Belenguer et al., 2013) and recent surveys on both heuristic and exact approaches are (Wøhlk, 2008; Corberán and Prins, 2010).

\subsection{Notation and Definition of the CARP}

For the formal definition of the CARP, we assume an undirected and simple graph $G=(V, E)$ with node set $V$ and edge set $E$. In applications, this graph $G$ is typically sparse so that $|E| \leq \Delta|V|$ holds for a small number $\Delta>0$. A distinguished node $d \in V$ is given representing the depot. All edges $e \in E$ have an associated non-negative integer demand $q_{e} \geq 0$ and those with positive demand form the subset $E_{R} \subseteq E$ of required edges. Required edges have to be served exactly once. All edges $e \in E$, either required or not, can be traversed without providing service (=deadheading). CARP costs consist of two components, that is, service costs $c_{e}^{\text {serv }}$ for servicing required edges $e$ and deadheading costs $c_{e}$ for all edges $e$ deadheaded.

A tour is an Eulerian subgraph $\left(V^{\prime}, E^{\prime}\right)$ of $G$ with $V^{\prime} \subseteq V$ and $E^{\prime} \subseteq E$, where $d \in V^{\prime}$ holds and $E^{\prime}$ may contain copies of edges. In fact, $E^{\prime}$ is a multi-set. By definition, a Eulerian subgraph is connected and all its nodes have an even and positive node degree. A feasible tour serves a subset $E_{s} \subseteq E^{\prime}$ with demand $\sum_{e \in E_{s}} q_{e}$ not exceeding the vehicle capacity $C$. It is assumed that all other edges $E_{d}:=E^{\prime} \backslash E_{s}$ are deadheaded (counting copies appropriately). Moreover, it must be elementary meaning that $E_{s}$ is a simple set and does not contain copies of parallel edges. An optimal CARP solution is a cost-minimal set of feasible tours such that every required edge $e \in E_{R}$ is serviced by exactly one tour. Note that there might exist a huge number of Eulerian paths for a given Eulerian subgraph, i.e., the same feasible tour might be represented by several possibilities of traversals.

Some authors define the CARP for an unlimited fleet of vehicles (Belenguer and Benavent, 2003, Longo et al. , 2006, Bartolini et al. 2012), others fix the number of vehicles (Bode and Irnich, 2012, Belenguer and Benavent, 1998). Here, the fleet size is also fixed to the minimum number $K$ of required vehicles (computed by solving a bin-packing problem) and we assume that each vehicle of the homogeneous fleet has capacity $C$ and is stationed at the depot $d$.

Throughout this paper, we use the following standard notation: Given a subset $S \subseteq V$, the cut set $\delta(S)$ (the set $E(S)$ ) is the set of edges with exactly one (both) endpoint(s) in $S$. The subscript ${ }_{R}$ indicates the restriction to subsets of required edges so that $\delta_{R}(S)=\delta(S) \cap E_{R}$ and $E_{R}(S)=E(S) \cap E_{R}$ holds. For simplicity, the abbreviation $\delta(i)$ is used instead of $\delta(\{i\})$ (also $\delta_{R}(i)$ for $\delta_{R}(\{i\})$ ). Given a subset $F \subseteq E$ and any parameter or variable $y$, the term $y(F)$ stands for $\sum_{e \in F} y_{e}$.

\subsection{Cutting-Plane Generation: First Phase}

The first phase of the algorithm presented in (Bode and Irnich, 2012) consists of the generation of a relevant set of valid inequalities that are later added to the column-generation formulation. Solving the following one-index formulation with a cutting-plane procedure, the added inequalities are those that are binding at the end.

The one-index formulation was first considered independently by Letchford (1997) and Belenguer and Benavent (1998). It can be used for computing lower bounds, which are known to be optimal or very tight at least for small and medium-sized instances. However, the one-index formulation is a relaxation of the CARP, since its associated integer polyhedron generally contains infeasible solutions. It uses aggregated deadheading variables $y_{e} \in \mathbb{Z}_{+}$one for each edge $e \in E$. The attribute aggregated refers to the fact that $y_{e}$ counts the deadheadings over edge $e$ performed by all $K$ vehicles together. The one-index formulation reads as follows:

$$
\begin{array}{cl}
\min & c^{\top} y \\
\text { s.t. } & y(\delta(S)) \geq 2 K(S)-\left|\delta_{R}(S)\right| \quad \text { for all } \emptyset \neq S \subseteq V \backslash\{d\} \\
& y(\delta(S)) \geq 1 \quad \text { for all } \emptyset \neq S \subseteq V,\left|\delta_{R}(S)\right| \text { odd } \\
& y \in \mathbb{Z}_{+}^{|E|}
\end{array}
$$

The objective (1) minimizes the costs of all deadheadings (note that service costs are constant and therefore irrelevant for routing decisions). The capacity inequalities (2) require that there are at least $2 K(S)$ traversals 
(services and deadheadings) over the cutset $\delta(S)$. Herein, $K(S)$ is the minimum number of vehicles needed to service the edges $E_{R}(S) \cup \delta_{R}(S)$. The number $K(S)$ can be approximated by $\left\lceil q\left(E_{R}(S) \cup \delta_{R}(S)\right) / C\right\rceil$ and computed exactly by solving a bin-packing problem. Furthermore, the odd-cut inequalities (3) ensure for each subset $S$ with an odd number of required edges in the cut $\delta(S)$ that at least one deadheading is performed. Belenguer and Benavent (2003) introduced disjoint-path inequalities as another class of valid cuts for the CARP. The idea is to consider not only the demand of $E_{R}(S) \cup \delta_{R}(S)$ but also the demand on a path from the depot to the set $S$. The general form of all valid inequalities (including disjoint-path inequalities) can be written as $\sum_{e \in E} d_{e s} y_{e} \geq r_{s}$ for $s \in \mathcal{S}$ where $\mathcal{S}$ is the set of all inequalities and $d_{e s}$ the coefficient of edge $e$ in a particular cut indexed by $s$.

\subsection{Branch-and-Price: Second Phase}

In the second phase of the algorithm presented in (Bode and Irnich, 2012), a restricted master program is iteratively reoptimized and variables with negative reduced costs are generated at each iteration. To obtain integer solutions a branching scheme is applied.

\subsubsection{Master Program}

The master program is derived by a Danzig-Wolfe decomposition from the two-index formulation by Belenguer and Benavent (1998) extended by additional cuts from the first phase. Because a homogeneous fleet of vehicles is assumed, an aggregation over all vehicles is applied. As a result, the column-generation formulation contains two sets of variables. On the one hand, there are variables $\lambda_{r} \geq 0$, one for every efficient feasible route $r \in \Omega$, where efficient means that no deadheading along a cycle in $G$ is performed. On the other hand, variables $z_{e} \geq 0$ for every edge $e=\{i, j\} \in E$ indicate a deadheading along the cycle $(e, e)=(i, j, i)$.

Let $\bar{x}_{e r}$ and $\bar{y}_{e r}$ be the number of times a route $r$ services and deadheads through an edge $e$, respectively. The linear relaxation (MP) of the extensive formulation reads then:

$$
\begin{array}{ll}
\min & \sum_{r \in \Omega} c_{r} \lambda_{r}+\sum_{e \in E}\left(2 c_{e}\right) z_{e} \\
\text { s.t. } & \sum_{r \in \Omega} \bar{x}_{e r} \lambda_{r}=1 \quad \text { for all } e \in E_{R} \\
& \sum_{r \in \Omega} d_{s r} \lambda_{r}+\sum_{e \in E}\left(2 d_{e s}\right) z_{e} \geq r_{s} \quad \text { for all } s \in \mathcal{S} \\
& \mathbf{1}^{\top} \lambda=K \\
& \lambda \geq \mathbf{0}, z \geq \mathbf{0}
\end{array}
$$

The objective (5) consists of minimizing the costs of the routes plus the costs of deadheading along simple cycles. Each required edge must be covered by one route (6). Both route variables $\lambda_{r}$ and cycle variables $z_{e}$ are impacted by the additional cuts from phase one. For a specific cut $s \in \mathcal{S}$, the route $r \in \Omega$ has the coefficient $d_{s r}=\sum_{e \in E} d_{e s} \bar{y}_{e r}$, and the respective coefficient of the cycle variable $z_{e}$ is $2 d_{e s}$. Thus, the general form of cuts from the one-index formulation can be transformed into the reformulated cuts (7). Since the number of vehicles is fixed, exactly $K$ routes are used (8) and all variables are non-negative (9).

Note that the exact integrality condition for the integer master program (IMP) is neither $\lambda \in \mathbb{Z}_{+}^{\Omega}$ and $z \in \mathbb{Z}_{+}^{E}$ nor

$$
y_{e}=\sum_{r \in \Omega} \bar{y}_{e r} \lambda_{r} \in \mathbb{Z}_{+} .
$$

The first condition is sufficient, but not necessary, because integer solution can sometimes be reconstructed from fractional $\lambda$ variables (Bode and Irnich, 2012). The latter conditions (10) are necessary, but not sufficient, see Section 2.3.3 on branching. 


\subsubsection{Pricing Problem}

Because the restricted master program (RMP) is initialized with a proper subset of route variables $\lambda_{r}$, missing variables with negative reduced costs must be priced out. In fact, the task of the pricing problem is the generation of those variables. Let $\pi=\left(\pi_{e}\right)_{e \in E_{R}}$ be the vector of dual prices for covering constraints (6), $\beta=\left(\beta_{s}\right)$ the vector of dual prices for active valid inequalities (7), and $\mu$ the dual price to the generalized convexity constraint (8). Reduced costs for service and deadheading are defined as follows:

$$
\tilde{c}_{e}^{\text {serv }}=c_{e}^{\text {serv }}-\pi_{e} \text { for all } e \in E_{R} \quad \text { and } \quad \tilde{c}_{e}=c_{e}-\sum_{s \in \mathcal{S}} d_{e s} \beta_{s} \text { for all } e \in E .
$$

With binary variables $x_{e}$ for $e \in E_{R}$ indicating service and integer variables $y_{e}$ for $e \in E$ for deadheading, the pricing problem to $(\pi, \beta, \mu)$ is:

$$
\begin{aligned}
z_{P P}(\pi, \beta, \mu)= & \min \tilde{c}^{\text {serv }, \top} x+\tilde{c}^{\top} y-\mu \\
\text { s.t. } \quad & x\left(\delta_{R}(S)\right)+y(\delta(S)) \geq 2 x_{f} \quad \text { for all } S \subseteq V \backslash\{d\}, f \in E_{R}(S) \\
& x\left(\delta_{R}(i)\right)+y(\delta(i))=2 p_{i} \quad \text { for all } i \in V \\
& q^{\top} x \leq C \\
& p \in \mathbb{Z}_{+}^{|V|}, x \in\{0,1\}^{\left|E_{R}\right|}, y \in \mathbb{Z}_{+}^{|E|}
\end{aligned}
$$

The objective $\sqrt{12}$ ) is the minimization of the reduced costs. Constraints $(13)$ ensure connectivity of all required edges serviced. An even node degree is guaranteed by $(14)$ using auxiliary integer variables $p_{i}$, one for each node $i \in V$. Constraint $[15)$ is the capacity constraint.

Obviously, whenever deadheading gives no profit, i.e., $\tilde{c}_{e} \geq 0$ for all $e \in E$, it is not efficient to have cycles consisting only of deadheading. However, the two-index formulation, from which Bode and Irnich (2012) derived the master program and pricing problem, allows deadheading cycles denoted as extended $k$-routes in (Belenguer and Benavent, 1998). These extended $k$-routes correspond to extreme rays of the polyhedron formed by (13)-(16). The variables $z_{e}$ in the master program (5)-(9) model cycles $(e, e)=(i, j, i)$ for each edge $e=\{i, j\} \in E$. Additional variables in this master problem (the primal problem) correspond to inequalities in the associated dual problem. Therefore, the variables $z_{e}$ give dual inequalities of the form $\sum_{s \in \mathcal{S}} d_{e s} \beta_{s} \leq c_{e}$ for all $e \in E$. These dual inequalities result in a stabilization of the dual variables $\beta_{s}$ (Ben Amor et al. , 2006). Moreover, the algorithmic advantage for pricing is the guarantee that the reduced $\operatorname{costs} \tilde{c}_{e}$ of deadheadings over all edges are non-negative. The algorithms presented in Section 3 substantially rely on that property.

Note that optimal CARP tours require only the knowledge of the Eulerian subgraphs $\left(V^{\prime}, E^{\prime}\right)$ and the partition of $E^{\prime}$ into served edges $E_{s}=\left\{e \in E: x_{e}=1\right\}$ and deadheaded edges $E_{d}$. The pricing problem is in fact not a routing problem, since the ordering of serviced and deadheading edges is irrelevant. However, the only viable approach known to us for solving the pricing problem is to compute paths. Hence, we solve a routing problem and herewith determine an ordering of serviced and deadheading edges. We will see that this ordering is also crucial for the branching scheme presented in the next section. As pointed out earlier by Bartolini et al. (2012), a feasible CARP tour can then be represented by several possibilities of traversing the corresponding Eulerian subgraph.

Summarizing, the pricing problem asks for a feasible CARP tour with minimum reduced cost, where reduced cost $\tilde{c}_{e}^{\text {serv }}$ and $\tilde{c}_{e}^{\text {deadh }}$ for servicing and deadheading along each edge $e \in E$ are given. Since service variables $x_{e}$ are binary, no feasible CARP tour can perform a service for an edge more than once. This is exactly the definition of an elementary CARP tour. Relaxing the elementarity constraint leads to easier solvable subproblems at the cost of a generally weakened master program lower bound.

\subsubsection{Branching}

In order to obtain integer solutions, a hierarchical branching scheme was devised. It consists of three levels of branching decisions: (1) branching on node degrees, whenever a node with a non-even degree exists, (2) branching on edges with fractional edge flow, (3) branching on follower information, whenever 
the information if two edges are serviced consecutive is fractional. Note that the third branching decision is applicable, since the pricing problem is solved as a routing problem, where an ordering of serviced edges is determined. This decision guarantees integer route variables and can be handled by modifying the underlying pricing network. Bode and Irnich (2012) showed that follower constraints in the branching part can be handled in the pricing problem by adding edges that represent certain paths. On the other hand, nonfollower constraints are handled by associating the same task to the corresponding edges. Combinations of several follower and non-follower constraints are more intricate to implement, but follow the same idea.

\section{Pricing Problem Relaxations}

Letchford and Oukil (2009) analyzed two mixed integer linear programming (MIP) models for solving the elementary pricing problem $(12)-(16)$. When solved with the general purpose MIP solver CPLEX, the resulting computation times were prohibitively long. In principle, the pricing problem (12)-(16) is solvable as an ESPPRC with tasks on service edges using known labeling techniques from the literature (see Irnich and Desaulniers, 2005). However, as paths can become rather long, ESPPRC labeling still suffers from extensive computation times.

Since the ESPPRC is strongly $\mathcal{N} \mathcal{P}$-hard, different relaxations were considered in the literature. Letchford and Oukil (2009) proved that the non-elementary relaxation of the pricing problem can be solved in pseudopolynomial time $\mathcal{O}(C(|E|+|V| \log |V|))$. Their labeling algorithm comprises two building blocks invoked alternately, one is similar to standard labeling approaches for extending labels along service edges and the other is a Dijkstra-like algorithm for extensions along deadheading edges. The Dijkstra steps rely on the property that deadheading edges have non-negative reduced costs (this can be assured, see Section 2.3.2).

A stronger formulation than the non-elementary SPPRC results from the 2-loop-free (=task-1-cycle-free) pricing relaxation already known for the CARP from the work of Benavent et al. (1992). Note that task2-loop-free pricing in the arc routing context allows paths containing task sequences of the form $(a, b, a)$, whereas $(a, a)$ is forbidden. However, in the node routing context node-2-cycle-free pricing allows subpaths $(i, j, k, i)$ and forbids $(i, j, i)$. Both strategies have in common requiring two paths to dominate a third one (see Section 3.4 for further details) so that one must record, for every state, a best and a second best label having a different last task. To distinguish between arc and node routing, we will always refer to loop freeness in the arc-routing context. Comprehensive computational results with 2-loop-free tours were already presented in (Bode and Irnich, 2012).

General requirements. We will now outline requirements on any relaxation of the pricing problem to be used within the presented branch-and-price algorithm. In general, applying the suggested hierarchical branching scheme with branching on non-follower constraints means that any pricing problem relaxation must be able to handle two sets of tasks:

- tasks $\mathcal{T}^{E}$ for modeling the elementary routes

- tasks $\mathcal{T}^{B}$ for respecting non-follower constraints imposed by branching (2-loop-free tours)

The set $\mathcal{T}^{E}$ models elementary routes, and due to network modifications in the branching phase, there can be no, one or several tasks of $\mathcal{T}^{E}$ (forming a task sequence) on a single edge. More precisely, edges modeling deadheading have no task, the original service edges $e \in E_{R}$ have one task, and edges representing longer paths have a task sequence.

By introducing another set $\mathcal{T}^{B}$ of tasks, non-follower constraints can be handled in the pricing problem. By associating the same task of $\mathcal{T}^{B}$ with two different edges, it is guaranteed that any 2-loop-free path will not serve the two edges consecutively (in either direction). For tasks $\mathcal{T}^{B}$, there can only be no or one task per edge. Note further that any properly stronger relaxation, i.e., forbidding task loops up to a longer loop length than two, also guarantees 2-loop-free paths. However, such a relaxation is too restrictive in the sense that it would also exclude paths that are explicitly allowed in the non-follower branch, e.g., a path that contains a single 3-loop. 
In essence, a shortest-path problem where paths are elementary w.r.t. $\mathcal{T}^{E}$ and task-2-loop-free w.r.t. $\mathcal{T}^{B}$ must be solved. In the following, we will skip the 'task-' prefix. Consequently, 2-loop-free tours are indispensable, since the only viable branching scheme (known to us) is based on follower and non-follower constraints resulting in edges having identical tasks.

Let $P$ be any path in $G$. The following attributes are associated with $P$ in a labeling procedure:

$i(P)=$ the end node of path $P$

$\tilde{c}(P)=$ the accumulated reduced cost along $P$

$q(P)=$ the accumulated load along $P$

$\mathcal{T}^{E}(P)=$ the sequence of tasks from $\mathcal{T}^{E}$ in the ordering as serviced by $P$

$\mathcal{T}^{B}(P)=$ the last task form $\mathcal{T}^{B}$ serviced by $P$; if $P$ is a pure deadheading path then $\mathcal{T}^{B}(P)=$.

Note that we just need to keep track of the last task $\mathcal{T}^{B}(P)$ in any dominance algorithm, while for the tasks $\mathcal{T}^{E}(P)$ the sequence, a part of the sequence or a subset of the tasks might be relevant depending on the respective relaxation.

A feasible path $P$ ending at $i=i(P)$ can be extended along an edge either deadheaded or serviced. Any deadheading extension along an edge $e=\{i, j\} \in \delta(i)$ with associated reduced cost $\tilde{c}_{e}$ is feasible. The resulting new path $P^{\prime}$ has the following attributes:

$$
\begin{aligned}
i\left(P^{\prime}\right) & =j \\
\tilde{c}\left(P^{\prime}\right) & =\tilde{c}(P)+\tilde{c}_{e} \\
q\left(P^{\prime}\right) & =q(P) \\
\mathcal{T}^{E}\left(P^{\prime}\right) & =\mathcal{T}^{E}(P) \\
\mathcal{T}^{B}\left(P^{\prime}\right) & =\mathcal{T}^{B}(P)
\end{aligned}
$$

On the other hand, a service extension along an edge $e=\{i, j\} \in \delta_{R}(i)$ with associated reduced cost $\tilde{c}_{e}^{\text {serv }}$ is feasible if $q(P)+q_{e} \leq C$ holds. Moreover, in the ESPPRC case, the task sequences $\mathcal{T}^{E}(P)$ and $\mathcal{T}^{E}(i, j)$ must have no task in common, and $\mathcal{T}^{B}(P) \neq \mathcal{T}^{B}(i, j)$ needs to be fulfilled. If for one or both paths $P$ and $(i, j)$ there is no last task in $\mathcal{T}^{B}$, indicated by ' $\cdot$ ', then the latter condition is always considered true. The resulting new path $P^{\prime}$ has the following attributes:

$$
\begin{aligned}
i\left(P^{\prime}\right) & =j \\
\tilde{c}\left(P^{\prime}\right) & =\tilde{c}(P)+\tilde{c}_{e}^{\text {serv }} \\
q\left(P^{\prime}\right) & =q(P)+q_{e} \\
\mathcal{T}^{E}\left(P^{\prime}\right) & =\left(\mathcal{T}^{E}(P), \mathcal{T}^{E}(i, j)\right) \\
\mathcal{T}^{B}\left(P^{\prime}\right) & =\mathcal{T}^{B}(i, j)
\end{aligned}
$$

In the pure non-elementary case considered by Letchford and Oukil (2009), the attributes $\mathcal{T}^{E}(P)$ and $\mathcal{T}^{B}(P)$ are completely ignored. Then, a path $P$ dominates another path $Q$ if $i(P)=i(Q), \tilde{c}(P) \leq \tilde{c}(Q)$, and $q(P) \leq q(Q)$ holds. The entire labeling procedure is summarized in Algorithm 1 .

Some remarks about Algorithm 1 seem appropriate here:

1. In the non-elementary case, dominance is trivial. The set $\left\{P \in \mathcal{P}_{q}: i(P)=i, q(P)=q\right\}$ for a given combination of $i$ and $q$ contains not more than a single path (sometimes no path). Whenever a new path $P^{\prime}$ is created with load $q$, it replaces the existing one, say $Q$, only if it is cheaper, i.e., $\tilde{c}\left(P^{\prime}\right)<\tilde{c}(Q)$. If paths are stored in arrays (index by node $i(P)$ and load $q(P)$ ) this dominance step needs just constant time $\mathcal{O}(1)$.

2. The use of a Fibonacci heap data structure (see Ahuja et al., 1993) guarantees the worst-case complexity of $\mathcal{O}(|E|+|V| \log |V|)$ of the Dijkstra-like extensions.

3. The final filtering step is necessary, since the algorithm would otherwise output some paths that are not Pareto-optimal. Note that the dominance procedure among all paths ending at the node $d$ requires $\mathcal{O}(C)$ time only because paths $P$ with $i(P)=d$ are already sorted by $q(P)$ (by using the indexing). 


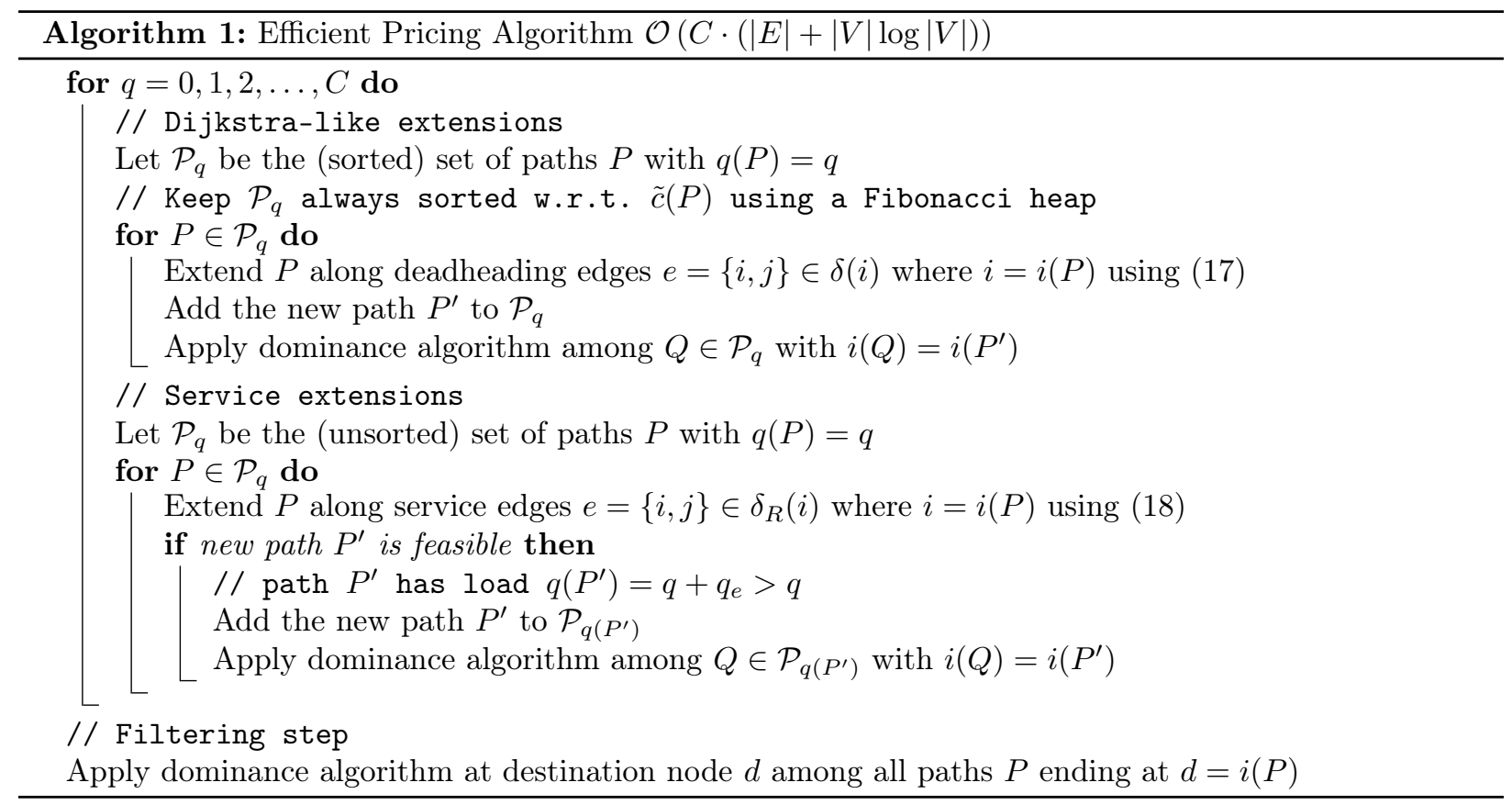

\subsection{2-Loop-free Paths}

The necessary modification for pricing out only 2-loop-free tours is not complicated. In this case, the tasks for non-followers $\mathcal{T}^{B}$ are always a subset of the tasks $\mathcal{T}^{E}$ so that it suffices to be 2-loop-free w.r.t. $\mathcal{T}^{B}$. Therefore, a path $P$ does not record the sequence $\mathcal{T}^{E}(P)$, but the node $i(P)$, the cost $\tilde{c}(P)$, the load $q(P)$, and the last task $\mathcal{T}^{B}(P)$ serviced. A path $P$ dominates a path $Q$ if $i(P)=i(Q), \tilde{c}(P) \leq \tilde{c}(Q)$, $q(P) \leq q(Q)$, and $\mathcal{T}^{B}(P)=\mathcal{T}^{B}(Q)$, i.e., they have the same last task. Moreover, two paths $P_{1}$ and $P_{2}$ with $\mathcal{T}^{B}\left(P_{1}\right) \neq \mathcal{T}^{B}\left(P_{2}\right)$ together dominate any other path $Q$ if $i\left(P_{1}\right)=i\left(P_{2}\right)=i(Q), \tilde{c}\left(P_{1}\right), \tilde{c}\left(P_{2}\right) \leq \tilde{c}(Q)$, $q\left(P_{1}\right), q\left(P_{2}\right) \leq q(Q)$ As a result, there are never more than two relevant paths $P_{1}, P_{2}$ with $i\left(P_{1}\right)=i\left(P_{2}\right)$ and $q\left(P_{1}\right)=q\left(P_{2}\right)$, one with minimum cost and one with second best cost having a different preceding task $\mathcal{T}^{B}\left(P_{1}\right) \neq \mathcal{T}^{B}\left(P_{2}\right)$. Additional algorithmic tricks for implementing 2-loop elimination can be found in Kohl, 1995, Larsen, 1999).

\section{2. $n g$-Route Relaxation}

The $n g$-route relaxation by Baldacci et al. (2011b) has been successfully applied for solving several VRP variants using cut-and-column generation approaches. The relaxation is parameterized and defined by neighborhoods $N_{i}$, one for each node $i \in V$. In the CARP case, $N_{i} \subseteq \mathcal{T}^{E}$, i.e., tasks of service edges define the neighborhoods and herewith the relaxation. The principle of the $n g$-route relaxation is that the full sequence $\mathcal{T}^{E}(P)$ of served tasks associated with a path $P$ is replaced by a subset $\mathcal{T}_{N G}^{E}(P)$ of the tasks $\mathcal{T}^{E}(P)$ in the sequence. It means that some of the tasks from the sequence $\mathcal{T}^{E}(P)$ are disregarded and also the ordering of the tasks is disregarded.

The subset $\mathcal{T}_{N G}^{E}(P) \subseteq \mathcal{T}^{E}$ is defined recursively with the extension of a path $P$ ending at node $i=i(P)$ along an edge $e=\{i, j\} \in \delta(i)$. Any deadheading extension is allowed, and the new task set for the resulting path $P^{\prime}=(P, e, j)$ is

$$
\mathcal{T}_{N G}^{E}\left(P^{\prime}\right)=\mathcal{T}_{N G}^{E}(P) \cap N_{j}
$$

In contrast, the extension along the service edge is considered feasible w.r.t. $\left(N_{i}\right)_{i \in V}$ if and only if

$$
\mathcal{T}_{N G}^{E}(P) \cap\left\{\mathcal{T}^{E}(i, j)\right\}=\emptyset
$$


and, in this case, the new path $P^{\prime}$ has the task subset

$$
\mathcal{T}_{N G}^{E}\left(P^{\prime}\right)=\left(\mathcal{T}_{N G}^{E}(P) \cup\left\{\mathcal{T}^{E}(i, j)\right\}\right) \cap N_{j},
$$

where $\left\{\mathcal{T}^{E}(i, j)\right\}$ denotes the set of tasks in the service sequence $(i, j)$.

The interpretation of this $n g$-route relaxation is that the neighborhoods $N_{i}$ work as filters: Any task $t \in \mathcal{T}^{E}$ serviced along a path $P$ is disregarded whenever $t \notin N_{i}$ for a node $i$ that is visited after that service. Hence, a repeated service becomes possible then.

Dominance between two paths must consider the subset of tasks. A path $P$ dominates another path $Q$ if $i(P)=i(Q), \tilde{c}(P) \leq \tilde{c}(Q), q(P) \leq q(Q)$, and $\mathcal{T}_{N G}^{E}(P) \subseteq \mathcal{T}_{N G}^{E}(Q)$ holds. It can therefore happen that there exist $\mathcal{O}\left(2^{\left|N_{i}\right|}\right)$ different undominated paths $P$ at a node $i(P)$ with identical load $q(P)=q$ for $q \in\{0,1,2, \ldots, C\}$ given.

Obviously, setting all neighborhoods as large as possible, i.e., $N_{i}=\mathcal{T}^{E}$, solves the elementary case, ESPPRC, where no loops w.r.t. to any task are allowed. In the general case, however, an $n g$-route relaxation does not ensure that every feasible path does not contain a 2-loop w.r.t. $\mathcal{T}^{B}$. Therefore, the 2-loop freeness w.r.t. $\mathcal{T}^{B}$ has to be guaranteed additionally. Combining an $n g$-route relaxation w.r.t. $\mathcal{T}^{E}$ and 2-loop-free routes w.r.t. $\mathcal{T}^{B}$ is straightforward using both types of associated attributes. The number of different undominated paths $P$ at a node $i(P)$ with identical load $q(P)=q$ can now grow by a factor of two, to $\mathcal{O}\left(2^{1+\left|N_{i}\right|}\right)$.

\subsection{Partial Elementary}

The concept of partial elementarity was presented by Desaulniers et al. (2008) and applied to the VRP with time windows (VRPTW). Partial elementarity is a special case of an $n g$-route relaxation where all neighborhood sets $N_{i}=N$ are identical for all nodes $i \in V$. Thus, elementarity w.r.t. the subset $N \subset \mathcal{T}^{E}$ must be ensured.

The same attribute updates and dominance rules as for $n g$-route relaxation are applied. Again 2-loop freeness w.r.t. $\mathcal{T}^{B}$ is not fulfilled automatically, therefore, the partial elementarity relaxation w.r.t. $\mathcal{T}^{E}$ and 2-loop-free routes w.r.t. $\mathcal{T}^{B}$ have to be combined. This increases the maximum number of different undominated paths $P$ at the same node and with identical load to $\mathcal{O}\left(2^{1+|N|}\right)$.

\section{4. k-Loop-free Paths}

It is known that solving an SPPRC with $k$-loop elimination is a good compromise between solving ESPPRC and SPPRC. Note that a path is $k$-loop-free if it does not contain a task loop of length $k$ or smaller, e.g., for $k=3$ no 3-loops and no 2-loops. A general labeling algorithm for $k$-loop-free SPPRC was presented by Irnich and Villeneuve (2006). At the time of its invention, it proved to be highly successful for computing optimal solutions to some knowingly hard VRPTW instances.

In (Bode and Irnich, 2012), computational results for solving the linear relaxation of the columngeneration master program with $k$-loop-free pricing were presented. Due to the incompatibility of nonfollower branching with simple $k$-loop elimination for $k \geq 3$, however, the algorithm by Bode and Irnich (2012) did not provide results for branch-and-price.

\section{5. ( $(k, 2)$-Loop-free Paths}

This section contains new theoretical results for labeling procedures that simultaneously consider two sets of tasks for which loop freeness must be guaranteed. In our CARP application, paths are desired to be $k$-loop-free w.r.t. tasks $\mathcal{T}^{E}$, where we would like $k>2$ to be as large as possible (of course there is the trade-off between strength of the relaxation and effort for pricing), and need to be exactly 2-loop-free w.r.t. the tasks $\mathcal{T}^{B}$. Generalizing, we will derive results for a combined $\left(k_{1}, k_{2}\right)$-loop elimination for the tasks sets $\mathcal{T}^{1}$ and $\mathcal{T}^{2}$. For simplicity, we abbreviate paths feasible w.r.t. both tasks sets $\mathcal{T}^{1}$ and $\mathcal{T}^{2}$ as $\left(k_{1}, k_{2}\right)$-loop-free paths.

It is rather simple to define attribute updates and extension rules for $\left(k_{1}, k_{2}\right)$-loop elimination. The crucial part for an effective labeling algorithm is however the definition of a dominance relation. Straightforward 
approaches define dominance only between paths taking the last $k_{1}-1$ tasks of $\mathcal{T}^{1}$ and $k_{2}-1$ tasks of $\mathcal{T}^{2}$ into account. This is rather easy, but turns out to be ineffective due a possible number of $\mathcal{O}\left(\left|\mathcal{T}^{1}\right|^{k_{1}-1} \cdot\left|\mathcal{T}^{2}\right|^{k_{2}-1}\right)$ labels at the same node and with identical load; see also (Irnich and Villeneuve, 2006) where this point is discussed for node- $k$-cycle elimination. Therefore, the decisive point is the development of effective dominance rules guaranteeing a small number of labels.

Such an effective dominance rule, based on the one for simple $k$-cycle elimination proposed by Irnich and Villeneuve (2006), does not only compare pairs of paths. Instead, several paths together may be needed to dominate another path. In the following, we will distinguish between paths and labels. The paths are represented by labels, but labels may contain additional attributes needed to efficiently test for domination. Moreover, paths can mutually dominate each other, while we will make sure that dominance is uni-directional among labels. This can be achieved using a unique identifier (an ID) for each label, which breaks ties whenever two label with identical resources are compared (in the CARP, the resources are reduced costs and load; for a more detailed discussion of that point see (Irnich and Villeneuve, 2006, p. 393f)).

The dominance principle says that labels $L_{1}, \ldots, L_{s}(s \geq 1)$ representing paths $P_{1}, \ldots, P_{s}$ dominate a label $L$ representing path $P$ if

1. $P_{1}, \ldots, P_{s}$ and $P$ share the same end node denoted by $i\left(P_{1}\right)=\cdots=i\left(P_{s}\right)=i(P)$.

2. Every feasible completion $Q$ of $P$ to the sink node, i.e., $(P, Q)$ is a feasible path, must also result in a feasible path $\left(P_{j}, Q\right)$ for at least one path $P_{j}, j \in\{1, \ldots, s\}$.

3. The cost of $\left(P_{j}, Q\right)$ must not exceed the cost of $(P, Q)$ for all $j \in\{1, \ldots, s\}$.

As a consequence, the label $L$ does not need to be considered in a labeling algorithm because it can never produce a better feasible extension to the destination node than possible with at least one extension of the labels $L_{1}, \ldots, L_{s}$. It is however crucial that the labels $L_{1}, \ldots, L_{s}$ are kept.

The second condition (2.) is typically replaced by a (sufficient) condition that is easier to check, involving resource consumptions and task loops. In fact, all paths $P_{1}, \ldots, P_{s}$ must have not larger loads and reduced costs than $P$, i.e.,

$$
q\left(P_{1}\right), \ldots, q\left(P_{s}\right) \leq q(P) \quad \text { and } \quad \tilde{c}\left(P_{1}\right), \ldots, \tilde{c}\left(P_{s}\right) \leq \tilde{c}(P),
$$

while feasibility regarding tasks loops is not checked via resources.

The fundamental idea for $\left(k_{1}, k_{2}\right)$-loop elimination is to efficiently encode the set of possible extensions of a path. For this purpose, let $\mathcal{E}(P)$ denote the set of loop-free extensions of the path $P$. $\mathcal{E}(P)$ solely considers task loops and not resource consumptions. The second condition above is fulfilled for $P_{1}, \ldots, P_{s}$ and $P$ if 19 and

$$
\bigcup_{i=1}^{s} \mathcal{E}\left(P_{i}\right) \supseteq \mathcal{E}(P)
$$

holds. We will now describe how to encode this condition in order to handle two sets of tasks efficiently.

Encoding the Possible Extensions by Self-Hole Sets. There are two sets of tasks $\mathcal{T}^{1}$ and $\mathcal{T}^{2}$ for which loop freeness has to be ensured. Let $\mathcal{S}$ be the set of all $\left(k_{1}, k_{2}\right)$-loop-free paths, i.e., $k_{1}$-loop-free w.r.t. tasks in $\mathcal{T}^{1}$ and $k_{2}$-loop-free with respect to tasks in $\mathcal{T}^{2}$. Let $P, Q \in \mathcal{S}$ be two feasible paths. Then, the concatenation $(P, Q)$ is also a path in $\mathcal{S}$ if and only if both $\left(\mathcal{T}^{1}(P), \mathcal{T}^{1}(Q)\right)$ is $k_{1}$-loop-free and $\left(\mathcal{T}^{2}(P), \mathcal{T}^{2}(Q)\right)$ is $k_{2}$-loopfree. This condition holds if

$$
\left(\mathcal{T}^{1}(P), \mathcal{T}^{1}(Q)\right)=\left(\ldots, t_{k_{1}-1}^{1}, \ldots, t_{2}^{1}, t_{1}^{1}, s_{1}^{1}, s_{2}^{1}, \ldots, s_{k_{1}-1}^{1}, \ldots\right) \text { with } t_{p}^{1} \neq s_{q}^{1} \text { for all } p+q \leq k_{1}
$$

and

$$
\left(\mathcal{T}^{2}(P), \mathcal{T}^{2}(Q)\right)=\left(\ldots, t_{k_{2}-1}^{2}, \ldots, t_{2}^{2}, t_{1}^{2}, s_{1}^{2}, s_{2}^{2}, \ldots, s_{k_{2}-1}^{2}, \ldots\right) \text { with } t_{p}^{2} \neq s_{q}^{2} \text { for all } p+q \leq k_{2} .
$$

The relevant entries of $\mathcal{T}^{1}(Q)$ and $\mathcal{T}^{2}(Q)$ are the first $k_{1}-1$ and $k_{2}-1$ entries, and we denote these by $\mathcal{T}_{k_{1}}^{1}(Q)$ and $\mathcal{T}_{k_{2}}^{2}(Q)$, respectively. Both sequences $\mathcal{T}_{k_{1}}^{1}(Q)$ and $\mathcal{T}_{k_{2}}^{2}(Q)$ always contain exactly $k_{1}-1$ and $k_{2}-1$ elements, respectively, where missing tasks are represented by a ' $'$ '. 
We are able to express the above condition as

$$
\mathcal{T}_{k_{1}}^{1}(Q) \neq\left(\cdot, \ldots, \cdot, t_{p, i}^{1}, \cdot, \ldots, \cdot\right) \text { for all } p \text { with } 1 \leq p+i \leq k_{1}
$$

and

$$
\mathcal{T}_{k_{2}}^{2}(Q) \neq\left(\cdot, \ldots, \cdot t_{p, i}^{2}, \cdot, \ldots, \cdot\right) \text { for all } p \text { with } 1 \leq p+i \leq k_{2},
$$

where $i$ refers to the $i$ th position in the right-hand-side vector, and $t_{p, i}^{1}$ and $t_{p, i}^{2}$ have the value $t_{p}^{1}$ and $t_{p}^{2}$, respectively. The last $k_{1}-1$ entries of $\mathcal{T}^{1}(P)$, i.e., $t_{p}^{1}$ with $p \in\left\{1, \ldots, k_{1}\right\}$, and the last $k_{2}-1$ entries of $\mathcal{T}^{2}(P)$, i.e., $t_{p}^{2}$ with $p \in\left\{1, \ldots, k_{2}\right\}$ have to be compared with $\mathcal{T}_{k_{1}}^{1}(Q)$ and $\mathcal{T}_{k_{2}}^{2}(Q)$, respectively. It follows that any extension $Q$ of path $P$ is infeasible if $\mathcal{T}_{k_{1}}^{1}(Q)$ or $\mathcal{T}_{k_{2}}^{2}(Q)$ matches with the respective tuple (still '.' refers to an unspecified entry).

These infeasible extensions can be represented by set forms, a concept introduced first in (Irnich and Villeneuve, 2006): The tuples on the right hand side of the above inequality are in fact set forms. The finite union of such set forms defines the self-hole set $H(P)$.

Example 1. For (4,2)-loop elimination in the CARP context, i.e., $k_{1}=4, k_{2}=2, \mathcal{T}_{k_{1}}^{1}=\mathcal{T}_{4}^{E}$ and $\mathcal{T}_{k_{2}}^{2}=\mathcal{T}_{2}^{B}$, let path $P$ have $\mathcal{T}_{4}^{E}(P)=(a, b, c)$ and $\mathcal{T}_{2}^{B}(P)=(\alpha)$. It means that the last three required edges serviced were the edges $a, b$, and $c$. In addition, we are in a branch of the branch-and-price search tree where a non-follower constrained is active, e.g., say for the edges $c$ and $d$, imposing that they have the new identical task $\alpha$ assigned in order to prevent $c$ and d being serviced consecutively.

Then, any extension $Q$ produces a feasible path w.r.t. loop elimination if

$$
\left(\mathcal{T}_{4}^{E}(Q), \mathcal{T}_{2}^{B}(Q)\right) \neq(\cdot, \cdot, \cdot)(\alpha),(a, \cdot, \cdot)(\cdot),(b, \cdot, \cdot)(\cdot),(\cdot, b, \cdot)(\cdot),(c, \cdot, \cdot)(\cdot),(\cdot, c, \cdot)(\cdot),(\cdot, \cdot, c)(\cdot) .
$$

Equivalently, the self-hole set $H(P)$ of $P$ is

$$
H(P)=(\cdot, \cdot, \cdot)(\alpha) \cup(a, \cdot, \cdot)(\cdot) \cup(b, \cdot, \cdot)(\cdot) \cup(\cdot, b, \cdot)(\cdot) \cup(c, \cdot, \cdot)(\cdot) \cup(\cdot, c, \cdot)(\cdot) \cup(\cdot, \cdot, c)(\cdot),
$$

where each set form encodes the set of task sequences matching the respective pattern.

For example, if a path $Q_{1}$ produces the task sequence $\mathcal{T}_{4}^{E}\left(Q_{1}\right)=(d, a, b)$ and $\mathcal{T}_{2}^{B}\left(Q_{1}\right)=(\beta)$ then there is no match with $H(P)$, and the extension $\left(P, Q_{1}\right)$ is feasible w.r.t. loop elimination. In contrast, for a path $Q_{2}$ with task sequence $\mathcal{T}^{E}\left(Q_{1}\right)=(d, e, c)$ there is a match so that $(P, Q)$ is infeasible.

The representation of $H(P)$ as the union of set forms is quadratic in $k_{1}$ and $k_{2}$, i.e., up to $\frac{k_{1}\left(k_{1}-1\right)}{2}+$ $\frac{k_{2}\left(k_{2}-1\right)}{2}$ different set forms are necessary to describe all infeasible extensions of path $P$.

Now we consider a dominance situation where (19) and (20) are fulfilled for dominating paths $P_{1}, \ldots, P_{s}$ and a dominated path $P$. By de Morgan's law, we get

$$
\bigcup_{i=1}^{p} \mathcal{E}\left(P_{i}\right) \supseteq \mathcal{E}(P) \Longleftrightarrow \bigcap_{i=1}^{p} H\left(P_{i}\right) \subseteq H(P)
$$

so that the condition 20 for loop-free extensions can be equivalently stated with the help of self-hole sets. The point is now that any intersection of the self-hole sets, resulting on the right hand side, can be calculated and represented as a union of set forms again.

Example 2. Continuing Example 1, let $P^{\prime}$ be another path with $\mathcal{T}_{4}^{E}\left(P^{\prime}\right)=(\cdot, a, d)$ (just two edges serviced along $\left.P^{\prime}\right)$ and $\mathcal{T}_{2}^{B}\left(P^{\prime}\right)=(\beta)$. The self-hole set of $P^{\prime}$ is

$$
H\left(P^{\prime}\right)=(\cdot, \cdot, \cdot)(\beta) \cup(a, \cdot, \cdot)(\cdot) \cup(\cdot, a, \cdot)(\cdot) \cup(d, \cdot, \cdot)(\cdot) \cup(\cdot, d, \cdot)(\cdot) \cup(\cdot, \cdot, d)(\cdot)
$$

Then, the intersection is of self-hole sets is

$$
\begin{aligned}
H(P) \cap H\left(P^{\prime}\right)= & (a, \cdot, \cdot)(\alpha) \cup(\cdot, a, \cdot)(\alpha) \cup(d, \cdot, \cdot)(\alpha) \cup(\cdot, d, \cdot)(\alpha) \cup(\cdot, \cdot, d)(\alpha) \cup \\
& (a, \cdot, \cdot)(\beta) \cup(b, \cdot, \cdot)(\beta) \cup(\cdot, b, \cdot)(\beta) \cup(c, \cdot, \cdot)(\beta) \cup(\cdot, c, \cdot)(\beta) \cup(\cdot, \cdot, c)(\beta) \cup \\
& (a, d, \cdot)(\cdot) \cup(a, \cdot, d)(\cdot) \cup(b, a, \cdot)(\cdot) \cup(b, d, \cdot)(\cdot) \cup(b, \cdot, d)(\cdot) \cup(a, b, \cdot)(\cdot) \cup(d, b, \cdot)(\cdot) \cup \\
& (\cdot, b, d)(\cdot) \cup(c, a, \cdot)(\cdot) \cup(c, d, \cdot)(\cdot) \cup(c, \cdot, d)(\cdot) \cup(a, c, \cdot)(\cdot) \cup(d, c, \cdot)(\cdot) \cup(\cdot, c, d)(\cdot) \cup \\
& (a, \cdot, c)(\cdot) \cup(\cdot, a, c)(\cdot) \cup(d, \cdot, c)(\cdot) \cup(\cdot, d, c)(\cdot)
\end{aligned}
$$


The computation of the intersection of two unions of set forms, as in the above example, requires two algorithmic components: First, set forms need to be tested for inclusion. For example, $(a, \cdot, b, e)(\alpha)$ is included in $(\cdot, \cdot, b, \cdot)(\alpha)$, while $(a, e, b)(\cdot)$ is not included in $(a, \cdot, c)(\cdot)$. It can be shown similarly as for simple $k$-loop elimination, that this test requires only $\mathcal{O}\left(k_{1}+k_{2}\right)$ time and space (Irnich and Villeneuve, 2006, p. 398).

Second, proper intersections of set forms need to be computed. For two set forms $s$ and $t$, the intersection $s \cap t$ is either empty, whenever different entries are specified at the same position. For example, $s=(a, b, \cdot)(\alpha)$ and $t=(a, c, b)(\alpha)$ result in $s \cap t=\emptyset$. Moreover, by definition, the intersection is empty if an infeasible loop is created, e.g., the intersection of $(a, b, \cdot)(\alpha)$ and $(\cdot, b, a)(\cdot)$ is empty, while $(a, b, \cdot)(\alpha, \cdot)$ and $(\cdot, b, d)(\cdot, \cdot)$ have non-empty intersection $(a, b, d)(\alpha, \cdot)$. Here again, the computation including loop detection requires only $\mathcal{O}\left(k_{1}+k_{2}\right)$ amortized time and space. As a result, the computation of the intersection of two self-hole sets, say with $p$ and $q$ set forms each, requires $\mathcal{O}\left(\left(k_{1}+k_{2}\right) p q\right)$ amortized time and space; see (Irnich and Villeneuve, 2006, p. 398) for details.

In order to know the overall time complexity, it is important to quantify the maximum number of elements present in an intersection of two collections of set forms. The next paragraphs will give an answer.

Upper Bound on the Number of Set Forms in an Intersection of Self-Hole Sets. For simple $k$-loop elimination, any collection of set forms resulting from the intersection of self-hole sets does not contain more than $(k-1) !^{2}$ different set forms. This result is stated in (Irnich and Villeneuve, 2006, p. 399) for node- $k$-cycle elimination. Notice that in node- $k$-cycle elimination all paths ending at the same node also share an identical last task (corresponding to that node), which therefore can be omitted. Task- $k$-loop elimination ensures that there are at least $k-1$ other tasks before a task is repeated. Therefore, in both cases, recording only $k-1$ elements is sufficient to encode all relevant dominance information, which results in the stated complexity.

The result for combined $\left(k_{1}, k_{2}\right)$-loop elimination in SPPRC is the following:

Theorem 1. For combined $\left(k_{1}, k_{2}\right)$-loop elimination, the maximum number of different set forms needed to represent any intersection of self-hole sets $H\left(P_{1}\right) \cap H\left(P_{2}\right) \cap \cdots \cap H\left(P_{l}\right)$ of any set of $l$ paths is $\left(k_{1}-1\right) !^{2}$. $\left(k_{2}-1\right) !^{2} \cdot\left(\begin{array}{c}\left(k_{1}-1\right)+\left(k_{2}-1\right) \\ k_{1}-1\end{array}\right)$. This bound is tight.

A proof of this and all other theorems is included in the Appendix. The following example shows how to construct instances where the bound is indeed tight.

Example 3. Consider a combined (3,2)-loop elimination. Moreover, let $P_{1}, P_{2}$, and $P_{3}$ be three paths with no tasks in common. Thus,

$$
\begin{aligned}
& H\left(P_{1}\right)=(\cdot, \cdot)(\alpha) \cup(a, \cdot)(\cdot) \cup(b, \cdot)(\cdot) \cup(\cdot, b)(\cdot) \\
& H\left(P_{2}\right)=(\cdot, \cdot)(\beta) \cup(c, \cdot)(\cdot) \cup(d, \cdot)(\cdot) \cup(\cdot, d)(\cdot) \\
& H\left(P_{3}\right)=(\cdot, \cdot)(\gamma) \cup(e, \cdot)(\cdot) \cup(f, \cdot)(\cdot) \cup(\cdot, f)(\cdot)
\end{aligned}
$$

giving rise to

$$
\begin{aligned}
H\left(P_{1}\right) \cap H\left(P_{2}\right) \cap H\left(P_{3}\right)= & (\gamma)(a, d) \cup(\gamma)(b, d) \cup(\gamma)(c, b) \cup(\gamma)(d, b) \cup(\alpha)(c, f) \cup(\alpha)(d, f) \\
& \cup(\alpha)(e, d) \cup(\alpha)(f, d) \cup(\beta)(a, f) \cup(\beta)(b, f) \cup(\beta)(e, b) \cup(\beta)(f, b) .
\end{aligned}
$$

These are twelve set forms which is the maximum number $\left(k_{1}-1\right) !^{2} \cdot\left(k_{2}-1\right) !^{2} \cdot\left(\begin{array}{c}k_{1}-1+k_{2}-1 \\ k_{1}-1\end{array}\right)=(3-1) !^{2}$. $(2-1) !^{2} \cdot\left(\begin{array}{c}(3-1)+(2-1) \\ 3-1\end{array}\right)=4 \cdot 1 \cdot 3=12$.

Upper Bound on the Number of Paths with Identical Resource Vectors. The paragraph above presented results on the number of set forms in an intersection of an arbitrary number of paths. The question considered in this paragraph is about the maximum number of paths $P$ with identical resource vectors (for the CARP, with identical load $q(P)$, the costs $\tilde{c}(P)$ may differ $)$. Let a collection of $s$ paths $P_{1}, \ldots, P_{s}$ with identical resource vectors ending at a node $i=i\left(P_{1}\right)=\cdots=i\left(P_{s}\right)$ be given. The corresponding labels can be sorted in a unique way using the IDs of the labels so that the following ordering of the paths is given:

$$
P_{1} \prec_{\text {dom }} P_{2} \prec_{d o m} \ldots \prec_{d o m} P_{s}
$$


meaning that, e.g., $P_{s}$ is dominated by all other paths $P_{1}, P_{2}, \ldots, P_{s-1}$. It follows for the intersections of the self-hole sets of the dominating labels $\left(P_{1}\right.$ dominates $P_{2}, P_{1}$ and $P_{2}$ dominate $P_{3}$ etc.) that

$$
I_{1}:=H\left(P_{1}\right) \quad \supseteq \quad I_{2}:=H\left(P_{1}\right) \cap H\left(P_{2}\right) \quad \supseteq \quad \ldots \quad \supseteq \quad I_{s}:=\bigcap_{i=1}^{s} H\left(P_{i}\right) .
$$

holds. Irnich and Villeneuve $(2006)$ have shown that a path $P_{t}$ can be discarded if $I_{t}=I_{t-1}$ holds. Therefore, the maximum length of a properly decreasing chain of intersections of self-hole sets is a bound on the maximum number of labels to consider with identical resource vector.

Theorem 2. A collection of $s$ dominating paths $P_{1} \prec_{\text {dom }} P_{2} \prec_{\text {dom }} \ldots \prec_{\text {dom }} P_{s}$ ending at the same node is given. Let the intersections of the corresponding self-hole sets $H\left(P_{1}\right), H\left(P_{2}\right), \ldots, H\left(P_{s}\right)$ form a properly decreasing chain, i.e. $H\left(P_{1}\right) \supsetneq H\left(P_{1}\right) \cap H\left(P_{2}\right) \supsetneq \cdots \supsetneq \cap_{i=1}^{s} H\left(P_{i}\right)$. Then, the length $q$ of the properly decreasing chain is bounded by $\alpha\left(k_{1}, k_{2}\right)=\left[k_{1}+k_{2}-1\right] \cdot\left(k_{1}-1\right) !^{2} \cdot\left(k_{2}-1\right) !^{2} \cdot\left(\begin{array}{c}\left(k_{1}-1\right)+\left(k_{2}-1\right) \\ k_{1}-1\end{array}\right)$.

For the special case of a combined $(k, 2)$-loop elimination, i.e., for $k_{1}=k$ and $k_{2}=2$, the bound is $\alpha(k, 2)=(k+1) \cdot(k-1) !^{2} \cdot k=(k-1) ! \cdot(k+1) !$. In particular, we get the bounds $\alpha(3,2)=2 \cdot 24=48$ and $\alpha(4,2)=6 \cdot 120=720$ for $k=3$ and 4 , respectively.

\subsection{Scaling}

Scaling of instances is a technique often used in approximation algorithms (Vazirani, 2001). Depending on its concrete implementation, scaling can either provide relaxations or restrictions to a problem. Therefore, lower and upper bounds can result.

In the vehicle routing context, scaling of the demand $q_{i}$ was e.g. considered by Fukasawa et al. $(2006)$. They use it as a heuristic, i.e., a restriction of the pricing problem. For a given scaling factor $f$, both the demand and the capacity are modified via $q_{e}^{\prime}=\left\lceil\frac{q_{e}}{f}\right\rceil$ and $C^{\prime}=\left\lceil\frac{C}{f}\right\rceil$. Obviously, this scaling by factor $f$ has the potential to speed up a labeling algorithm by a factor up to $f$ because the main loop in Algorithm 1 has by the factor $f$ less iterations.

On the other hand, scaling with $q_{e}^{\prime}=\left\lfloor\frac{q_{e}}{f}\right\rfloor$ and $C^{\prime}=\left\lfloor\frac{C}{f}\right\rfloor$ constitutes a pricing relaxation. The expected acceleration when solving the scaled instead of the original instance is also by the factor $f$.

\subsection{Hierarchy of Pricing Relaxations}

All presented pricing relaxations form a hierarchy of relaxations beginning with non-elementary pricing as the weakest relaxation and ending with elementary pricing combined with 2-loop elimination as the strongest. This hierarchy is shown in Figure 1. An arc connecting two relaxations indicates that the tail is a stronger formulation than the head. For example, the relaxation with (4,2)-loop-free routes is stronger than with 4-loop-free routes and $(3,2)$-loop-free routes. The relaxations on the right hand side are parameterized with one or several neighborhoods $N$ and $\left(N_{i}\right)_{i \in V}$ so that these boxes represent families of relaxations. Inside each family, relaxations become stronger the larger the subsets $N$ and $N_{i}$ are (comparable only in case of subset inclusions). Moreover, the $n g$-route relaxation is stronger than the relaxation with partial elementarity whenever $N_{i} \supseteq N$ holds for all nodes $i \in V$.

Shaded boxes $(\square$ ) identify those relaxations that are compatible with our complete branching scheme, in particular, compatible with branching on followers and non-followers. On the other hand, framed boxes $(\square)$ represent pricing relaxations applicable only at the root node (or as long as no branching on followers and non-followers occurs).

\section{Acceleration Techniques}

To use acceleration techniques for fast pricing is essential for the effectiveness of the overall branchand-price approach as outlined by numerous researchers. Some ideas proven useful were summarized in (Desaulniers et al. 2002, Irnich and Desaulniers, 2005). In our case, to run the full exact pricing routine can be time consuming particularly for the $(k, 2)$-loop-free relaxation with larger $k$ and the $n g$-route relaxations with larger neighborhoods $\left(N_{i}\right)_{i \in V}$. To countervail slow pricing, we implemented heuristic and exact acceleration techniques described in the following. 


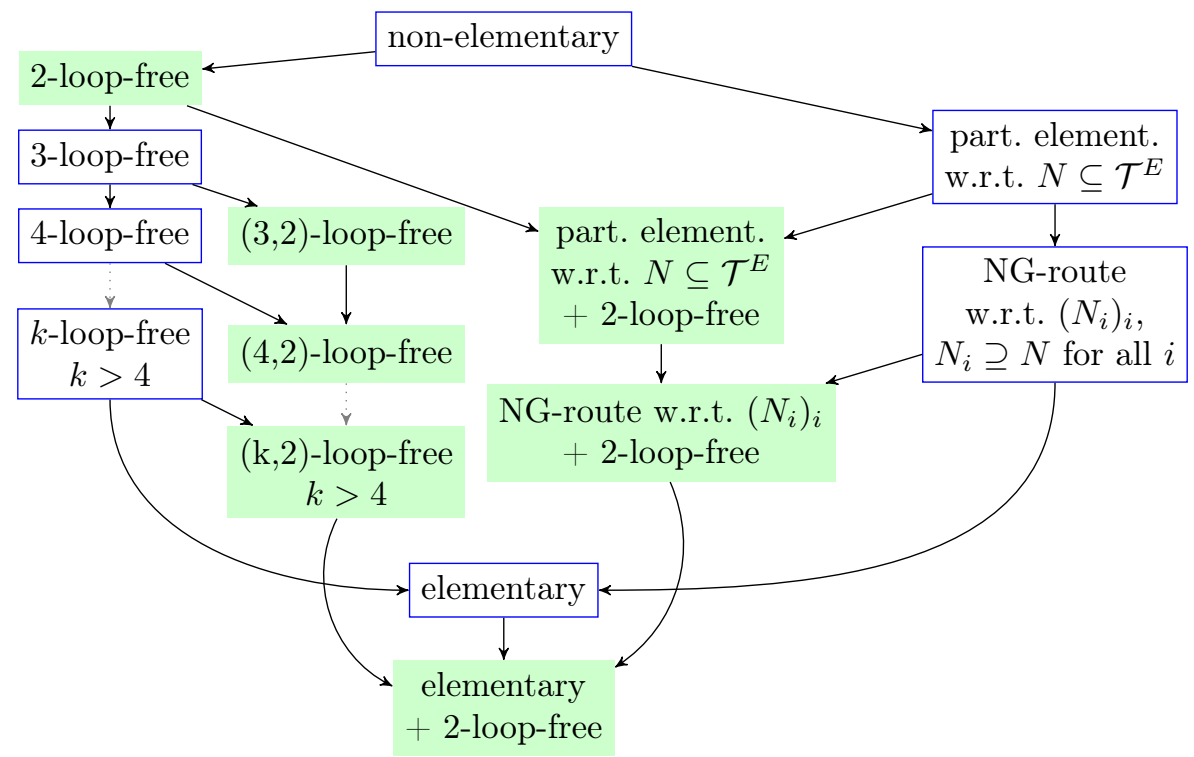

Figure 1: Hierarchy of Pricing Relaxations

\subsection{Pricing Heuristics}

The heuristic labeling algorithms of Letchford and Oukil (2009) for non-elementary pricing can be adapted to 2-loop elimination. They observed that good paths solving the pricing problem often start with deadheading beginning at the depot, followed by a continuous service part, and finish with deadheading back to the depot. Their idea was that a heuristic pricer can restrict itself to assume this structure of the resulting paths.

In order to eliminate 2-loops, a second type of heuristic occurs naturally. Recall that at every node and for every current load, only the best and second best labels with different predecessor tasks have to be stored. Keeping track of the best label only is the second heuristic. It is easy to adapt the same idea in case of $k$-loop and $(k, 2)$-loop elimination. Only if the heuristics fail, the exact pricer is invoked.

\subsection{Bi-Directional Pricing}

As pointed out by Righini and Salani (2006), when solving elementary pricing problems with DP, the number of generated states rapidly increases with the stage and the problem size. They proposed a bidirectional labeling algorithm to partially countervail this effect. It outperforms standard mono-directional pricing algorithms as proven for many node-routing applications. This technique can also be applied for all pricing relaxations discussed in Section 3

Specific to the CARP is that the underlying pricing network is undirected so that forward and backward labeling are identical. Labels for both directions need to be calculated just once. Our critical and only possible resource for bounding is the load. Therefore, we extend paths $P$ only if the current load $q(P)$ is less than or equal to $\lceil C / 2\rceil$. Two generated labels are then combined similar to the procedure join presented in (Righini and Salani, 2006). The main difference is that we merge two paths with common end node, while Righini and Salani (2006) suggest merging over connecting arcs. Two specific implementation details of bidirectional labeling are considered next.

2-Loop-free Paths. A special case occurs when 2-loop-free paths are generated. If the join procedure is implemented in a straightforward fashion, its complexity is $\mathcal{O}\left(|V| C^{2}\right)$ because up to $4(C+1)^{2}$ pairs of paths need to be compared at each node. For the 2-loop-free relaxation, where the number of labels at a node 
does not grow but is constant for increasing values of the load $q$, preliminary tests have shown that the join procedure dominates the run time. Therefore, a more efficient join is needed.

While the standard join finally guarantees the determination of all Pareto-optimal origin-destination paths, we propose a more efficient variant of join with complexity $\mathcal{O}(|V| C)$, which does not guarantee the determination of the complete Pareto frontier. Instead, it is ensured that a least-cost path and all Paretooptimal paths with load not exceeding $C / 2$ are determined. (Generally, many more Pareto-optimal paths are found.) As in the standard case, our join relies on the computation of a set of Pareto-optimal paths $P$ with load $q(P) \leq\lceil C / 2\rceil$ identified with mono-directional labeling. Then it works as follows: For every node and for every value $q=0,1,2, \ldots,\lceil C / 2\rceil$ we determine a best path $P_{1}^{(q)}$ and a second best path $P_{2}^{(q)}$ with $q\left(P_{1}^{(q)}\right), q\left(P_{2}^{(q)}\right) \leq q$, where the last task of the best and the second best path must differ. Then, to generate paths $P$ with load $q(P)>C / 2$, a loop over all values $q=0,1,2, \ldots,\lceil C / 2\rceil$ is performed, and we merge, if feasible, combinations of the paths $P_{i}^{(q)}$ and $P_{j}^{(C-q)}$ for $i, j \in\{1,2\}, i+j \leq 3$ ending at the same node. This requires only $\mathcal{O}(|V| C)$ time and space.

Note that it is non-trivial to transfer the idea to general $(k, 2)$-loop elimination for $k>2$ because there are generally more than two paths with identical load ending at every node. Therefore, the standard join is used here.

ng-Route Relaxation. The half-way test is a component of the join procedure and assures that the same path $P$ with $q(P)>C / 2$ is not generated multiple times. In principle, this happens whenever $P$ can be split differently into $P=(Q, R)$ with $q(Q)>C / 2$. The half-way test proposed by Righini and Salani (2006), in the node-routing context, requires that the split point is chosen as the first node on the path where the critical resource exceeds the bound. In the CARP case, consider a path $Q=\left(Q^{\prime}, e, j\right)$ with last edge $e \in E$ and last node $j$. Then, the half-way test requires that the last edge is serviced so that $q\left(Q^{\prime}\right) \leq C / 2$ and $q(Q)=q\left(Q^{\prime}\right)+q_{e}>C / 2$ holds. As a result, no path $P$ is generated twice.

However, for the CARP and the $n g$-route relaxation, the half-way test is too restrictive. Again, we assume constructing the path $P=(Q, R)$ with $Q=\left(Q^{\prime}, e, j\right)$, i.e., last serviced edge $e \in E_{R}$ and last node $j$. The critical situation is when extending $Q$ to another node $\ell \in V$ and when a task $e^{*} \in \mathcal{T}_{N G}^{E}(Q)$ is not contained in the neighborhood $N_{\ell}$, i.e., $e^{*} \notin N_{\ell}$. Thus, the information that the task $e^{*}$ was serviced along $Q$ is not recorded in a label ending at node $\ell$. Now consider the path $P^{\prime}=\left(Q, e^{\prime}, \ell, e^{\prime}, j\right)$ where the two last extensions are deadheadings along the edge $e^{\prime}=\{j, \ell\} \in E$. The path $P^{\prime}$ dominates path $Q$ w.r.t. resources whenever the deadheading costs $\tilde{c}_{j \ell}=\tilde{c}_{e^{\prime}}$ are zero. Moreover, it may properly dominate w.r.t. $n g$-neighbors because $e^{*} \notin N_{\ell}$. In this case, $Q$ does not exist, but $P^{\prime}$ does not qualify as a forward path in join because its last edge is deadheaded.

In fact, our first implementation contained the (incorrect) half-way test, and cost-minimal paths were missing in very rare occasions. However, it happened that inconsistent bounds were computed in the branchand-price so that this subtle detail became a serious flaw.

Instead of applying the half-way test, we now store for every value $q=0,1, \ldots, C$ a minimum reduced cost joined path and reconstruct on that basis only the Pareto-optimal paths. This is obviously a little less efficient, but the only viable approach known to us.

\subsection{Bounding}

Bounding is intended to reduce the number of states to expand in a DP approach. It has become a key technique for solving the TSP with time windows (Mingozzi et al., 1997, Baldacci et al., 2011c) and variants of the VRP (Baldacci et al. 2009).

In the (E)SPPRC pricing context, for a partial path $P$ at hand, the idea is to calculate a lower bound on the (reduced) cost of any completion to the destination node. If the cost of the path $P$ plus the lower bound exceeds zero, path $P$ can be discarded because it is useless for constructing negative reduced cost routes.

There is a trade-off between the quality of that lower bound and the time needed for its computation. In general, any relaxation of an (E)SPPRC and backward paths generated as solutions to the all-to-destination problem provide feasible lower bounds (for details see, e.g., Baldacci et al. $2011 \mathrm{c}$ ). Note first that in the CARP the network is fully symmetric so that forward and backward labeling is identical. Any relaxation 
solved with mono-directional labeling on the original network so provides lower bounds. The hierarchy of relaxations depicted in Figure 1 offers numerous possibilities for pricing problem relaxations and proper relaxations of these that in combination allow bounding.

For example, 2-loop-free pricing can be used for bounding purposes in combination with any other relaxation compatible with branching. Additionally, $(\ell, 2)$-loop-free tours allow bounding for the $(k, 2)$ loop-free relaxation if $\ell<k$. Even more, in the $n g$-route relaxation with neighborhoods $\left(N_{i}\right)_{i \in V}$, smaller neighborhoods $N_{i}^{\prime} \subset N_{i}$ might be used for bounding. In all cases, we implemented bounding so that the weaker relaxation provides a bounding function $f(i, q)$ defined for every node $i \in V$ and load $q \in$ $\{0,1, \ldots, C\}$. The value $f(i, q)$ is a lower bound on the reduced costs of feasible paths ending at node $i$ with not more than load $q$ on board. When solving the stronger relaxation, any path $P$ with $\tilde{c}(P)+f(i(P), C-$ $q(P))>0$ is identified being useless, and its label can be discarded.

\section{Computational Results}

This section reports computational results of the various pricing relaxations tested when solving the respective linear relaxation and integer formulations of the CARP. The first benchmark set egl was introduced by Eglese and Li (1992) and can be downloaded from http://www.uv.es/ belengue/carp/. This set consists of 24 instances based on the road network of Cumbria. Group e consists of instances with 77 nodes and 98 edges, whereas group $\mathrm{s}$ is larger and has instances with 140 nodes and 190 edges. Each group is further split into four subsets $m \in\{1, \ldots, 4\}$, where the number of required edges increases with $m$. On the lowest level, each subgroup differs in the vehicle capacity, where three different sizes are assumed, indicated by $n \in\{\mathrm{a}, \mathrm{b}, \mathrm{c}\}$. Within each subgroup, the instances a have highest capacity tending to result in less but longer routes, and instances $\mathrm{c}$ have lowest capacity resulting in more but shorter routes. Overall, instance names are coded as follows: egl-lm-n with $l \in\{\mathrm{e}, \mathrm{s}\}, m \in\{1, \ldots, 4\}$, and $n \in\{\mathrm{a}, \mathrm{b}, \mathrm{c}\}$.

The second benchmark set bmcv consisting of 100 instances is obtained from the road network of Flanders, Belgium (Beullens et al. 2003). These instances range from 26 to 97 nodes and 35 to 142 edges, where only a subset of the edges is required. The instances were kindly provided by Muyldermans (2012) and comprise four subsets. The underlying graph for individual instances of subset $\mathrm{C}$ and $\mathrm{E}$ is identical, but the vehicle capacity is 300 for the $\mathrm{C}$ set and 600 for the $\mathrm{E}$ set. The same holds for the subsets of instances named D and $\mathrm{F}$.

\subsection{Computational Setup}

All computations were performed on a standard PC with an Intel@C Core ${ }^{\mathrm{TM}} \mathrm{i} 7-2600$ at $3.4 \mathrm{GHz}$ processor with 16 GB of main memory. The algorithm was coded in C++ (MS-Visual Studio, 2010) and the callable library of CPLEX 12.2 was used to iteratively reoptimize the RMP. A hard time limit of four hours for computation has been set for the column-generation and branch-and-price algorithms.

We tested both $(k, 2)$-loop-free and $n g$-route relaxations with several parameter settings. Within $(k, 2)$ loop-free pricing we varied $k \in\{2,3,4\}$ and the relaxation used for bounding. In detail, for $(3,2)$-loop-free pricing and $n g$-route relaxation we used the 2-loop-free relaxation and for $(4,2)$-loop-free pricing we used both the 2-loop-free and $(3,2)$-loop-free relaxation for bounding. To shorten the notation, we will skip the second entry because it is equal for all $(k, 2)$-loop-free relaxations. Therefore, in the following, $k$-loop is a short-cut for $(k, 2)$-loop-free pricing. In the same spirit we write $4 \mathrm{~b} 2$-loop as a short form of $(4,2)$-loop-free pricing with 2-loop-free bounding.

The choice of neighborhoods $\left(N_{i}\right)_{i \in V}$ has a great impact on the strength of the $n g$-route relaxation and the computational effort needed in every pricing iteration. Because there is an exponential number of possible choices, we decided to focus our analysis to the most influential parameter, which is the maximum size of a neighborhood. Here we ran the algorithms with parameters $n_{n g} \in\{3,4,5,6,7,8,9,10,12,15\}$ meaning that all neighborhood sizes $\left|N_{i}\right|$ do not exceed $n_{n g}$, i.e., for $\left|N_{i}\right| \leq n_{n g}$. To indicate the (maximum) size of the neighborhoods, we write, e.g., $n g 6$ whenever $\left|N_{i}\right| \leq 6$.

There exist several methods of determining the concrete sets $N_{i}$. Desaulniers et al. (2008) proposed an algorithm for partially elementary, i.e., $N_{i}=N$ for all $i \in V$, in which iteratively the linear relaxation 
of the RMP is solved. As long as the neighborhood size $|N|$ is smaller than a predefined maximal size $n_{\max }$ and there exists a task cycle in the solution, this task is added to the neighborhood $N$. Tasks with a large flow on cycles are chosen with priority. On the other hand, Baldacci et al. (2011b) use individual neighborhoods $N_{i}$ for every node $i \in V$. The sets $N_{i}$ are pre-computed by adding a customer $j$ to $N_{i}$ if it is among the $n_{n g}$ nearest nodes to node $i$. We combine these two ideas because we dynamically generate individual neighborhoods $N_{i}$ (a similar idea was presented by R. Roberti in the presentation (Baldacci et al. 2011a)). The procedure is summarized in Algorithm 2

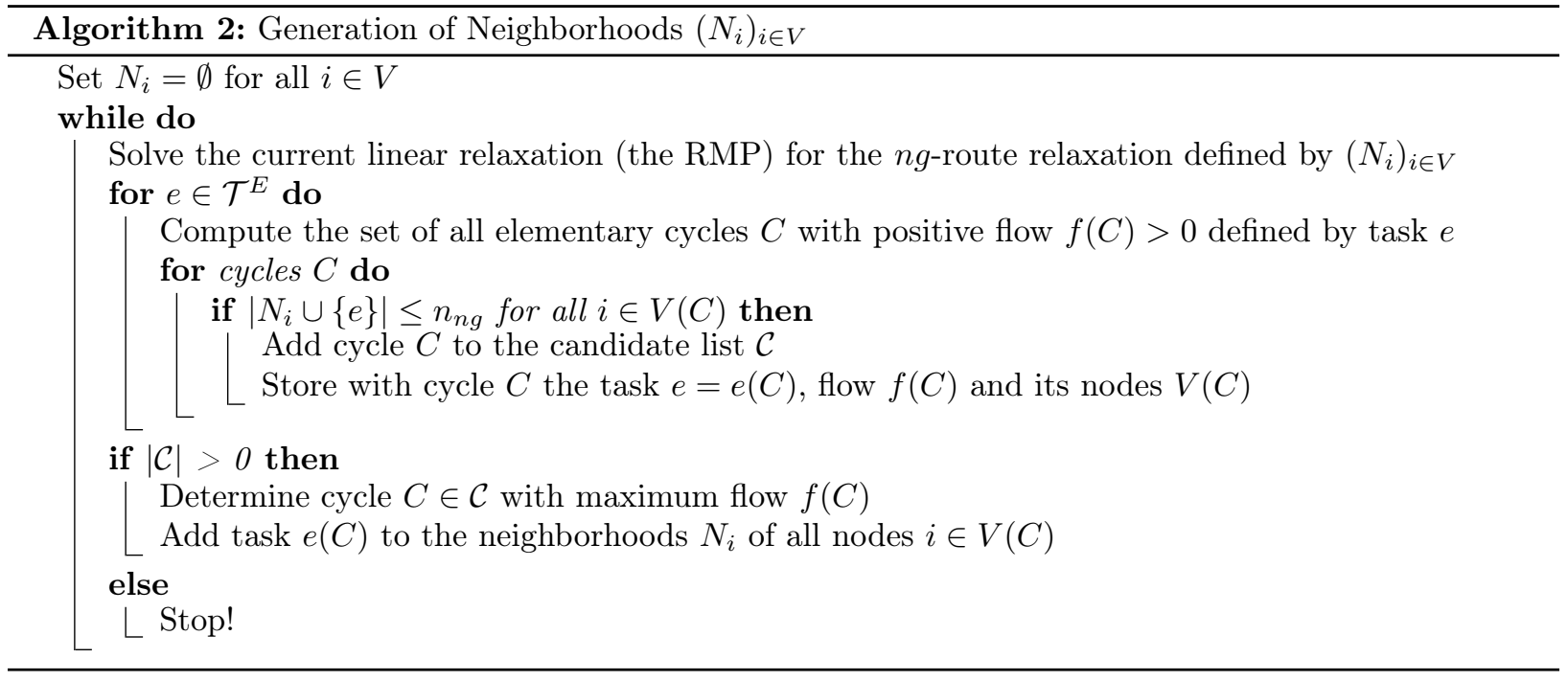

Note that when adding new tasks to a neighborhood $N_{i}$, the resulting relaxation becomes more restrictive so that a formerly feasible route $r$ can become infeasible. Those routes that become infeasible have to be removed from the RMP at the beginning of every main loop of Algorithm 2. Thus, the RMP first gets smaller, while it increases again with every newly generated route.

Finally, bidirectional labeling can be applied in every pricing algorithm. In the following, we indicate bidirectional labeling with the term 'BiDir'.

\subsection{Impact of Acceleration Techniques}

We start with analyzing the impact of the acceleration techniques presented in Section 4 . In order to measure the improvement of bounding and bidirectional pricing for different pricing relaxations, both the root node and the full branch-and-bound tree were solved with no, one, or both techniques active. Computations were performed for all $24 \mathrm{egl}$ instances and the different relaxations. The improvement is then calculated as the ratio of the time for pricing without acceleration and the time with one or both techniques active, respectively, for each instance. For abbreviation, we refer to the these numbers as acceleration factors. For not biasing the acceleration factors, we turned off all heuristic pricing procedures. Figures 2 and 3 show the resulting box-and-whisker diagrams (McGill et al., 1978$)$.

Comparing the results among the $k$-loop-free relaxations, bidirectional pricing has a higher impact the larger $k$ is. For 2-loop, the only acceleration technique is bidirectional pricing, where for the linear relaxation ('Root') the median acceleration factor is 1.4 with $50 \%$ of the data lying in a very small range inside the box. Figure $2 \mathrm{a}$ shows that the acceleration factor is slightly smaller considering the overall branch-and-price tree ('Tree').

This median increases to 3.8 and 5.1 for 3-loop and 4-loop, respectively (see Figures 2b and 2c). For these relaxations, bidirectional pricing has always an impact greater than one, nevertheless the data scatters more. For example, for the instance e4-a solving the root node with bidirectional pricing is about 15 times faster than with the basic 4-loop algorithm, and for the instance s4-c just 2.8 times faster. For indicating 


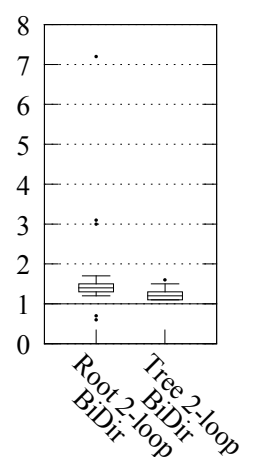

(a) 2-loop

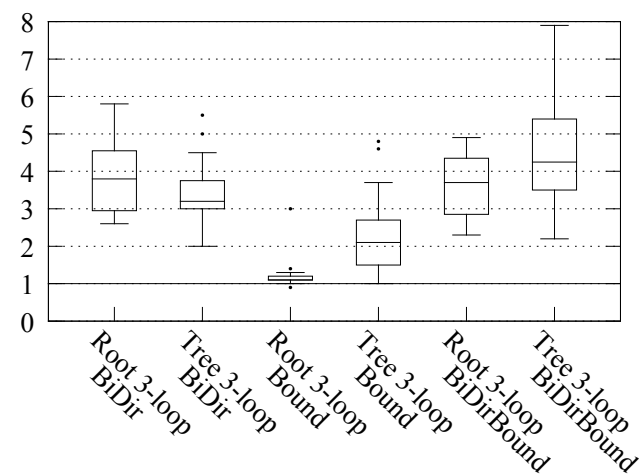

(b) 3-loop

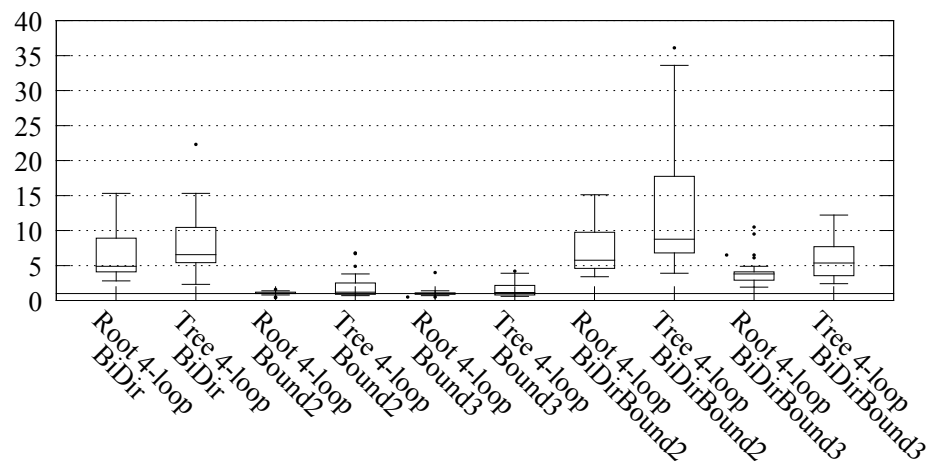

(c) 4-loop

Figure 2: Impact of Bidirectional Pricing and/or Bounding for the $(k, 2)$-loop Relaxations: Box-and-Whisker Diagrams of the Acceleration Factors

the spread of the data, the end of the whiskers show data that lying within the 1.5 interquartile range. Any other data is outliers and they are represented by small dots.

Comparing the results over the full branch-and-bound tree solely using bidirectional pricing, there is an improvement compared to the root node only for 4-loop pricing. However, combined with bounding the positive impact of using acceleration techniques is strengthened. Sometimes a speed up factor of 36 can be reached (instance s2-c in 4b2-loop pricing).

The impact of using bounding alone is very small, in particular for solving the linear relaxation ('Root'). The median within 3-loop pricing is only slightly above 1.0 and the lower whisker is ending at 1.0. There, bounding has always a small but non-negative impact compared to 4-loop pricing. The median for bounding with 2-loop and 3-loop bounding is 1.0 and 0.9, respectively. Hence, bounding alone often results in longer computation times. Considering the whole branch-and-bound tree ('Tree'), the acceleration factors are slightly higher.

Finally, for the relaxation with 4-loop-free routes, the comparison of bounding with the 2-loop and 3-loop shows a clear winner: 2-loop-free bounding is superior to 3-loop-free bounding meaning that slightly better bounds are obtained.

The impact of bidirectional pricing and bounding is, at the root node, very similar for all tested $n g$ route relaxations (see Figures $3 \mathrm{a}$ 3 $3 \mathrm{c}$ ). The median of all acceleration techniques is between approximately 1.5 and 2.0, and the dispersion of the data is not as high as for the $k$-loop relaxations. However, except for solely bounding within $n g 6$, there are instances where solving the root node takes longer than without any acceleration techniques. Similar to $k$-loop, considering the full branch-and-bound tree, the impact of 


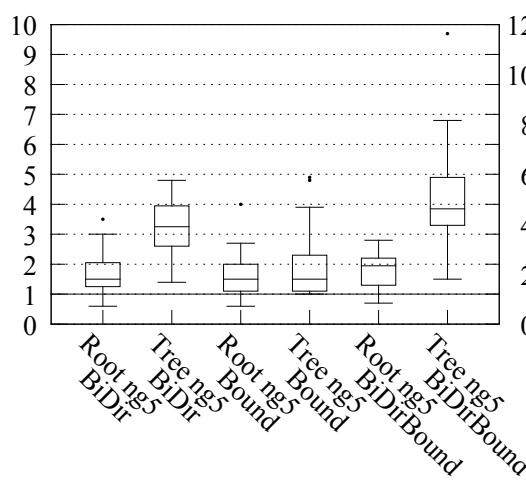

(a) ng5

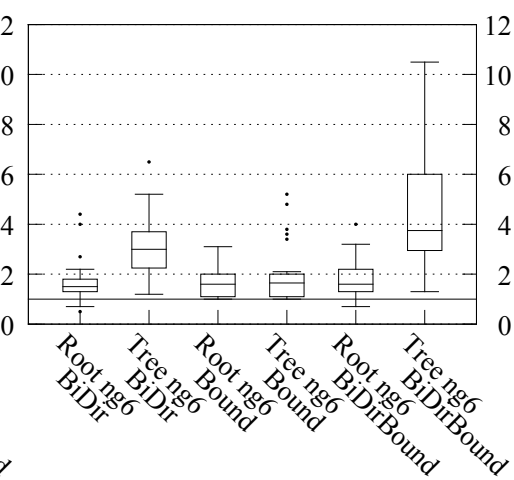

(b) ng6

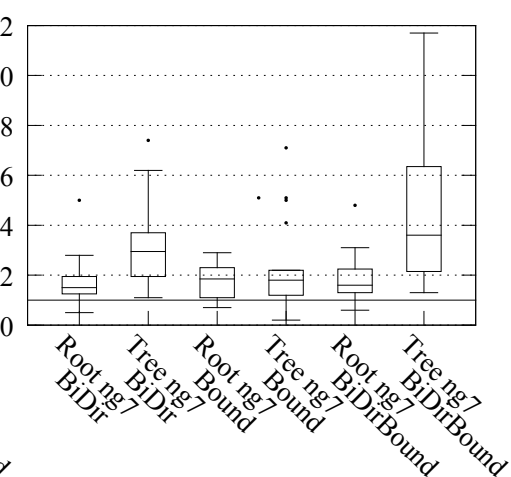

(c) ng7

Figure 3: Impact of Bidirectional Pricing and/or Bounding for the $n g$-route Relaxations: Box-and-Whisker Diagrams of the Acceleration Factors

bounding and/or bidirectional pricing is at least as good as at the root node, but often better. The only exception is bounding within the $n g 7$-route relaxation: The median is approximately the same comparing the root node and the full tree, but there are instances (e.g., e1-b and e2-b) where solving the pricing problem is up to five times slower than the basic $n g 7$-route algorithm. In general, combining all presented acceleration techniques for solving the branch-and-price part gives the best results. Therefore, all following computational results are presented for combined bidirectional pricing with bounding.

\subsection{Linear Relaxation Results}

The focus of the following analysis is on lower bounds obtained with the linear relaxations (at the root node). A comprehensive study for the egl instances and relaxations with $k$-loop elimination was already presented in (Bode and Irnich, 2012). However, no acceleration techniques and no $n g$-route relaxations were considered. Therefore, we will now present lower bounds and computation times for $k$-loop elimination and $n g$-route relaxations with the presented acceleration techniques activated. Table 1 presents aggregated results for the egl instances and Table 2 for the bmcv instances.

Table 1: Aggregated Linear Relaxation Results for egl Instances

\begin{tabular}{r|rrrr|rrr} 
& 2-loop & 3-loop & 4b2-loop & 4b3-loop & $n g 5$ & $n g 6$ & $n g 7$ \\
\hline Minimum gap (\%) & 0.07 & 0.05 & 0.05 & 0.05 & 0.00 & 0.00 & 0.00 \\
Average gap (\%) & 0.84 & 0.74 & 0.68 & 0.68 & 0.61 & 0.59 & 0.58 \\
Maximum gap (\%) & 1.60 & 1.30 & 1.29 & 1.29 & 1.24 & 1.23 & 1.23 \\
Minimum time (s) & 9 & 22 & 21 & 26 & 63 & 67 & 65 \\
Average time (s) & 90 & 233 & 511 & 615 & 1,646 & 2,220 & 2,601 \\
Maximum time (s) & 294 & 837 & 4,151 & 3,660 & 10,507 & 10,016 & 14,306
\end{tabular}

Table 2: Aggregated Linear Relaxation Results for bmcv Instances

\begin{tabular}{r|rrrr|rrr} 
& 2-loop & 3-loop & 4b2-loop & 4b3-loop & $n g 5$ & $n g 6$ & $n g 7$ \\
\hline Minimum gap (\%) & 0.00 & 0.00 & 0.00 & 0.00 & 0.00 & 0.00 & 0.00 \\
Average gap (\%) & 0.55 & 0.54 & 0.52 & 0.52 & 0.48 & 0.48 & 0.48 \\
Maximum gap (\%) & 2.79 & 2.69 & 2.69 & 2.69 & 2.39 & 2.37 & 2.37 \\
Minimum time (s) & 1 & 3 & 2 & 2 & 2 & 2 & 2 \\
Average time (s) & 20 & 317 & 426 & 274 & 1,668 & 2,055 & 2,277 \\
Maximum time (s) & 194 & 22,760 & 14,914 & 12,902 & 14,373 & 14,288 & 14,253
\end{tabular}


In preliminary computational tests we varied $n_{n g}$ more widely including values between $n_{n g}=3$ and $n_{n g}=15$. We tested the relaxations inside the overall branch-and-price algorithm and counted the number of times that a specific relaxation produced the best lower bound at the time limit. It turned out that the relaxations with $n_{n g} \in\{5,6,7\}$ outperformed the others (except for some rare outliers). Hence, we report results for $n g$-route relaxations only for the three parameters $n_{n g} \in\{5,6,7\}$.

Due to the integration of 2-loop-free pricing in $n g$-route relaxations (see Section 3), the lower bounds obtained with any $n g$-route relaxation are always at least as good as the lower bounds with the 2-loop-free relaxation. Therefore, a stronger relaxation results in better lower bounds, i.e., smaller gaps in the best, average, and worst case. Some substantial improvements were observed, e.g., 69 units for the instances egl-e2-c and egl-s1-c. For all relaxations, the minimum gap for the bmcv instances is zero meaning that at the root node the gap is closed. As expected, solving the linear relaxation becomes more time consuming for both increasing values of $k$ and $n_{n g}$. However, bounds alone do not provide a comprehensive assessment because, on the average, solving the root node with $k$-loop relaxation is significantly faster than with an $n g$-route relaxation. Detailed results with lower bound values and computation times for all instances can be found in the Appendix in Tables 68

\subsection{Integer Solution Results}

Next we summarize integer results for the egl and bmcv instances. Given the time limit of four hours $(14,400 \mathrm{~s})$ for solving each instance, we report the number of instances solved to optimality ('Num. opt. sol.'), the number of instances where the respective relaxation produced the best lower bound among all tested relaxations ('Num best $l b$ '), and the remaining gap at the end of the branch-and-price tree (using the best known upper bound $u b$ ). Note that the node-selection rule was best first. Aggregated results are presented in Tables 3 and 4 , while detailed results for individual instances can be found in the Appendix in Tables 911 .

Table 3: Aggregated Integer Results for egl Instances

\begin{tabular}{|c|c|c|c|c|c|c|c|c|}
\hline & 2-loop & 3-loop & 4b2-loop & 4b3-loop & $n g 4$ & $n g 5$ & $n g 6$ & $n g 7$ \\
\hline Num. opt. sol. (all/a/b/c) & $5 / 4 / 1 / 0$ & $6 / 4 / 2 / 0$ & $6 / 4 / 2 / 0$ & $6 / 4 / 2 / 0$ & $4 / 3 / 1 / 0$ & $3 / 2 / 1 / 0$ & $4 / 2 / 2 / 0$ & $3 / 1 / 2 / 0$ \\
\hline Num. best $l b($ all $/ \mathrm{a} / \mathrm{b} / \mathrm{c})$ & $7 / 6 / 1 / 0$ & $6 / 4 / 2 / 0$ & $7 / 4 / 2 / 1$ & $6 / 4 / 2 / 0$ & $6 / 3 / 2 / 1$ & $6 / 3 / 2 / 1$ & $13 / 2 / 5 / 6$ & $13 / 2 / 4 / 7$ \\
\hline Average gap (\%) & 0.69 & 0.62 & 0.57 & 0.58 & 0.48 & 0.43 & 0.44 & 0.43 \\
\hline Maximum gap (\%) & 1.12 & 1.09 & 1.09 & 1.10 & 1.04 & 1.06 & 1.06 & 1.07 \\
\hline
\end{tabular}

Table 4: Aggregated Integer Results for bmcv Instances

\begin{tabular}{|c|c|c|c|c|}
\hline & 2-loop & 3-loop & 4b2-loop & 4b3-loop \\
\hline Num. opt. sol. (all/C/D/E/F) & $75 / 17 / 21 / 15 / 22$ & $75 / 17 / 20 / 16 / 22$ & $76 / 17 / 19 / 19 / 21$ & $76 / 17 / 19 / 19 / 21$ \\
\hline Num. best $l b($ all/C/D/E/F) & $85 / 21 / 23 / 16 / 25$ & $72 / 17 / 19 / 14 / 22$ & $72 / 17 / 18 / 17 / 20$ & $67 / 17 / 16 / 15 / 19$ \\
\hline Average gap (\%) & 0.31 & 0.34 & 0.42 & 0.41 \\
\hline \multirow[t]{2}{*}{ Maximum gap (\%) } & 1.48 & 2.03 & 2.23 & 2.26 \\
\hline & $n g 5$ & $n g 6$ & $n g 7$ & \\
\hline Num. opt. sol. (all/C/D/E/F) & $76 / 18 / 19 / 19 / 20$ & $75 / 18 / 19 / 18 / 20$ & $76 / 18 / 19 / 19 / 20$ & \\
\hline Num. best $l b($ all $/ \mathrm{C} / \mathrm{D} / \mathrm{E} / \mathrm{F})$ & $71 / 21 / 15 / 19 / 16$ & $69 / 22 / 14 / 18 / 15$ & $68 / 20 / 12 / 21 / 15$ & \\
\hline Average gap (\%) & 0.37 & 0.39 & 0.41 & \\
\hline Maximum gap (\%) & 2.20 & 2.26 & 2.26 & \\
\hline
\end{tabular}

For the egl instances, the $k$-loop relaxations are able to find more integer solutions, while for the bmcv the $n g$-route relaxation and the $k$-loop relaxations produce approximately the same number of optima. Whenever the time limit is reached, $n g 6$ and $n g 7$ produce the best lower bounds for the egl instances, and both the average and maximum gap is generally better for $n g$-route relaxations. In contrast, for bmcv instances, the 2-loop relaxation gives the best solutions both on average and with respect to the maximum gap. However, there is the tendency that the 2-loop relaxation can solve problems of groups with higher 
vehicle capacity (i.e. egl-lm-a and bmcv D and F) better $(6,23$, and 25 best lower bounds), while the best $n g$-route relaxation, i.e., $n g 7$, performs worse on these instances (only 2, 12, and 15 best lower bounds). On the other hand, for instances with lower capacity, i.e., egl-lm-c, bmcv C and E, the 2-loop-free relaxations results in 0,21 , and 16 best lower bounds, while $n g 7$ gives 7,20 , and 21 best results. The detailed analysis of those groups of instances and the question why one relaxation performs good on one group and poorly on another is subject of the next section.

\subsection{Performance Analysis by Instance Groups}

There is no single relaxation that solves all types of instances best, i.e., provides the tightest lower bounds in least time. Instead, different groups of instances are best solved with different relaxations. We will identify the groups of instances and the most effective relaxations for each group and we will try to explain the reasons why a relaxation is more or less effective. For that purpose, we will provide different analyses about the time that the components of a branch-and-price require.

Lower Bounds over Time. A first insightful analysis is the evolution of the lower bound values over time for different instances and pricing relaxations. For the egl instances, the principal behavior is mainly affected by the parameter $n \in\{\mathrm{a}, \mathrm{b}, \mathrm{c}\}$ leading to groups egl-lm-a, egl-lm-b and egl-lm-c. A typical example is shown for the three instances egl-e4- $n$ in Figure 4 . These instances have been chosen because none of the relaxations is able to prove optimality within the time limit. Hence, bounds can be compared over the full four hours.

As shown in Figure 4 a for egl-e4-a, the same lower bound values are reached approximately ten times faster with the 2-loop-free relaxation than with any other relaxation. Similarly, for almost all egl-lm-a instances, the 2-loop-free relaxation outperforms all other relaxations. An exception is instance egl-s1-a, were both the 3-loop-free and 4b2-loop-free relaxation prove optimality in less time. Further exceptions are the instances egl-s2-a and egl-s4-a, where the $n g$-route relaxations outrun after approximately half of the available time all $k$-loop-free relaxations. They end up with a bound three units better than the 2-loop-free relaxation. The Appendix provides detailed figures for all egl instances and relaxations and lower bound values over time.

Typical for all egl-lm-b instances is the existence of an intersection point from where on the $n g$-route relaxations become more effective than the $k$-loop-free-relaxations. For example, in Figure 4b, the 2-loopfree relaxation performs better than the $n g 6$ relaxation before that point and less effective afterwards. This intersection point is at approximately 100 seconds for egl-em-b instances and at about 1,000 seconds for egl-s $m$-b instances.

If just a few seconds of computation time are available, the egl-lm-c instances are solved best with an $n g$-route relaxation compared to 3-loop and 4-loop relaxations (2-loop-free is not competitive at all). Moreover, the $n g$-route relaxation delivers sometimes significantly better lower bounds at the end, see Figure $4 \mathrm{c}$ Compared to the egl-lm-a group, the performance order of the relaxations is reversed for group c.

Branching Decisions and Relative Times. To explain this behavior an even more detailed analysis of the algorithms is done for the 2-loop-free and $n g 6$ relaxations. The number of branch-and-bound nodes solved and the type of branching decision taken impacts which and how often a particular algorithmic component is invoked. Therefore, we recorded the number and the type of of branching decisions. Moreover, we kept track of the relative times spent (1) on updating the RMP ('update'), i.e., addition and removal of constraints and columns as well as the modification of the network, (2) for re-optimizing the RMP ('re-opt') using the primal simplex method (on average a little faster than the dual simplex algorithm), (3) for pricing ('pricing'), and (4) for other components ('other'). For egl-e4- $n$, these numbers are depicted in Figure 5.

One can notice in Figure 5 a that for both the 2-loop-free relaxation and the $n g 6$ relaxation, the number of solved branch-and-bound nodes increases from a to c. This results from the fact that due to the choice of demands and capacities, the routes are on average longer in $\mathrm{a}$, become shorter for $\mathrm{b}$, and are shortest for $\mathrm{c}$ instances. Longer routes require longer computation times per pricing iteration leading to longer computing times per node. 


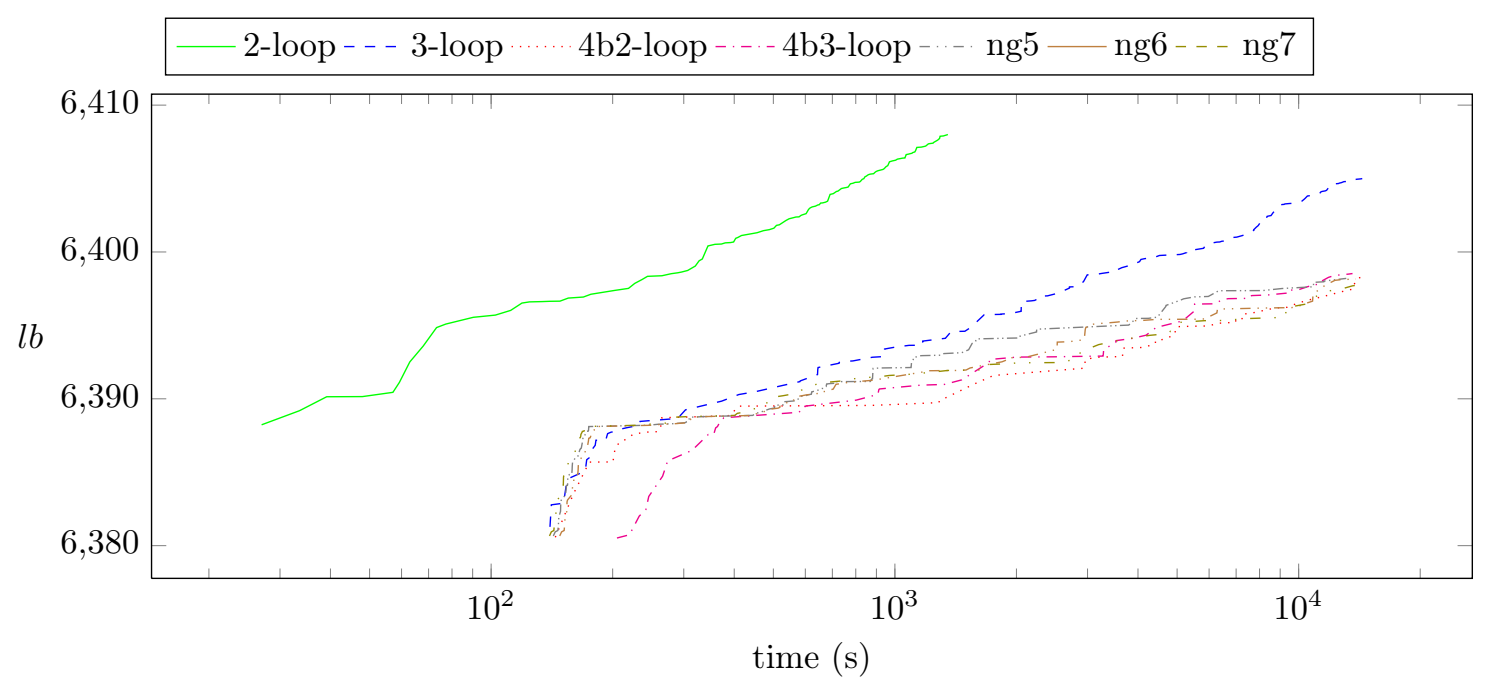

(a) egl-e4-a

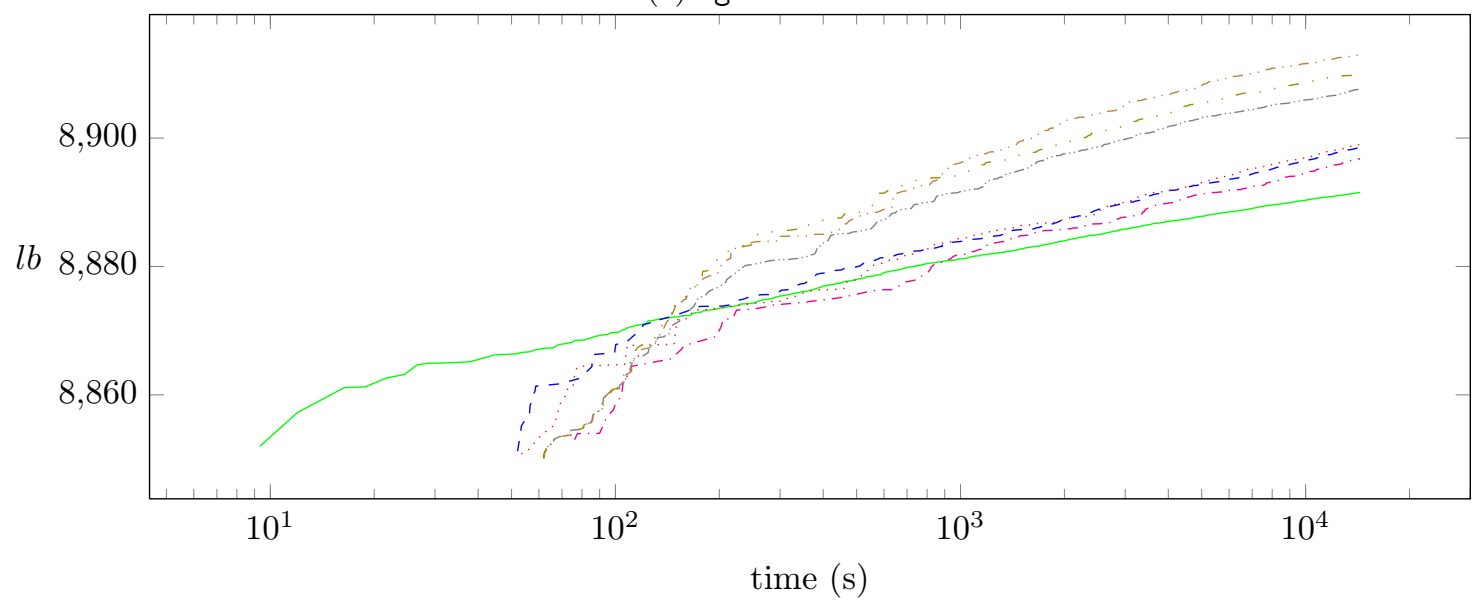

(b) egl-e4-b

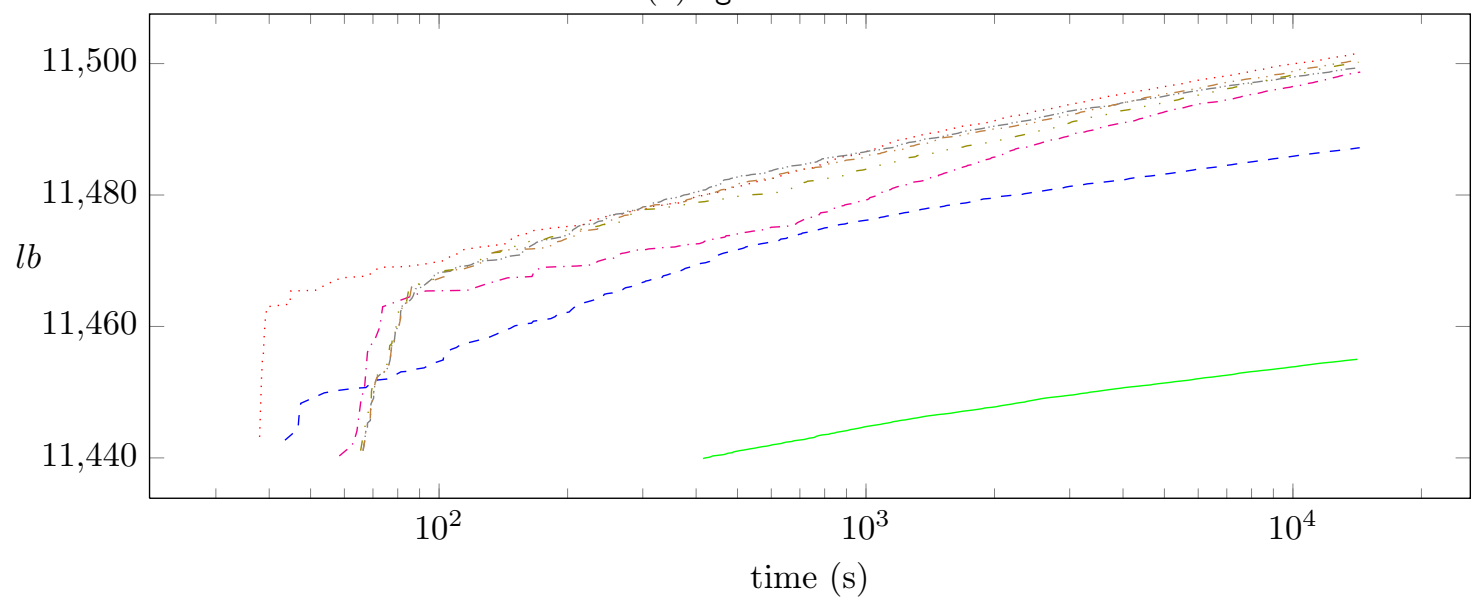

(c) egl-e4-c

Figure 4: Lower bounds over Time 


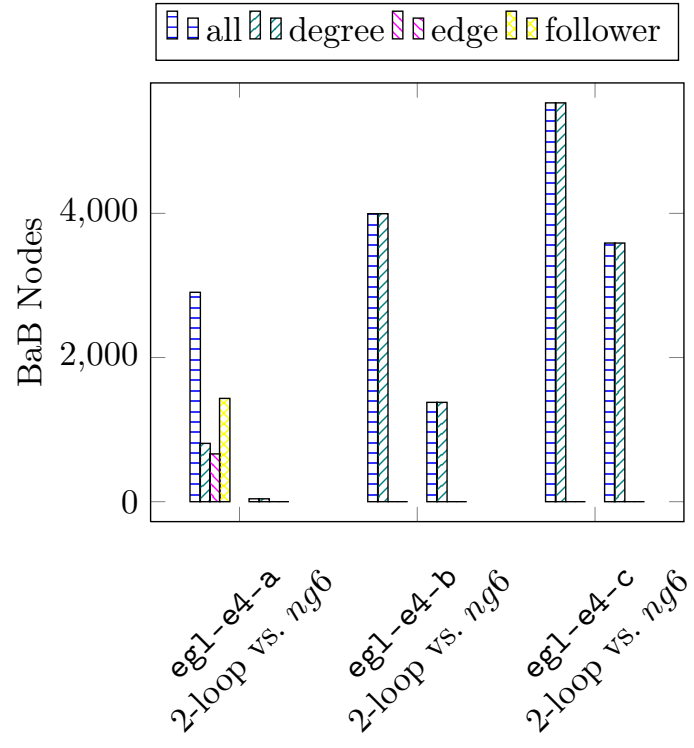

(a) Num. B\&B Nodes

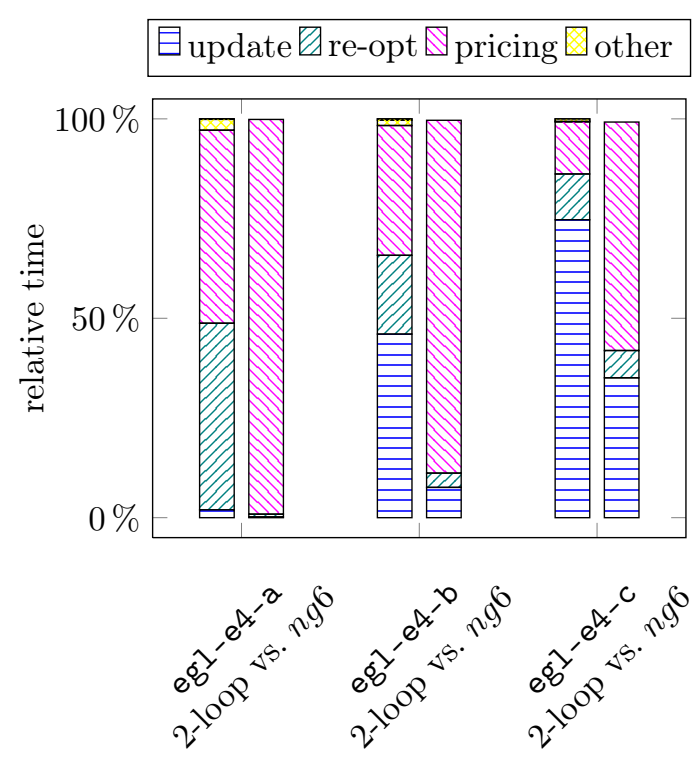

(b) Relative Times

Figure 5: Number of Branch-and-Bound Nodes/Decisions and Relative Times spent in Components

Because 2-loop is a relaxation of $n g 6$, it is always less time consuming. However, while many more nodes are solved with the 2-loop-free relaxation for the egl-lm-a group, the overall number of solved nodes becomes more and more comparable for the egl-lm-b and egl-lm-c groups. This explains well why the 2-loop-free relaxation is much more effective for instances with rather long routes and only very few routes (as for egl-lm-a).

For the instance egl-e4-a and the 2-loop relaxation, approximately half of the nodes result from branching on followers and non-followers, and the others from branching on node degrees and edge flows, respectively. Branching on follower information entails a network modification with computing, removing, and adding edges that represent shortest paths (see Bode and Irnich, 2012, Sect 4.3.2). The structural modification is done once and at the very beginning of each branch-and-bound node. Furthermore, least-cost deadheading paths must be computed in every pricing iteration. We expected that these modifications contribute with a significant computation time. However, during our experiments we found that when solving a branch-and-bound node, the most time-consuming steps are 'update' and 're-opt' the RMP, and 'pricing'. For none of the instances where branching on followers and non-followers was performed, the time for modifying the network reaches a relevant computation time. Hence, it is not considered explicitly, but subsumed in 'update' in the further analysis. For egl-e4-b and egl-e4-c, both algorithms branch almost exclusively on node degrees.

The relative percentage of time spent in these different components is shown in Figure $5 b$, For both algorithms, the time spent with pricing decreases when comparing the three groups egl-lm-a, egl-lm-b, and egl-lm-c. On the one hand, for the $n g 6$ relaxation, almost the entire time is spent on pricing for the instance egl-e4-a, while the relative time decreases to approximately $60 \%$ for the instance egl-e4-c. On the other hand, the 2-loop-free relaxation starts at about $60 \%$ pricing time for egl-e4-a; it decreases to almost $10 \%$ for the instance egl-e4-c.

At the same time, updating the RMP consumes relatively more time. At its extreme, updating takes about $80 \%$ of the time for the instance egl-e4-c when solved with the 2-loop-free relaxation. This time also increases for $n g 6$, but ends up at about only $30 \%$ for the instance egl-e4-c.

Effort for Updates. We further analyze the effort for updating the RMP and the pricing problem. The results are shown in Figure 6 . 


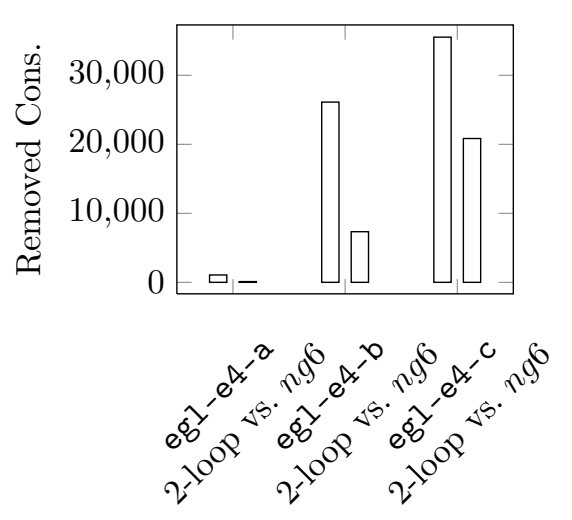

(a) Removed Constraints

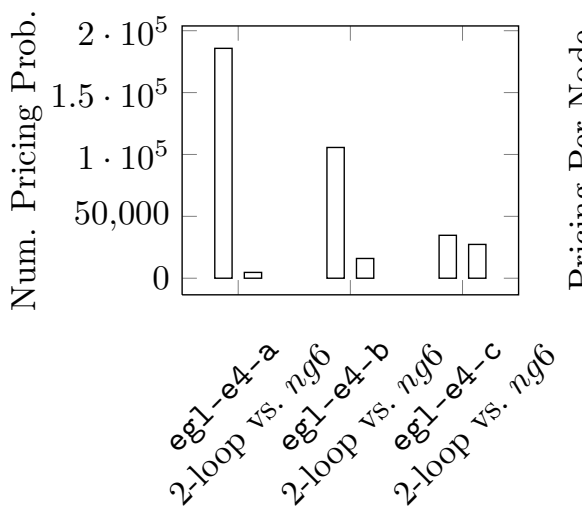

(b) Num. Pricing Problems

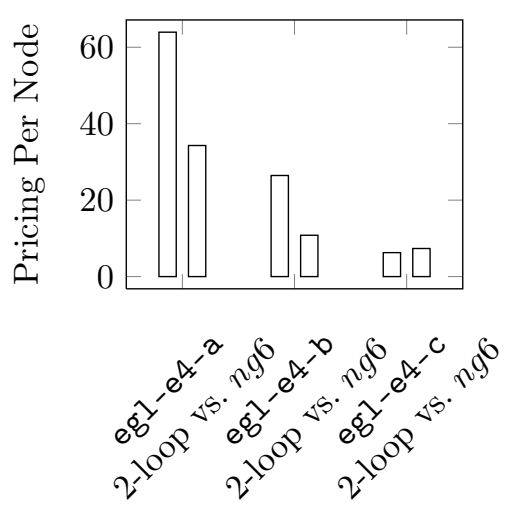

(c) Pricings Per Node

Figure 6: Number of Removed Constraints and Number of Pricing Problems overall/per Node

Updating the RMP consists of finding and adding the active branch-and-bound constraints regarding to node degrees and edge flows. The number of added and removed constraints for a RMP over the whole branch-and-price tree is approximately identical. Therefore, in Figure 6a, only the number of the 'removed' constraints of the RMP is plotted. Moreover, one must take care of having only compatible columns in the current RMP with regard to (non-)follower constraints and $n g$-route constraints. Incompatible columns are discarded by setting the lower and upper bound of this column to zero, without any complex modification of the RMP.

The Figures show that there is a strong correlation between the number of branch-and-bound nodes and the number of removed constraints, independent of the considered relaxation. The more branch-and-bound nodes are evaluated, the more constraints are removed and added. This additional effort explains partly the increasing time consumption within the RMP update.

On the other hand, while the relative time for updating the RMP increases, the relative time for pricing decreases. As shown in Figure 6b the number of solved pricing problems for the 2-loop relaxation decreases from about 250,000 for egl-e4-a to merely 50,000 for egl-e4-c. Related to the branch-and-bound nodes, the number of solved pricing problems per node also decreases from group a to c. Even so, the absolute number of solved pricing problems slightly increases for the $n g 6$-relaxation, the relative number of pricing problems per node decreases (see Figure 6c). In general, combining these relative numbers with the computation time needed to solve a single pricing problem, we end up with having many and computationally intensive pricings for egl-lm-a instances and less and computationally easier pricings for egl-lm-c instances. This explains the decreasing time consumption of the pricing part from groups a to c. Additionally, the $n g 6$ relaxation is a stronger relaxation because it includes 2-loop-free relaxation, which makes the pricing problems more difficult to solve. Therefore, the time consumption for pricing is always higher compared to 2-loop elimination.

Furthermore, the $n g 6$ relaxation as a stronger relaxation results in tighter lower bounds while having approximately the same number of pricing problems for the instance egl-e4-c. To conclude this section, the effect of fast pricing for the 2-loop relaxation is nullified if there is only a small number of columns to be priced out at each iteration. Then, the effect of a stronger relaxation is more important.

\subsection{Strong Branching and Integer Solution Results}

Strong branching is another technique often yielding better lower bounds when large branch-and-bound trees have to be explored. Instead of choosing a single variable/decision for branching, the idea is to evaluate several candidates for branching before taking the actual branching decision. Because evaluating candidates takes time, trees with less branch-and-bound nodes result. Nevertheless, the nodes provide relatively better lower bounds, which can be beneficial at the end. For a general discussion of strong branching techniques we refer to (Achterberg et al. 2005). 
We tested the $k$-loop relaxations for $k \in\{2,3,4\}$ and the $n g 6$ and $n g 7$ relaxations with five and ten candidates on the egl instances. We restrict strong branching to branch-and-bound nodes at levels not exceeding ten, i.e., with not more than ten nodes between the the root node and the node under consideration. Table 13 in the Appendix presents detailed results for computations with strong branching for all egl-lm-n instances, while Table 5 presents aggregated information.

Table 5: Aggregated Integer Results with Strong Branching for egl Instances

\begin{tabular}{|c|c|c|c|c|c|c|c|c|}
\hline & \multicolumn{2}{|c|}{ 2-loop } & \multicolumn{2}{|c|}{ 3-loop } & \multicolumn{2}{|c|}{ 4b2-loop } & \multicolumn{2}{|c|}{ 4b3-loop } \\
\hline & sb5 & sb10 & sb5 & sb10 & sb5 & sb10 & sb5 & sb10 \\
\hline Num. opt. sol. (all/a/b/c) & $5 / 4 / 1 / 0$ & $5 / 4 / 1 / 0$ & $5 / 4 / 1 / 0$ & $5 / 4 / 1 / 0$ & $5 / 4 / 1 / 0$ & $4 / 3 / 1 / 0$ & $4 / 3 / 1 / 0$ & $4 / 3 / 1 / 0$ \\
\hline Num. best $l b(\mathrm{all} / \mathrm{a} / \mathrm{b} / \mathrm{c})$ & $6 / 4 / 2 / 0$ & $9 / 8 / 1 / 0$ & $6 / 4 / 2 / 0$ & $5 / 4 / 1 / 0$ & $5 / 4 / 1 / 0$ & $6 / 3 / 1 / 2$ & $4 / 3 / 1 / 0$ & $4 / 3 / 1 / 0$ \\
\hline Average gap (\%) & 0,66 & 0,66 & 0,57 & 0,56 & 0,52 & 0,50 & 0,53 & 0,53 \\
\hline \multirow[t]{3}{*}{ Maximum gap (\%) } & 1,10 & 1,09 & 1,08 & 1,08 & 1,10 & 1,09 & 1,11 & 1,12 \\
\hline & \multicolumn{2}{|c|}{$n g 6$} & \multicolumn{2}{|c|}{$n g 7$} & & & & \\
\hline & sb5 & $\operatorname{sb} 10$ & $\operatorname{sb} 5$ & sb10 & & & & \\
\hline Num. opt. sol. (all/a/b/c) & $4 / 2 / 2 / 0$ & $4 / 2 / 2 / 0$ & $4 / 3 / 1 / 0$ & $4 / 2 / 2 / 0$ & & & & \\
\hline Num. best $l b($ all $/ \mathrm{a} / \mathrm{b} / \mathrm{c})$ & $10 / 2 / 6 / 2$ & $8 / 2 / 4 / 2$ & $8 / 3 / 1 / 4$ & $7 / 2 / 3 / 2$ & & & & \\
\hline Average gap (\%) & 0,43 & 0,44 & 0,45 & 0,45 & & & & \\
\hline Maximum gap (\%) & 1,07 & 1,09 & 1,08 & 1,08 & & & & \\
\hline
\end{tabular}

Comparing the number of optimal solutions, the $k$-loop and $n g$-relaxations are able to find about the same number of integer solutions. However, similar to the results in Section $5.4 k$-loop solves more instances of groups with higher capacity (i.e. egl-lm-a) to optimality. On the other hand, looking at the number of best lower bounds among all relaxation with strong branching, $n g 6$ and $n g 7$ with five or ten candidates perform always better, resulting also in smaller average and maximum gaps. Overall, several lower bounds are improved compared to the integer results without strong branching (egl-e3-b, egl-e4-c, egl-s3-a, and egl-s4-a).

\subsection{New Best Solutions for egl and bmcv Instances}

Compared to the best known results from the literature several lower bounds for both data set were improved. Tables 9, 12 and 13 summarize the results for the standard and large-scale egl instances, while Tables 10 and 11 present results for the bmcv instances. The dataset of the large-scale egl instances was proposed by (Brandão and Eglese, 2008) and contains instances with up to 255 nodes, 375 edges and 347 or 375 required edges. Values printed in bold indicate new best solutions. New best lower bounds were calculated for all large-scale egl instances and five standard egl instances (egl-e3-b, egl-e4-c, egl-s3-a, egl-s4-a, egl-s4-b). The instance egl-e2-b is solved to optimality for the first time. During preliminary experiments we found a new upper bound for the instance egl-e4-c. The corresponding solutions are shown in Section $\mathrm{D}$ of the Appendix.

For previously 33 unsolved bmcv instances, we obtained either better lower bounds or optimal solutions in 32 cases. In detail, better lower bounds were computed for six open C instances (C01, C09, C11, C12, $\mathrm{C} 15$ and $\mathrm{C} 23$ ) and four new optimal solutions were found (C04, C19, C21 and C24). However, compared to Bartolini et al. (2012), our lower bound for C18 is seven units worse. For the six remaining D instances, we computed three better lower bounds (D21, D23 and D24) and three optimal solutions (D08, D14 and D19). On the downside, we were not able to solve D07 which was solved to optimality by Bartolini et al. (2012). Furthermore, the root node for the instances D15 and D18 could not be solved within four hours with some relaxations. Five better lower bounds (E01, E09, E15, E18 and E23) and six optimal solutions (E11, E16, E10, E20, E21 and E24) were found for E instances. Note that for the instance E12 we ended up one unit worse than Bartolini et al. (2012). For F instances, we obtained three better lower bounds (F18, F19 and F23) and three optimal solutions (F04, F08 and F12). Furthermore, Bartolini et al. (2012) already mentioned that the objective value for bmcv instances is always a multiple of five because all edge costs are multiples of five. Therefore, they proved optimality for the the instance E21. Using the same argument, we can match the 
lower bounds of five additional instances with the upper bound (D23, E12, E18, E23 and F23). In the end, twelve standard egl instances and $14 \mathrm{bmcv}$ instances remain open.

\section{Conclusion}

In this work, different relaxations known from the node-routing context were adapted to solve the CARP with a branch-and-price approach. The adaptation to column generation-based approaches that price out new CARP tours over the original graph is by no means trivial, but is however attractive because it offers the application of highly effective pricing procedures that exploit the sparsity of the CARP network. Exploiting sparsity results in, compared to standard node-routing problems, a more intricate branching scheme, which in turn complicates the pricing. In essence, the effective approach of Bode and Irnich (2012) requires that the shortest-path pricing problem resulting from a relaxation must be able to handle two sets of tasks: One set $\mathcal{T}^{E}$ models elementary routes and the other set $\mathcal{T}^{B}$ incorporates non-follower constraints implied by the branching scheme. While for $\mathcal{T}^{E}$ any relaxation of elementary routes is applicable, routes must be exactly 2-loop-free regarding to tasks in $\mathcal{T}^{B}$.

First, we have adapted the $n g$-route relaxations (Baldacci et al., 2011b) and the $k$-loop-free relaxations (Irnich and Villeneuve, 2006) leading to combined $n g$-route 2-loop-free relaxations and combined $(k, 2)$ loop-free relaxations. For the latter, a new labeling algorithm was developed. Its key component are strong dominance rules that we derived, based on new worst-case complexity results guaranteeing that, for a fixed parameter $k$, the number of labels to consider never exceeds $(k-1) !(k+1)$ ! times the size of the underlying state space. Concluding, a pricing problem resulting from the $(k, 2)$-loop-free relaxation with $k$ fixed can be solved in $\mathcal{O}(C \cdot(|E|+|V| \log |V|))$ time, where $C$ is the vehicle capacity and $\mathcal{O}(|E|+|V| \log |V|)$ the best known bound for solving shortest-path problems using Dijkstra's algorithm.

Second, we integrated acceleration techniques for the heuristic and exact solution of the pricing problems. In particular, bi-directional labeling (Righini and Salani, 2006) and bounding (Baldacci et al. 2009) techniques were modified to fit with all relaxations.

Third, we presented a comprehensive computational study where the performance of the acceleration techniques, the quality of the bounds (lower bounds at the root node and over time in branch-and-price), and the overall performance of different branch-and-price algorithms were analyzed. Moreover, we tried to characterize which type of relaxation and acceleration technique is best suited to solve a specific group of instances. The standard instances egl of Eglese and Li (1992) and bmcv of Beullens et al. (2003) were used for that purpose. In summary, reasonable parameters are $k \in\{2,3,4\}$ for $(k, 2)$-loop elimination and $n_{n g} \in\{5,6,7\}$ for the maximum size of neighborhoods in $n g$-route relaxations. Bounding with the 2-loop-free relaxation is generally sufficient, stronger relaxations do not pay off. For the entire branch-andprice, bi-directional labeling alone accelerates better than bounding alone, but the combination of both is often even more effective providing acceleration factors of approximately four for $n g$-route relaxations and $(3,2)$-loop elimination, and factor eight for $(4,2)$-loop elimination. The study of lower bounds provided by the linear relaxations with $(k, 2)$-loop elimination and $n g$-routes shows that neither relaxation outperforms the others on all instances. Concerning groups of instances, $k$-loop-free relaxations often work better for instances utilizing fewer vehicles, higher capacities, and relatively long routes. The opposite is true for $n g$-route relaxations working best when solutions comprise more vehicles with relatively shorter routes.

Overall, the newly considered relaxations with loop elimination for $k=3$ and $k=4$ as well as the use of the $n g$-route relaxations outperformed the already remarkable results with elementary routes presented by Bartolini et al. (2012) and with the pure 2-loop-free relaxation presented by Bode and Irnich (2012). The different branch-and-price algorithms delivered 22 new best lower bounds of the egl and bmcv benchmark sets, and improved all lower bounds for the twelve large-scale egl instances by Martinelli et al. (2011b). Finally, 20 previously open instances, one for the standard egl and 19 for bmcv benchmark set, are solved to optimality for the first time. 


\section{References}

Achterberg, T., Koch, T., and Martin, A. (2005). Branching rules revisited. Operations Research Letters, 33(1), 42-54.

Ahr, D. (2004). Contributions to Multiple Postmen Problems. Phd dissertation, Department of Computer Science, Heidelberg University, Heidelberg, Germany.

Ahuja, R., Magnanti, T., and Orlin, J. (1993). Network Flows: Theory, Algorithms, and Applications. Prentice Hall, Englewood Cliffs, New Jersey.

Baldacci, R. and Maniezzo, V. (2006). Exact methods based on node-routing formulations for undirected arc-routing problems. Networks, 47(1), 52-60.

Baldacci, R., Mingozzi, A., and Roberti, R. (2009). Solving the vehicle routing problem with time windows using new state space relaxation and pricing strategies. Presented at AIRO 2008, EURO 2009, ISMP 2009, and AIRO 2009.

Baldacci, R., Mingozzi, A., and Roberti, R. (2011a). Dynamic ng-path relaxation. Presentation at the ROUTE 2011 conference. http://www.uv.es/route2011/Roberti.pdf.

Baldacci, R., Mingozzi, A., and Roberti, R. (2011b). New Route Relaxation and Pricing Strategies for the Vehicle Routing Problem. Operations Research, 59(5), 1269-1283.

Baldacci, R., Mingozzi, A., and Roberti, R. (2011c). New state-space relaxations for solving the traveling salesman problem with time windows. INFORMS Journal on Computing.

Bartolini, E., Cordeau, J.-F., and Laporte, G. (2012). Improved lower bounds and exact algorithm for the capacitated arc routing problem. Mathematical Programming, pages 1-44.

Belenguer, J. and Benavent, E. (1998). The capacitated arc routing problem: Valid inequalities and facets. Computational Optimization and Applications, 10(2), 165-187.

Belenguer, J. and Benavent, E. (2003). A cutting plane algorithm for the capacitated arc routing problem. Computers \& Operations Research, 30, 705-728.

Belenguer, J.-M., Benevent, E., and Irnich, S. (2013). The capacitated arc routing problem: Exact algorithms. In Corberán and Laporte (2013), chapter 9. (In preparation.).

Ben Amor, H., Desrosiers, J., and Valério de Carvalho, J. (2006). Dual-optimal inequalities for stabilized column generation. Operations Research, 54(3), 454-463.

Benavent, E., Campos, V., Corberán, A., and Mota, E. (1992). The capacitated arc routing problem: Lower bounds. Networks, 22, 669-690.

Beullens, P., Muyldermans, L., Cattrysse, D., and Van Oudheusden, D. (2003). A guided local search heuristic for the capacitated arc routing problem. European Journal of Operational Research, 147(3), 629-643.

Bode, C. and Irnich, S. (2012). Cut-first branch-and-price-second for the capacitated arc-routing problem. Operations Research, (accepted for publication). Available at http://logistik.bwl.uni-mainz.de/158.php.

Brandão, J. and Eglese, R. (2008). A deterministic tabu search algorithm for the capacitated arc routing problem. Computers ES Operations Research, 35(4), 1112-1126.

Corberán, A. and Laporte, G., editors (2013). Arc Routing: Problems, Methods and Applications. MOS-SIAM Series on Optimization. SIAM, Philadelphia. (In preparation.).

Corberán, A. and Prins, C. (2010). Recent results on arc routing problems: An annotated bibliography. Networks, 56(1).

Desaulniers, G., Desrosiers, J., and Solomon, M. (2002). Accelerating strategies in column generation for vehicle routing and crew scheduling problems. In C. Ribeiro and P. Hansen, editors, Essays and Surveys in Metaheuristics, Operations Research/Computer Science Interfaces Series, chapter 14, pages 309-324. Kluwer, Boston.

Desaulniers, G., Desrosiers, J., and Solomon, M., editors (2005). Column Generation. Springer, New York, NY.

Desaulniers, G., Lessard, F., and Hadjar, A. (2008). Tabu search, partial elementarity, and generalized k-path inequalities for the vehicle routing problem with time windows. Transportation Science, 42(3), 387-404.

Dror, M., editor (2000). Arc Routing: Theory, Solutions and Applications. Kluwer, Boston.

Eglese, R. and Li, L. (1992). Efficient routeing for winter gritting. Journal of the Operational Research Society, 43(11), $1031-1034$.

Fu, H., Mei, Y., Tang, K., and Zhu, Y. (2010). Memetic algorithm with heuristic candidate list strategy for capacitated arc routing problem. In Evolutionary Computation (CEC), 2010 IEEE Congress on, pages 1-8. IEEE.

Fukasawa, R., Longo, H., Lysgaard, J., Poggi de Aragão, M., Reis, M., Uchoa, E., and Werneck, R. F. (2006). Robust branchand-cut-and-price for the capacitated vehicle routing problem. Mathematical Programming, Series A, 106(3), 491-511.

Gómez-Cabrero, D., Belenguer, J., and Benavent, E. (2005). Cutting planes and column generation for the capacitated arc routing problem. presented at ORP3, Valencia, Spain.

Golden, B. and Wong, R. (1981). Capacitated arc routing problems. Networks, 11, 305-315.

Irnich, S. and Desaulniers, G. (2005). Shortest path problems with resource constraints. In Desaulniers et al. (2005), chapter 2, pages $33-65$

Irnich, S. and Villeneuve, D. (2006). The shortest path problem with resource constraints and $k$-cycle elimination for $k \geq 3$. INFORMS Journal on Computing, 18(3), 391-406.

Kohl, N. (1995). Exact methods for Time Constrained Routing and Related Scheduling Problems. Dissertation, Department of Mathematical Modelling, Technical University of Denmark.

Lacomme, P., Prins, C., and Ramdane-Chérif, W. (2001). A genetic algorithm for the capacitated arc routing problem and its extensions. In E. J. Boers, S. Cagnoni, J. Gottlieb, E. Hart, P. L. Lanzi, G. Raidl, R. E. Smith, and H. Tijink, editors, Applications of Evolutionary Computing. EvoWorkshops2001: EvoCOP, EvoFlight, EvoIASP, EvoLearn, and EvoSTIM. Proceedings, volume 2037 of LNCS, pages 473-483, Como, Italy. Springer-Verlag. 
Larsen, J. (1999). Parallelization of the Vehicle Routing Problem with Time Windows. Ph.D. thesis, Department of Mathematical Modelling, Technical University of Denmark.

Lübbecke, M. and Desrosiers, J. (2005). Selected topics in column generation. Operations Research, 53(6), 1007-1023.

Letchford, A. (1997). Polyhedral results for some arc routing problems. Phd dissertation, Department of Management Science, Lancaster University.

Letchford, A. N. and Oukil, A. (2009). Exploiting sparsity in pricing routines for the capacitated arc routing problem. Computers \& Operations Research, 36(7), 2320-2327.

Longo, H., de Aragao, M., and Uchoa, E. (2006). Solving capacitated arc routing probelms using a transformation to the CVRP. Computers \& Operations Research, 33(6), 1823-1837.

Martinelli, R., Pecin, D., Poggi, M., and Longo, H. (2011a). A branch-cut-and-price algorithm for the capacitated arc routing problem. In P. Pardalos and S. Rebennack, editors, Experimental Algorithms, volume 6630 of Lecture Notes in Computer Science, pages 315-326. Springer Berlin / Heidelberg.

Martinelli, R., Poggi de Argão, M., and Subramanian, A. (2011b). Improved bounds for large scale capacitated arc routing problem. Preprint submitted to computers \& operations research, Departamento de Informática, Rio de Janeiro, RJ 22453900, Brazil.

McGill, R., Tukey, J. W., and Larsen, W. A. (1978). Variations of box plots. The American Statistician, 32(1), 12-16.

Mei, Y., Tang, K., and Yao, X. (2009). A global repair operator for capacitated arc routing problem. Systems, Man, and Cybernetics, Part B: Cybernetics, IEEE Transactions on, 39(3), 723-734.

Mingozzi, A., Bianco, L., and Ricciardelli, S. (1997). Dynamic programming strategies for the traveling salesman problem with time window and precedence constraints. Operations Research, 45(3), 365-377.

Muyldermans, L. (2012). Personal Communication.

Polacek, M., Doerner, K., Hartl, R., and Maniezzo, V. (2008). A variable neighborhood search for the capacitated arc routing problem with intermediate facilities. Journal of Heuristics, 14, 405-423. 10.1007/s10732-007-9050-2.

Prins, C. (2013). The capacitated arc routing problem: Heuristics. In Corberán and Laporte (2013), chapter 7. (In preparation.).

Righini, G. and Salani, M. (2006). Bounded bidirectional dynamic programming for the elementary shortest path problem with resource constraints. Discrete Optimization, 3(3), 255-273.

Santos, L., Coutinho-Rodrigues, J., and Current, J. R. (2010). An improved ant colony optimization based algorithm for the capacitated arc routing problem. Transportation Research Part B, 44(2), $246-266$.

Tang, K., Mei, Y., and Yao, X. (2009). Memetic algorithm with extended neighborhood search for capacitated arc routing problems. Evolutionary Computation, IEEE Transactions on, 13(5), 1151-1166.

Vazirani, V. (2001). Approximation Algorithms. Springer, New York.

Wøhlk, S. (2008). A decade of capacitated arc routing. In R. Sharda, S. Voß, B. Golden, S. Raghavan, and E. Wasil, editors, The Vehicle Routing Problem: Latest Advances and New Challenges, volume 43 of Operations Research/Computer Science Interfaces Series, pages 29-48. Springer. 10.1007/978-0-387-77778-8 2. 


\section{Appendix}

\section{A. Proofs}

This section contains proofs of the worst-case complexity results for combined $\left(k_{1}, k_{2}\right)$-loop elimination as introduced in Section 3.4 of the paper. Note that the proofs follow similar ideas as discussed in the first article on $k$-cycle elimination (focused on node-routing applications) and we refer the reader to this (Irnich and Villeneuve, 2006) for a more detailed motivation.

Theorem. Let the first set of tasks required to be $k_{1}$-loop-free and the second set of tasks to be $k_{2}$-loop-free. Then the maximum number of different set forms needed to represent any intersection $H\left(P_{1}\right) \cap H\left(P_{2}\right) \cap \cdots \cap$ $H\left(P_{l}\right)$ of self-hole sets of any set of $l$ paths is $\left(k_{1}-1\right) !^{2} \cdot\left(k_{2}-1\right) !^{2} \cdot\left(\begin{array}{c}\left(k_{1}-1\right)+\left(k_{2}-1\right) \\ k_{1}-1\end{array}\right)$. This bound is tight.

Proof. Define $I_{1}(s), I_{2}(s)$ of an arbitrary set forms $s=\left(s_{1}^{1}, \ldots, s_{k_{1}-1}^{1}\right)\left(s_{1}^{2}, \ldots, s_{k_{2}-1}^{2}\right)$ with $s_{i}^{1} \in \mathcal{T}^{1} \cup\{\cdot\}$ and $s_{j}^{2} \in \mathcal{T}^{2} \cup\{\cdot\}$ as

$$
I_{1}(s):=\left\{i \in\left\{1, \ldots, k_{1}-1\right\} \mid s_{i}^{1}=\cdot\right\} \quad \text { and } \quad I_{2}(s):=\left\{j \in\left\{1, \ldots, k_{2}-1\right\} \mid s_{j}^{2}=\cdot\right\}
$$

Let the $I(s)=\left(I_{1}(s), I_{2}(s)\right)$ be the type of an arbitrary set forms $s$. To shorten the notation we will write $I=\left(I_{1}, I_{2}\right)$ instead of $I(s)=\left(I_{1}(s), I_{2}(s)\right)$. We denote by $n_{k_{1}, k_{2}}(I)$ the maximum number of different set forms that can be generated from a set form of type $I$ by intersection with arbitrarily chosen self-hole sets. $n_{k_{1}, k_{2}}$ is defined on all subsets $I=\left(I_{1}, I_{2}\right) \subseteq\left(\left\{1, \ldots, k_{1}-1\right\},\left\{1, \ldots, k_{2}-1\right\}\right)$. The following recurrences are valid for $n_{k_{1}, k_{2}}$ :

$$
\begin{aligned}
& n_{k_{1}, k_{2}}(\emptyset, \emptyset)=1 \\
& n_{k_{1}, k_{2}}(I)=\sum_{i \in I_{1}}\left(k_{1}-i\right) n_{k_{1}, k_{2}}\left(I_{1} \backslash\{i\}, I_{2}\right)+\sum_{j \in I_{2}}\left(k_{2}-j\right) n_{k_{1}, k_{2}}\left(I_{1}, I_{2} \backslash\{j\}\right) \\
& \forall I_{1} \subseteq\left\{1, \ldots, k_{1}-1\right\} \text { and } I_{2} \subseteq\left\{1, \ldots, k_{2}-1\right\} \text { and } I \neq(\emptyset, \emptyset)
\end{aligned}
$$

The first equation is clear. The second equation is implied by the intersection operation. For each position $l$ there are either $k_{1}-l$ or $k_{2}-l$ different possibilities to place an element of the self-hole set at this position. This recurrence is solved by

$$
n_{k_{1}, k_{2}}(I)=\left[\left|I_{1}\right| ! \prod_{i \in I_{1}}\left(k_{1}-i\right)\right]\left[\left|I_{2}\right| ! \prod_{j \in I_{2}}\left(k_{2}-j\right)\right]\left[\left(\begin{array}{c}
\left|I_{1}\right|+\left|I_{2}\right| \\
\left|I_{1}\right|
\end{array}\right)\right] .
$$

This can be seen by induction on the cardinality of $I$. For $I=(\emptyset, \emptyset)$ this gives $n_{k_{1}, k_{2}}(\emptyset, \emptyset)=1$, which is 
correct. Now assume, that the above equality is true for all subsets with cardinality $|I|-1$.

$$
\begin{aligned}
n_{k_{1}, k_{2}}(I)= & \sum_{i \in I_{1}}\left(k_{1}-i\right) n_{k_{1}, k_{2}}\left(I_{1} \backslash\{i\}, I_{2}\right)+\sum_{j \in I_{2}}\left(k_{2}-j\right) n_{k_{1}, k_{2}}\left(I_{1}, I_{2} \backslash\{j\}\right) \\
= & \sum_{i \in I_{1}}\left(k_{1}-i\right)\left(\left|I_{1}\right|-1\right) ! \prod_{l \in I_{1} \backslash\{i\}}\left(k_{1}-l\right)\left|I_{2}\right| ! \prod_{m \in I_{2}}\left(k_{2}-m\right)\left(\begin{array}{c}
\left|I_{1}\right|+\left|I_{2}\right|-1 \\
\left|I_{1}\right|-1
\end{array}\right)+ \\
& \sum_{j \in I_{2}}\left(k_{2}-j\right)\left|I_{1}\right| ! \prod_{l \in I_{1}}\left(k_{1}-l\right)\left(\left|I_{2}\right|-1\right) ! \prod_{m \in I_{2} \backslash\{j\}}\left(k_{2}-m\right)\left(\begin{array}{c}
\left|I_{1}\right|+\left|I_{2}\right|-1 \\
\left|I_{1}\right|
\end{array}\right) \\
= & \sum_{i \in I_{1}}\left(\left|I_{1}\right|-1\right) !\left(k_{1}-i\right) \prod_{l \in I_{1} \backslash\{i\}}\left(k_{1}-l\right)\left|I_{2}\right| ! \prod_{m \in I_{2}}\left(k_{2}-m\right)\left(\begin{array}{c}
\left|I_{1}\right|+\left|I_{2}\right|-1 \\
\left|I_{1}\right|-1
\end{array}\right)+ \\
& \sum_{j \in I_{2}}\left|I_{1}\right| ! \prod_{l \in I_{1}}\left(k_{1}-l\right)\left(\left|I_{2}\right|-1\right) !\left(k_{2}-j\right) \prod_{m \in I_{2} \backslash\{j\}}\left(k_{2}-m\right)\left(\begin{array}{c}
\left|I_{1}\right|+\left|I_{2}\right|-1 \\
\left|I_{1}\right|
\end{array}\right) \\
= & \sum_{i \in I_{1}}\left(\left|I_{1}\right|-1\right) ! \prod_{l \in I_{1}}\left(k_{1}-l\right)\left|I_{2}\right| ! \prod_{m \in I_{2}}\left(k_{2}-m\right)\left(\begin{array}{c}
\left|I_{1}\right|+\left|I_{2}\right|-1 \\
\left|I_{1}\right|-1
\end{array}\right)+ \\
& \sum_{j \in I_{2}}\left|I_{1}\right| ! \prod_{l \in I_{1}}\left(k_{1}-l\right)\left(\left|I_{2}\right|-1\right) ! \prod_{m \in I_{2}}\left(k_{2}-m\right)\left(\begin{array}{c}
\left|I_{1}\right|+\left|I_{2}\right|-1 \\
\left|I_{1}\right|
\end{array}\right) \\
= & \prod_{l \in I_{1}}\left(k_{1}-l\right) \prod_{m \in I_{2}}\left(k_{2}-m\right)\left[\sum_{i \in I_{1}}\left(\left|I_{1}\right|-1\right) !\left|I_{2}\right| !\left(\begin{array}{c}
\left|I_{1}\right|+\left|I_{2}\right|-1 \\
\left|I_{1}\right|-1
\end{array}\right)+\sum_{j \in I_{2}}\left|I_{1}\right| !\left(\left|I_{2}\right|-1\right) !\left(\begin{array}{c}
\left|I_{1}\right|+\left|I_{2}\right|-1 \\
\left|I_{1}\right|
\end{array}\right)\right] \\
= & \left|I_{1}\right| ! \prod_{l \in I_{1}}\left(k_{1}-l\right)\left|I_{2}\right| ! \prod_{m \in I_{2}}\left(k_{2}-m\right)\left[\left(\begin{array}{c}
\left|I_{1}\right|+\left|I_{2}\right|-1 \\
\left|I_{1}\right|-1
\end{array}\right)+\left(\begin{array}{c}
\left|I_{1}\right|+\left|I_{2}\right|-1 \\
\left|I_{1}\right|
\end{array}\right)\right] \\
= & \left|I_{1}\right| ! \prod_{l \in I_{1}}\left(k_{1}-l\right)\left|I_{2}\right| ! \prod_{m \in I_{2}}\left(k_{2}-m\right)\left(\begin{array}{c}
\left|I_{1}\right|+\left|I_{2}\right| \\
\left|I_{1}\right|
\end{array}\right)
\end{aligned}
$$

The above expression proves that we can get at most $\left(k_{1}-1\right) !^{2} \cdot\left(k_{2}-1\right) !^{2} \cdot\left(\begin{array}{c}\left(k_{1}-1\right)+\left(k_{2}-1\right) \\ k_{1}-1\end{array}\right)$ different elements in the intersection. To show that this bound is tight we choose any $\bar{k}=k_{1}+k_{2}$ different paths $P_{1}, \ldots, P_{\bar{k}}$ with disjoint predecessor tasks on both task-sets. Then the intersection of the corresponding self-hole sets consists of exactly $\left(k_{1}-1\right) !^{2} \cdot\left(k_{2}-1\right) !^{2} \cdot\left(\begin{array}{c}\left(k_{1}-1\right)+\left(k_{2}-1\right) \\ k_{1}-1\end{array}\right)$ elements.

Theorem. A collection of $s$ dominating paths $P_{1} \prec_{\text {dom }} P_{2} \prec_{\text {dom }} \ldots \prec_{\text {dom }} P_{s}$ with identical resource vectors ending at the same node is given. Let the intersections of the corresponding self-hole sets $H\left(P_{1}\right), H\left(P_{2}\right), \ldots, H\left(P_{s}\right)$ form a properly decreasing chain, i.e. $H\left(P_{1}\right) \supsetneq H\left(P_{1}\right) \cap H\left(P_{2}\right) \supsetneq \cdots \supsetneq \cap_{i=1}^{s} H\left(P_{i}\right)$. Then, the length $q$ of the properly decreasing chain is bounded by $\alpha\left(k_{1}, k_{2}\right)=\left(k_{1}+k_{2}-1\right) \cdot\left(k_{1}-1\right) !^{2} \cdot\left(k_{2}-1\right) !^{2} \cdot\left(\begin{array}{c}\left(k_{1}-1\right)+\left(k_{2}-1\right) \\ k_{1}-1\end{array}\right)$.

Proof. Every new element of the chain is a result of the intersections made before with one new intersection with a self-hole set $H\left(P_{i}\right)$. From Theorem 1 we know that there are at maximum $\left(k_{1}-1\right) !^{2} \cdot\left(k_{2}-1\right) !^{2}$. $\left(\begin{array}{c}\left(k_{1}-1\right)+\left(k_{2}-1\right) \\ k_{1}-1\end{array}\right)$ different set forms in such an intersection. Every set form has $\left(k_{1}-1\right)+\left(k_{2}-1\right)$ entries which results in $\left[\left(k_{1}-1\right)+\left(k_{2}-1\right)\right]\left(k_{1}-1\right) !^{2} \cdot\left(k_{2}-1\right) !^{2} \cdot\left({ }^{\left(k_{1}-1\right)+\left(k_{2}-1\right)}\right)$ different entries in total. The computation of the intersection there are two possible operations:

1. A new set form is generated, where a previously free entry $\cdot$ is specified by an element $t^{1} \in \mathcal{T}^{1}$ or $t^{2} \in \mathcal{T}^{2}$. There exists at most $\left[\left(k_{1}-1\right)+\left(k_{2}-1\right)\right] \cdot\left(k_{1}-1\right) !^{2} \cdot\left(k_{2}-1\right) !^{2} \cdot\left(\begin{array}{c}\left(k_{1}-1\right)+\left(k_{2}-1\right) \\ k_{1}-1\end{array}\right)$ possible entries to specify.

2. On the other hand, a set form can be deleted. This can happen at most $\left(k_{1}-1\right) !^{2} \cdot\left(k_{2}-1\right) !^{2}$. $\left(\begin{array}{c}\left(k_{1}-1\right)+\left(k_{2}-1\right) \\ k_{1}-1\end{array}\right)$ times.

Since each intersection performs at least one of the above operations, this yields to an upper bound of $\left[\left(k_{1}-1\right)+\left(k_{2}-1\right)+1\right]\left(k_{1}-1\right) !^{2} \cdot\left(k_{2}-1\right) !^{2} \cdot\left(\begin{array}{c}\left(k_{1}-1\right)+\left(k_{2}-1\right) \\ k_{1}-1\end{array}\right)$. 


\section{B. Tables}

Linear Relaxation Results. The Tables $6 / 8$ present the linear relaxation results for the egl and bmcv instances. The meaning of the table entries are as follows:

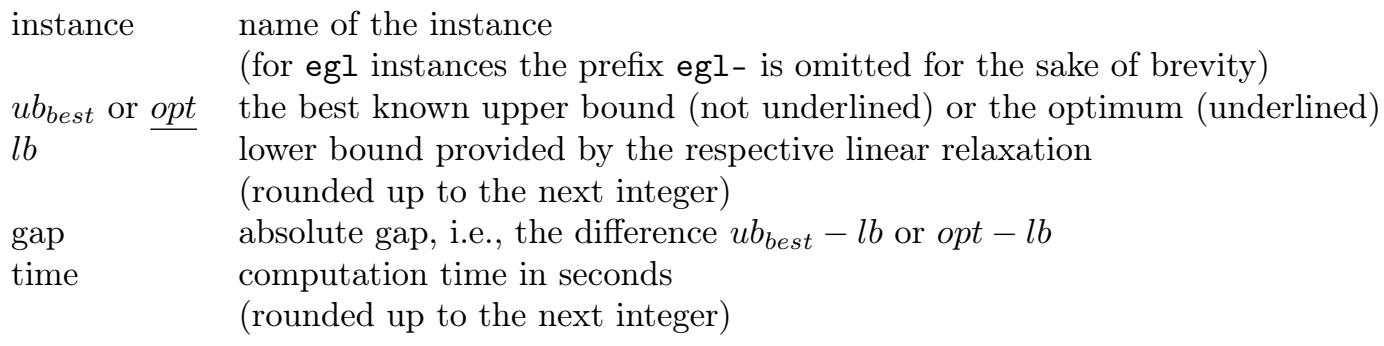

Integer Solution Results. The Tables $9 \sqrt{11}$ present the integer results for the egl and bmcv instances. The meaning of the table entries are as follows:

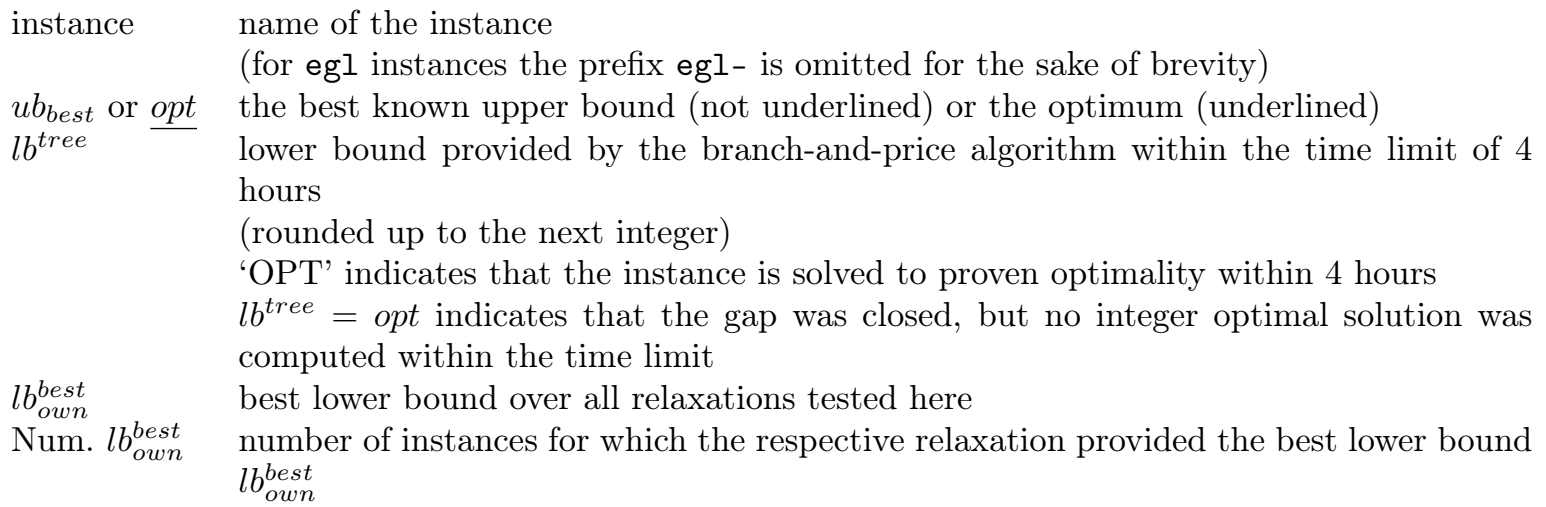

Lower bounds written in bold indicate that that this bound is a new best bound exceeding the best known lower bounds from the literature. The upper bounds $u b=11529$ for the instance egl-e4-c and $u b=4650$ for the bmcv instance E11 (written in bold also) result from new best integer solutions found with branchand-price.

The Table 12 presents the integer results for the large-scale egl instances. The meaning of the table entries are as follows:

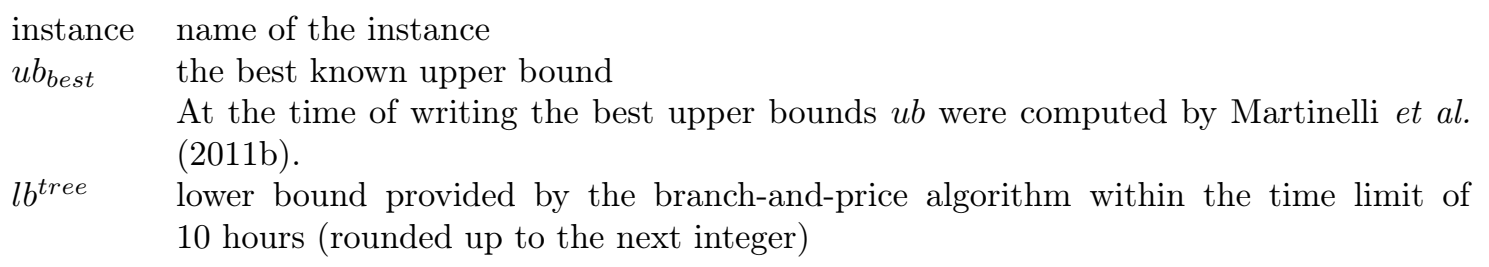

Lower bounds written in bold indicate that that this bound is a new best bound exceeding the best known lower bounds from the literature. 


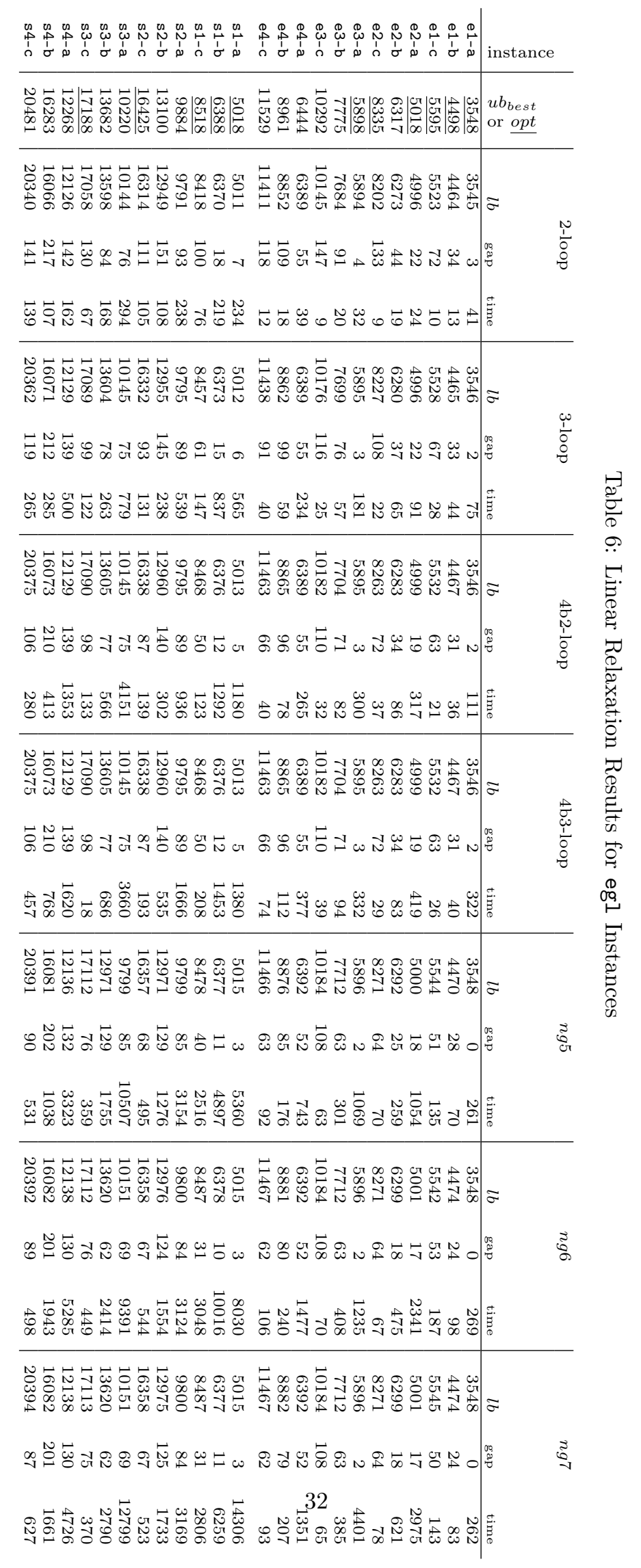




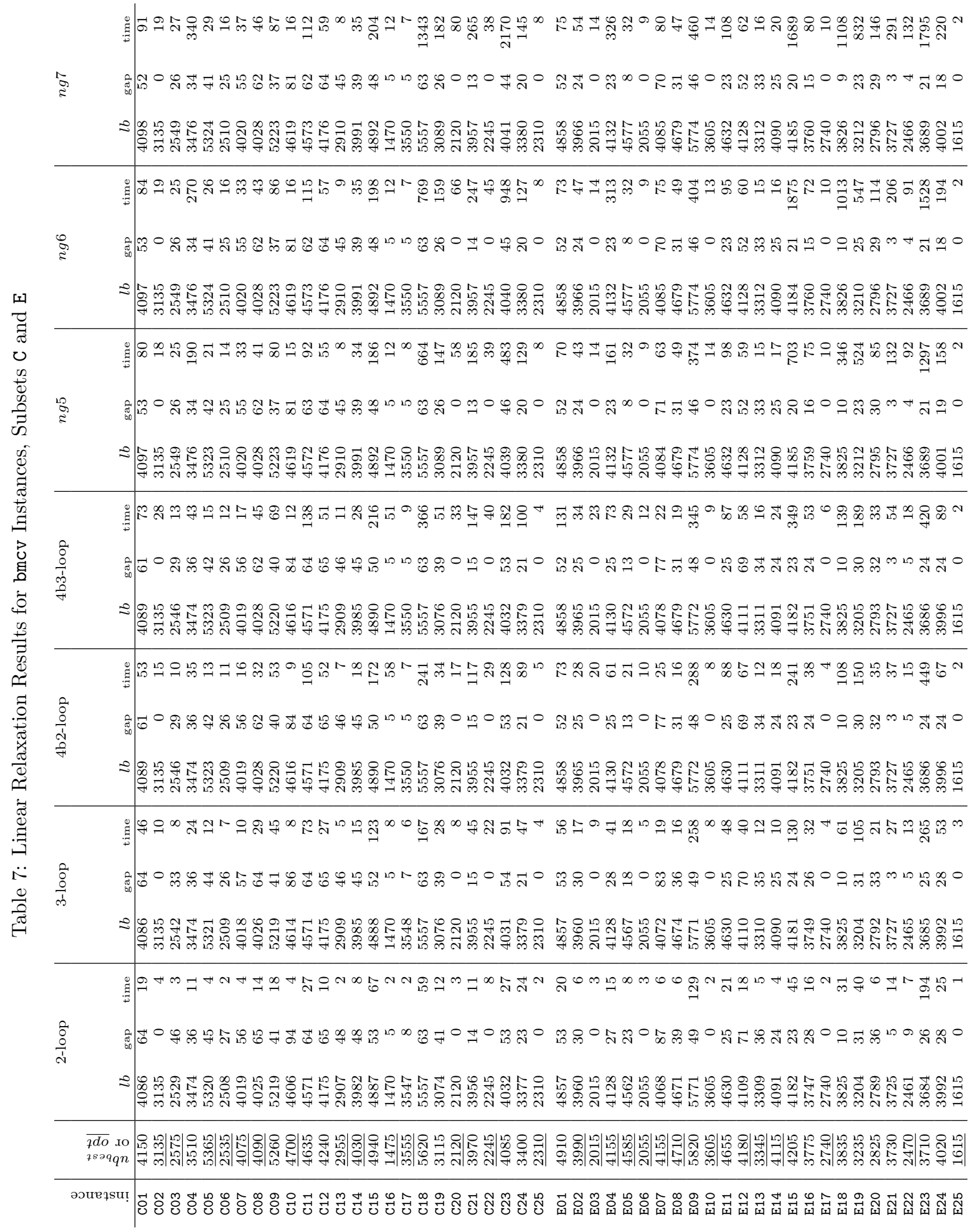




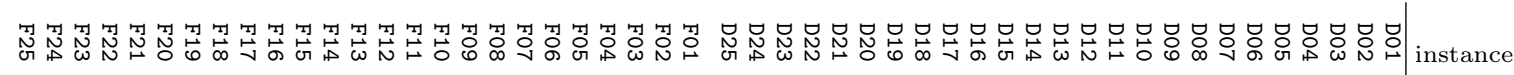

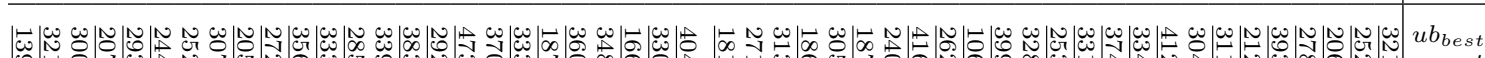

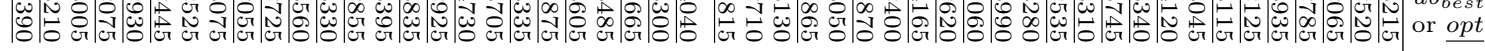

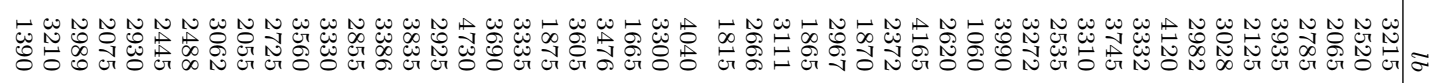

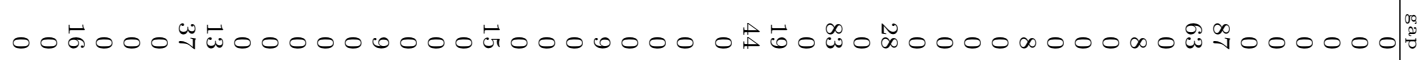

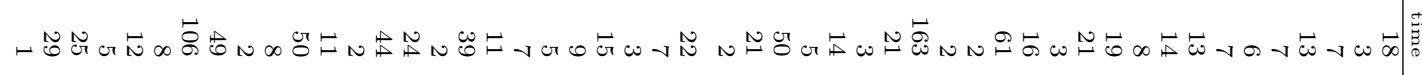

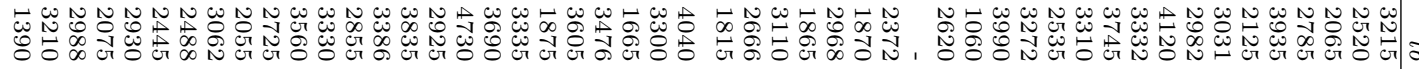
0 O V 000 U

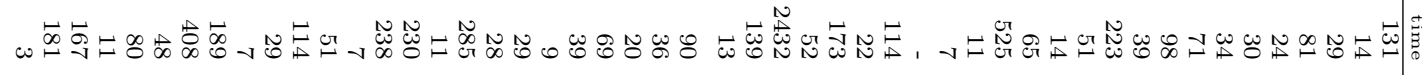

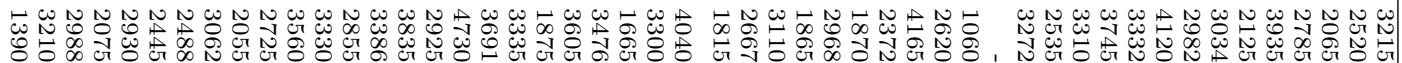

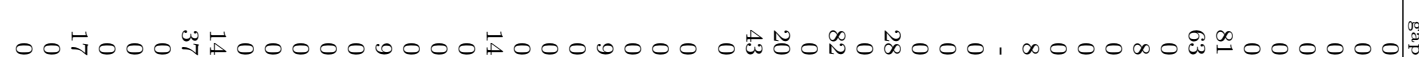

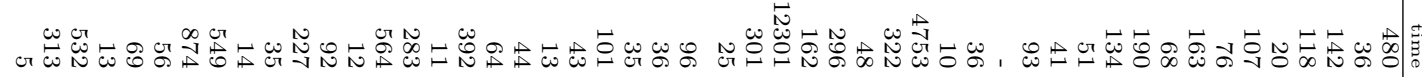

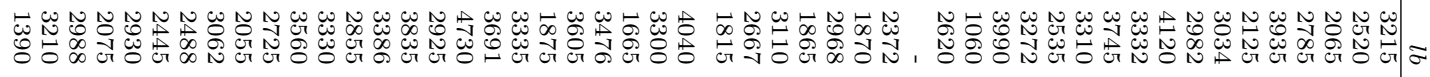

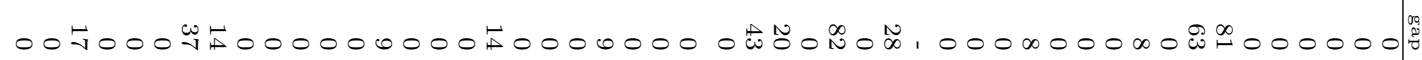

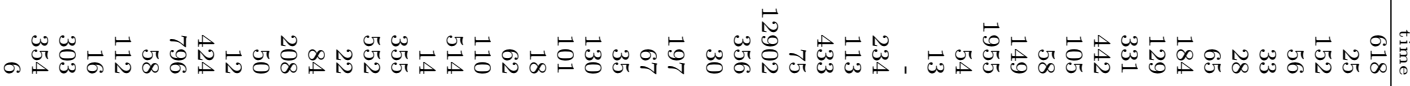

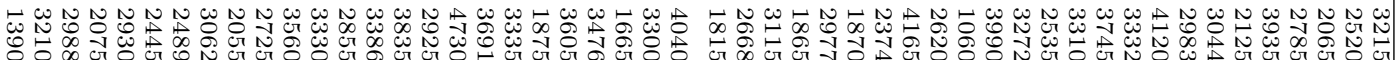

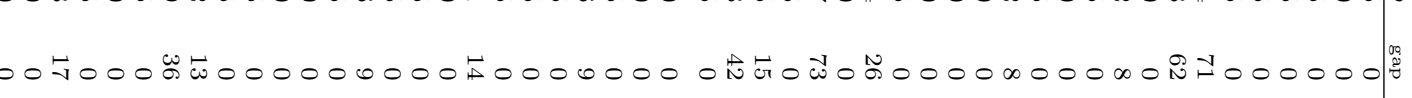

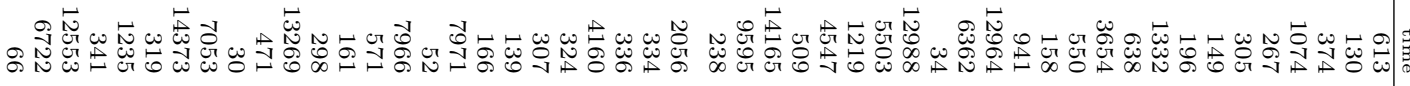

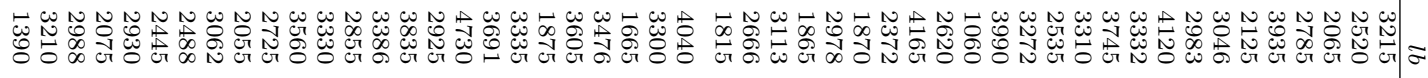

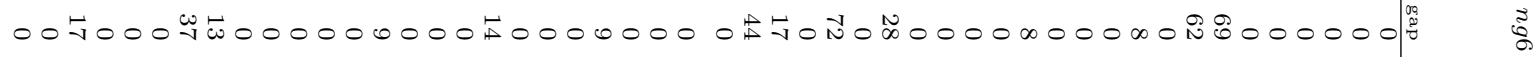

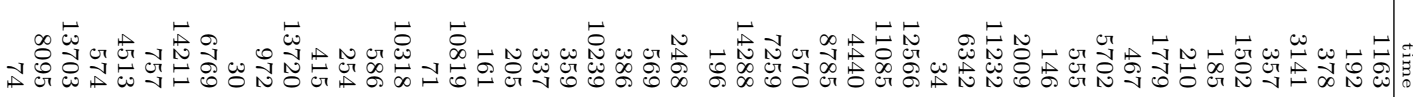

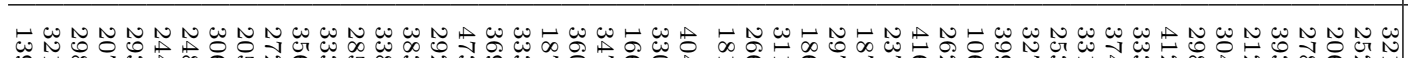

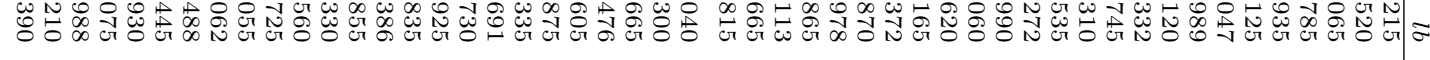

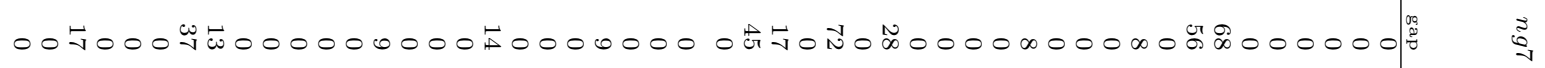

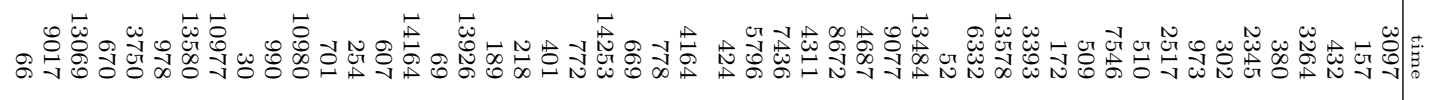


Table 9: Integer Results for egl Instances

\begin{tabular}{|c|c|c|c|c|c|c|c|c|c|c|}
\hline 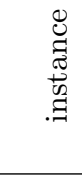 & 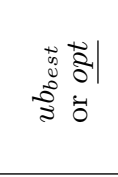 & 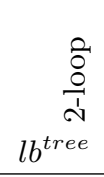 & $\begin{array}{r}\text { ô } \\
\frac{0}{1} \\
\text { oे } \\
l b^{\text {tree }}\end{array}$ & 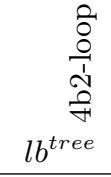 & $l b^{\text {tree }}$ & $\begin{array}{r}n g 4 \\
l b^{\text {tree }}\end{array}$ & $\begin{array}{r}n g 5 \\
l b^{\text {tree }}\end{array}$ & $\begin{array}{r}n g 6 \\
l b^{\text {tree }}\end{array}$ & $\begin{array}{r}n g 7 \\
l b^{\text {tree }}\end{array}$ & $l b_{\text {own }}^{\text {best }}$ \\
\hline e1-a & 3548 & OPT & OPT & OPT & OPT & OPT & OPT & OPT & OPT & OPT \\
\hline e1-b & $\overline{4498}$ & OPT & OPT & OPT & OPT & OPT & OPT & OPT & OPT & OPT \\
\hline e1-c & $\underline{5595}$ & 5545 & 5551 & 5555 & 5554 & 5560 & 5571 & 5570 & 5572 & 5572 \\
\hline$e 2-a$ & $\underline{5018}$ & OPT & OPT & OPT & OPT & OPT & 5018 & 5018 & 5012 & OPT \\
\hline$e 2-b$ & $\underline{6317}$ & 6301 & 6301 & 6306 & 6305 & 6308 & 6311 & OPT & OPT & OPT \\
\hline$e 2-c$ & $\underline{8335}$ & 8242 & 8269 & 8303 & 8302 & 8300 & 8304 & 8315 & 8317 & 8317 \\
\hline e3-a & $\underline{5898}$ & OPT & OPT & OPT & OPT & OPT & OPT & OPT & 5898 & OPT \\
\hline e3-b & $\overline{7775}$ & 7730 & 7735 & 7732 & 7733 & 7734 & 7741 & 7741 & 7740 & 7741 \\
\hline e3-c & 10292 & 10191 & 10220 & 10226 & 10225 & 10226 & 10228 & 10228 & 10229 & 10229 \\
\hline e4-a & 6444 & 6408 & 6405 & 6399 & 6399 & 6398 & 6399 & 6399 & 6398 & 6408 \\
\hline e4-b & 8961 & 8892 & 8899 & 8900 & 8897 & 8905 & 8908 & 8913 & 8910 & 8913 \\
\hline e4-c & 11529 & 11456 & 11488 & 11501 & 11499 & 11499 & 11500 & 11501 & 11501 & 11501 \\
\hline s1-a & $\underline{5018}$ & $\mathrm{OPT}$ & OPT & OPT & OPT & 5018 & 5018 & 5018 & 5015 & OPT \\
\hline s1-b & $\underline{6388}$ & 6386 & OPT & OPT & OPT & 6384 & 6384 & 6385 & 6383 & OPT \\
\hline s1-c & $\underline{8518}$ & 8440 & 8476 & 8500 & 8499 & 8501 & 8504 & 8509 & 8507 & 8509 \\
\hline $\mathrm{s} 2-\mathrm{a}$ & $\overline{9884}$ & 9805 & 9806 & 9804 & 9803 & 9807 & 9806 & 9806 & 9808 & 9808 \\
\hline$s 2-b$ & 13100 & 12970 & 12978 & 12982 & 12980 & 12991 & 12991 & 12994 & 12994 & 12994 \\
\hline$s 2-c$ & 16425 & 16351 & 16377 & 16380 & 16379 & 16393 & 16392 & 16393 & 16393 & 16393 \\
\hline s3-a & $\overline{10220}$ & 10160 & 10154 & 10150 & 10149 & 10153 & 10153 & 10154 & 10152 & 10160 \\
\hline s3-b & 13682 & 13630 & 13629 & 13627 & 13625 & 13637 & 13640 & 13644 & 13640 & 13644 \\
\hline s3-c & 17188 & 17096 & 17122 & 17125 & 17123 & 17138 & 17143 & 17142 & 17141 & 17143 \\
\hline s4-a & $\overline{12268}$ & 12149 & 12147 & 12142 & 12141 & 12150 & 12151 & 12151 & 12150 & 12151 \\
\hline s4-b & 16283 & 16104 & 16106 & 16105 & 16104 & 16113 & 16111 & 16111 & 16108 & 16113 \\
\hline$s 4-c$ & 20481 & 20374 & 20397 & 20406 & 20405 & 20418 & 20420 & 20422 & 20423 & 20423 \\
\hline & best & 7 & 6 & 7 & & 6 & 6 & 12 & 11 & \\
\hline
\end{tabular}


Table 10: Integer Results for bmcv Instances, Subsets C and E

\begin{tabular}{|c|c|c|c|c|c|c|c|c|c|}
\hline 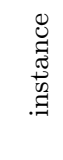 & 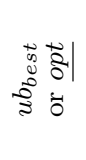 & 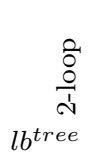 & 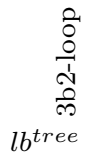 & 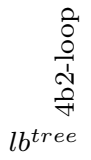 & 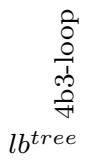 & lb & $l b^{\text {tree }}$ & $l b^{\text {tree }}$ & 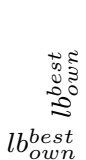 \\
\hline $\mathrm{C01}$ & 4150 & 4144 & 4140 & 4140 & 4138 & 4143 & 4145 & 4144 & 4145 \\
\hline $\mathrm{CO} 2$ & 3135 & OPT & OPT & OPT & OPT & OPT & OPT & OPT & $\mathrm{OPT}$ \\
\hline $\mathrm{CO} 3$ & $\underline{2575}$ & OPT & OPT & OPT & OPT & OPT & OPT & OPT & OPT \\
\hline $\mathrm{CO} 4$ & 3510 & OPT & OPT & OPT & OPT & OPT & OPT & OPT & OPT \\
\hline $\mathrm{CO5}$ & 5365 & OPT & OPT & OPT & OPT & OPT & OPT & OPT & OPT \\
\hline $\mathrm{CO} 6$ & 2535 & OPT & OPT & OPT & OPT & OPT & OPT & OPT & OPT \\
\hline $\mathrm{CO} 7$ & 4075 & OPT & OPT & OPT & OPT & OPT & OPT & OPT & OPT \\
\hline $\mathrm{C08}$ & 4090 & OPT & OPT & OPT & OPT & OPT & OPT & OPT & OPT \\
\hline $\mathrm{CO9}$ & 5260 & 5244 & 5242 & 5242 & 5241 & 5245 & 5245 & 5245 & 5245 \\
\hline C10 & $\underline{4700}$ & OPT & OPT & OPT & OPT & OPT & OPT & OPT & OPT \\
\hline C11 & 4635 & 4608 & 4608 & 4607 & 4604 & 4609 & 4611 & 4609 & 4611 \\
\hline C12 & 4240 & 4234 & 4231 & 4226 & 4225 & 4233 & 4232 & 4232 & 4234 \\
\hline C13 & $\underline{2955}$ & OPT & OPT & OPT & OPT & OPT & OPT & OPT & OPT \\
\hline C14 & $\underline{4030}$ & 4010 & 4021 & 4024 & 4019 & $\mathrm{OPT}$ & OPT & OPT & $\mathrm{OPT}$ \\
\hline C15 & $\overline{4940}$ & 4918 & 4915 & 4916 & 4914 & 4918 & 4918 & 4918 & 4918 \\
\hline C16 & $\underline{1475}$ & OPT & OPT & $\mathrm{OPT}$ & $\mathrm{OPT}$ & $\mathrm{OPT}$ & $\mathrm{OPT}$ & $\mathrm{OPT}$ & OPT \\
\hline C17 & $\overline{3555}$ & OPT & OPT & OPT & OPT & OPT & OPT & OPT & OPT \\
\hline C18 & $\overline{5620}$ & 5570 & 5568 & 5563 & 5562 & 5564 & 5562 & 5562 & 5570 \\
\hline C19 & $\underline{3115}$ & OPT & OPT & OPT & OPT & OPT & OPT & OPT & OPT \\
\hline $\mathrm{C} 2 \mathrm{O}$ & 2120 & OPT & OPT & OPT & OPT & OPT & OPT & OPT & $\mathrm{OPT}$ \\
\hline $\mathrm{C} 21$ & $\overline{3970}$ & OPT & OPT & OPT & OPT & OPT & OPT & OPT & OPT \\
\hline $\mathrm{C} 22$ & $\overline{2245}$ & OPT & OPT & OPT & OPT & OPT & OPT & OPT & OPT \\
\hline $\mathrm{C} 23$ & $\overline{4085}$ & 4073 & 4072 & 4069 & 4070 & 4073 & 4068 & 4058 & 4073 \\
\hline $\mathrm{C} 24$ & $\underline{3400}$ & OPT & OPT & OPT & OPT & OPT & OPT & OPT & OPT \\
\hline $\mathrm{C} 25$ & $\underline{2310}$ & OPT & OPT & OPT & OPT & OPT & OPT & OPT & OPT \\
\hline \multicolumn{2}{|c|}{ Num $l b_{\text {own }}^{\text {best }}$} & 21 & 17 & 17 & 17 & 21 & 22 & 20 & \\
\hline E01 & 4910 & 4898 & 4896 & 4896 & 4893 & 4898 & 4897 & 4897 & 4898 \\
\hline E02 & 3990 & 3971 & 3985 & OPT & OPT & OPT & OPT & OPT & OPT \\
\hline E03 & $\overline{2015}$ & OPT & OPT & OPT & OPT & OPT & OPT & OPT & OPT \\
\hline E04 & $\overline{4155}$ & OPT & OPT & OPT & OPT & OPT & OPT & OPT & OPT \\
\hline E05 & $\overline{4585}$ & OPT & OPT & OPT & OPT & OPT & OPT & OPT & OPT \\
\hline E06 & $\underline{2055}$ & OPT & OPT & OPT & OPT & OPT & OPT & OPT & $\mathrm{OPT}$ \\
\hline E07 & 4155 & 4137 & 4149 & OPT & OPT & OPT & OPT & OPT & OPT \\
\hline E08 & $\underline{4710}$ & OPT & OPT & OPT & OPT & $\mathrm{OPT}$ & $\mathrm{OPT}$ & $\mathrm{OPT}$ & OPT \\
\hline E09 & 5820 & 5802 & 5800 & 5798 & 5797 & 5802 & 5802 & 5802 & 5802 \\
\hline E10 & $\underline{3605}$ & OPT & OPT & OPT & OPT & $\mathrm{OPT}$ & OPT & OPT & OPT \\
\hline E11 & 4650 & 4650 & OPT & 4650 & 4650 & 4650 & OPT & OPT & OPT \\
\hline E12 & $\underline{4180}$ & 4167 & 4169 & 4170 & 4166 & 4178 & 4177 & 4179 & 4179 \\
\hline E13 & $\overline{3345}$ & OPT & OPT & OPT & OPT & OPT & OPT & OPT & OPT \\
\hline E14 & $\underline{4115}$ & 4108 & OPT & OPT & OPT & OPT & 4111 & OPT & $\mathrm{OPT}$ \\
\hline E15 & $\overline{4205}$ & 4199 & 4196 & 4194 & 4192 & 4197 & 4192 & 4193 & 4199 \\
\hline E16 & 3775 & OPT & OPT & OPT & OPT & OPT & OPT & OPT & OPT \\
\hline E17 & $\overline{2740}$ & OPT & OPT & OPT & OPT & OPT & OPT & OPT & OPT \\
\hline E18 & 3835 & 3825 & 3825 & 3825 & 3825 & 3826 & 3831 & 3832 & 3832 \\
\hline E19 & $\underline{3235}$ & OPT & OPT & OPT & 3235 & 3235 & 3235 & 3235 & OPT \\
\hline E20 & $\underline{2825}$ & 2815 & 2820 & OPT & OPT & OPT & OPT & OPT & OPT \\
\hline E21 & $\underline{3730}$ & 3730 & 3730 & 3730 & 3730 & 3730 & OPT & OPT & OPT \\
\hline E22 & $\overline{2470}$ & OPT & OPT & OPT & OPT & OPT & OPT & OPT & OPT \\
\hline E23 & $\overline{3710}$ & 3704 & 3703 & 3699 & 3697 & 3707 & 3704 & 3701 & 3707 \\
\hline E24 & 4020 & OPT & 4020 & OPT & 4020 & OPT & OPT & OPT & OPT \\
\hline E25 & $\underline{1615}$ & OPT & OPT & OPT & OPT & OPT & OPT & OPT & OPT \\
\hline \multicolumn{2}{|c|}{ Num $l b_{\text {own }}^{\text {best }}$} & 16 & 14 & 17 & 15 & 19 & 18 & 21 & \\
\hline
\end{tabular}


Table 11: Integer Results for bmcv Instances, Subsets D and F

\begin{tabular}{|c|c|c|c|c|c|c|c|c|c|}
\hline 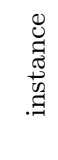 & 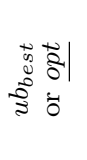 & 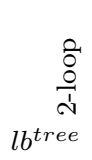 & $\begin{array}{r}\stackrel{0}{0} \\
\stackrel{0}{1} \\
\text { N } \\
\text { ले } \\
l b^{\text {tree }}\end{array}$ & 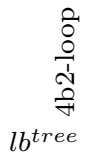 & 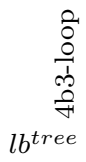 & $l b^{\text {tree }}$ & $l b^{\text {tree }}$ & $l b^{\text {tree }} \begin{array}{r}\text { bे } \\
\text { a }\end{array}$ & 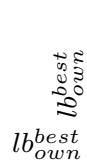 \\
\hline D01 & $\underline{3215}$ & OPT & OPT & OPT & 3215 & OPT & OPT & OPT & OPT \\
\hline D02 & 2520 & OPT & OPT & OPT & OPT & OPT & OPT & OPT & OPT \\
\hline D03 & $\underline{2065}$ & OPT & $\mathrm{OPT}$ & OPT & OPT & $\mathrm{OPT}$ & OPT & OPT & OPT \\
\hline D04 & 2785 & OPT & OPT & OPT & OPT & OPT & 2785 & 2785 & OPT \\
\hline D05 & 3935 & OPT & OPT & OPT & OPT & OPT & OPT & OPT & OPT \\
\hline D06 & $\overline{2125}$ & OPT & OPT & OPT & OPT & OPT & OPT & OPT & OPT \\
\hline D07 & $\underline{3115}$ & 3108 & 3102 & 3098 & 3092 & 3098 & 3090 & 3082 & 3108 \\
\hline D08 & $\overline{3045}$ & OPT & 3041 & 3027 & 3022 & 3030 & 3027 & 3004 & OPT \\
\hline D09 & 4120 & OPT & OPT & OPT & OPT & OPT & OPT & 4120 & OPT \\
\hline D10 & $\underline{3340}$ & OPT & OPT & OPT & OPT & OPT & OPT & OPT & OPT \\
\hline D11 & 3745 & 3745 & OPT & OPT & 3745 & 3745 & 3745 & 3745 & OPT \\
\hline D12 & $\underline{3310}$ & OPT & OPT & OPT & OPT & $\mathrm{OPT}$ & $\mathrm{OPT}$ & OPT & OPT \\
\hline D13 & $\underline{2535}$ & OPT & OPT & OPT & OPT & OPT & OPT & OPT & OPT \\
\hline D14 & $\underline{3280}$ & 3280 & OPT & OPT & OPT & OPT & OPT & OPT & OPT \\
\hline D15 & $\overline{3990}$ & OPT & OPT & - & 3990 & 3990 & 3990 & 3990 & $\mathrm{OPT}$ \\
\hline D16 & $\underline{1060}$ & OPT & $\mathrm{OPT}$ & OPT & OPT & $\mathrm{OPT}$ & $\mathrm{OPT}$ & 1060 & OPT \\
\hline D17 & $\overline{2620}$ & OPT & OPT & OPT & OPT & OPT & OPT & OPT & OPT \\
\hline D18 & $\underline{4165}$ & OPT & - & 4165 & - & 4165 & 4165 & 4165 & $\mathrm{OPT}$ \\
\hline D19 & $\overline{2400}$ & OPT & OPT & OPT & OPT & 2376 & 2373 & 2373 & OPT \\
\hline D20 & 1870 & OPT & OPT & OPT & OPT & 1870 & 1870 & 1870 & OPT \\
\hline D21 & $\overline{3050}$ & 3005 & 2988 & 2982 & 2980 & 2983 & 2981 & 2981 & 3005 \\
\hline D22 & $\underline{1865}$ & $\mathrm{OPT}$ & OPT & OPT & OPT & $\mathrm{OPT}$ & OPT & OPT & OPT \\
\hline D23 & $\overline{3130}$ & 3126 & 3114 & 3111 & 3111 & 3115 & 3113 & 3113 & 3126 \\
\hline D24 & 2710 & 2704 & 2691 & 2679 & 2669 & 2669 & 2666 & 2666 & 2704 \\
\hline D25 & $\underline{1815}$ & OPT & OPT & OPT & OPT & OPT & $\mathrm{OPT}$ & OPT & OPT \\
\hline \multicolumn{2}{|c|}{ Num $l b_{\text {own }}^{\text {best }}$} & 23 & 19 & 18 & 16 & 15 & 14 & 12 & \\
\hline F01 & $\underline{4040}$ & OPT & OPT & OPT & OPT & OPT & OPT & 4040 & OPT \\
\hline F02 & $\overline{3300}$ & OPT & OPT & OPT & OPT & OPT & OPT & OPT & OPT \\
\hline F03 & $\overline{1665}$ & OPT & OPT & OPT & OPT & OPT & OPT & OPT & OPT \\
\hline F04 & $\overline{3485}$ & OPT & OPT & OPT & 3485 & 3483 & 3477 & 3476 & OPT \\
\hline F05 & $\overline{3605}$ & OPT & OPT & OPT & OPT & OPT & OPT & OPT & OPT \\
\hline F06 & $\underline{1875}$ & OPT & OPT & OPT & OPT & $\mathrm{OPT}$ & OPT & OPT & OPT \\
\hline F07 & $\underline{3335}$ & OPT & OPT & OPT & OPT & OPT & OPT & OPT & OPT \\
\hline F08 & $\underline{3705}$ & OPT & OPT & OPT & OPT & OPT & OPT & OPT & OPT \\
\hline F09 & $\underline{4730}$ & OPT & OPT & 4730 & 4730 & 4730 & 4730 & 4730 & OPT \\
\hline F10 & $\underline{2925}$ & OPT & OPT & OPT & OPT & OPT & $\mathrm{OPT}$ & OPT & OPT \\
\hline F11 & $\underline{3835}$ & OPT & $\mathrm{OPT}$ & OPT & OPT & 3835 & 3835 & 3835 & OPT \\
\hline F12 & $\underline{3395}$ & OPT & 3395 & 3392 & 3392 & 3392 & 3390 & 3390 & OPT \\
\hline F13 & $\overline{2855}$ & OPT & OPT & OPT & OPT & OPT & OPT & OPT & OPT \\
\hline F14 & $\underline{3330}$ & OPT & $\mathrm{OPT}$ & $\mathrm{OPT}$ & OPT & OPT & OPT & OPT & OPT \\
\hline F15 & $\overline{3560}$ & OPT & 3560 & 3560 & OPT & 3560 & 3560 & 3560 & OPT \\
\hline F16 & $\underline{2725}$ & OPT & $\mathrm{OPT}$ & OPT & OPT & OPT & OPT & OPT & OPT \\
\hline F17 & $\overline{2055}$ & OPT & OPT & OPT & OPT & OPT & OPT & OPT & OPT \\
\hline F18 & $\overline{3075}$ & 3065 & 3065 & 3065 & 3065 & 3062 & 3062 & 3062 & 3065 \\
\hline F19 & 2525 & 2515 & 2515 & 2514 & 2511 & 2489 & 2489 & 2488 & 2515 \\
\hline F20 & $\underline{2445}$ & OPT & OPT & OPT & OPT & OPT & $\mathrm{OPT}$ & OPT & OPT \\
\hline F21 & $\underline{2930}$ & OPT & OPT & OPT & 2930 & OPT & 2930 & OPT & OPT \\
\hline F22 & $\underline{2075}$ & OPT & OPT & OPT & OPT & OPT & OPT & OPT & OPT \\
\hline F23 & $\overline{3005}$ & 3003 & 2998 & 2994 & 2996 & 2989 & 2989 & 2989 & 3003 \\
\hline F24 & $\underline{3210}$ & OPT & OPT & OPT & OPT & 3210 & 3210 & 3210 & OPT \\
\hline F25 & 1390 & $\mathrm{OPT}$ & OPT & OPT & OPT & OPT & $\mathrm{OPT}$ & OPT & OPT \\
\hline
\end{tabular}


Table 12: Integer Results for Large-Scale egl Instances

\begin{tabular}{|c|c|c|c|}
\hline 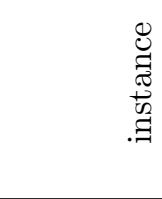 & $u b_{\text {best }}$ & 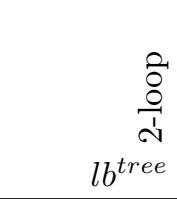 & 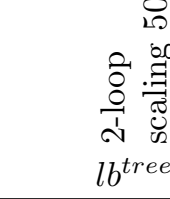 \\
\hline egl-g1-a & $1,004,864$ & 974,383 & 976,907 \\
\hline egl-g1-b & $1,129,937$ & $1,092,760$ & $1,093,884$ \\
\hline egl-g1-c & $1,262,888$ & $1,211,590$ & $1,212,151$ \\
\hline egl-g1-d & $1,398,958$ & $1,341,370$ & $1,341,918$ \\
\hline egl-g1-e & $1,543,804$ & $1,481,500$ & $1,482,176$ \\
\hline egl-g2-a & $1,115,339$ & $1,069,536$ & $1,067,262$ \\
\hline egl-g2-b & $1,226,645$ & $1,184,230$ & $1,185,221$ \\
\hline egl-g2-c & $1,371,004$ & $1,308,960$ & $1,311,339$ \\
\hline egl-g2-d & $1,509,990$ & $1,445,870$ & $1,446,680$ \\
\hline egl-g2-e & $1,659,217$ & $1,580,030$ & $1,581,459$ \\
\hline
\end{tabular}

Finally, the Table 13 presents the integer results for strong branching using the standard egl instances. The meaning of the table entries are as follows:

instance name of the instance

$u b_{\text {best }}$ or opt the best known upper bound (not underlined) or the optimum (underlined)

$l b^{\text {tree }}-$ lower bound provided by the branch-and-price algorithm within the time limit of 4 hours (rounded up to the next integer)

'OPT' indicates that the instance is solved to proven optimality within 4 hours $l b^{\text {tree }}=o p t$ indicates that the gap was closed, but no integer optimal solution was computed within the time limit

$u b_{\text {own }}^{\text {best }} \quad$ best lower bound computed in this analysis 


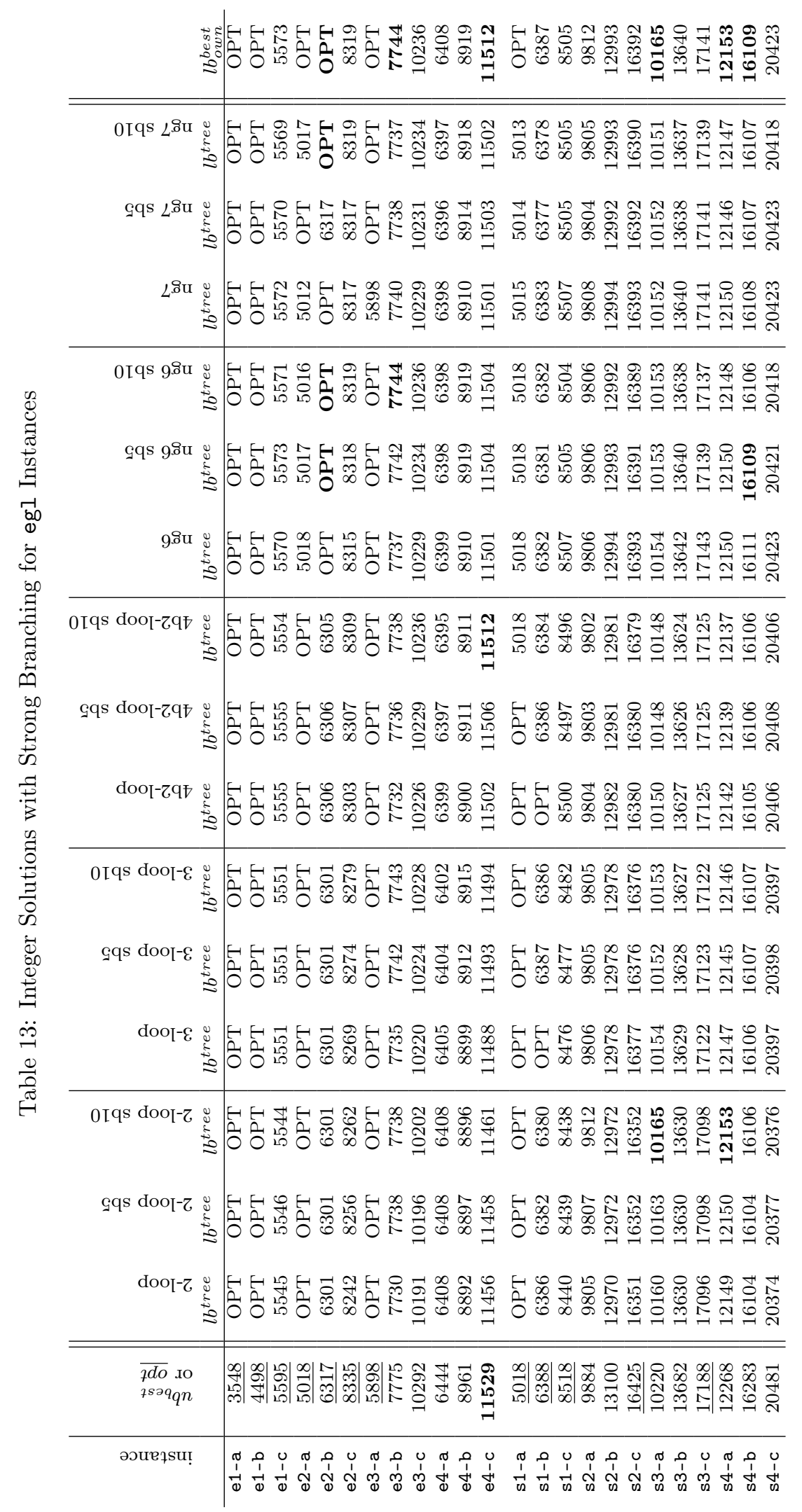




\section{Best Known Lower and Upper Bounds}

The Tables 14,16 list the best known lower and upper bounds for the standard and large-scale egl instances and the bmcv instances. The meaning of the table entries are as follows:

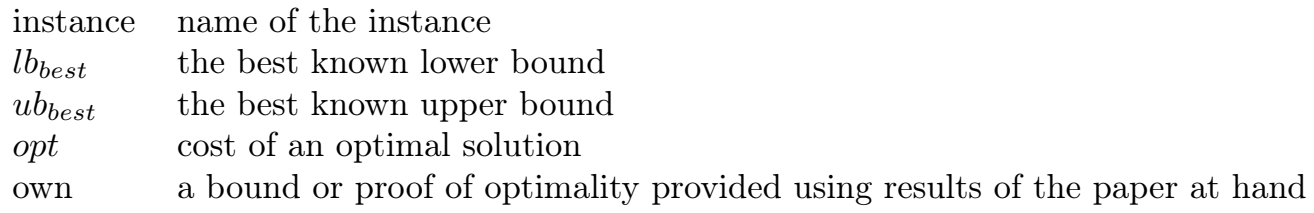

Note: if an instance is solved to optimality, we do not give a lower bound.

At the time of writing this paper, twelve of the standard and all twelve large-scale egl instances remain unsolved. For the bmcv benchmark set, seven C, two D, three E, and two F instances are open. 


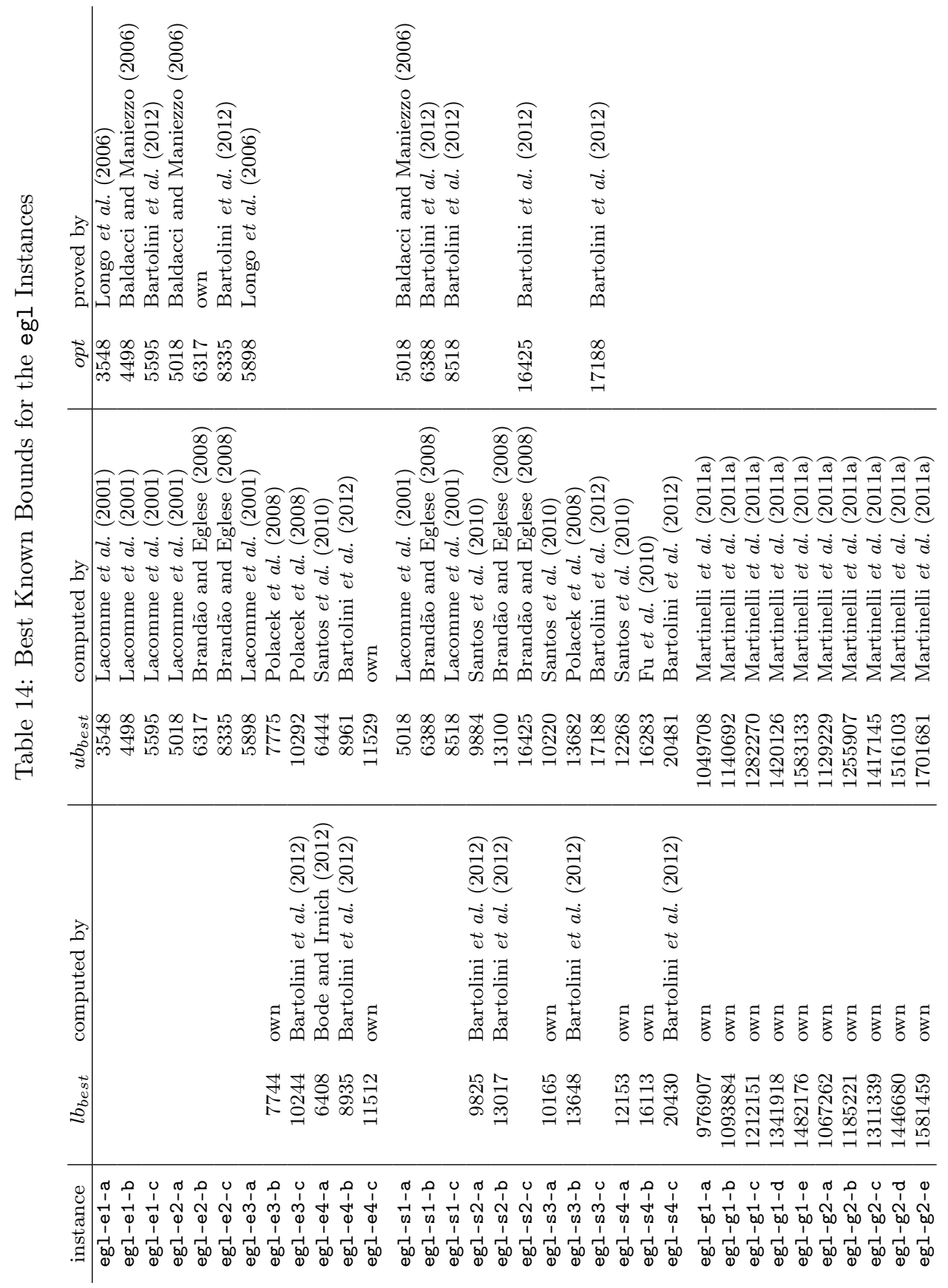


Table 15: Best Known Bounds for the bmcv Instances, Subsets C and E

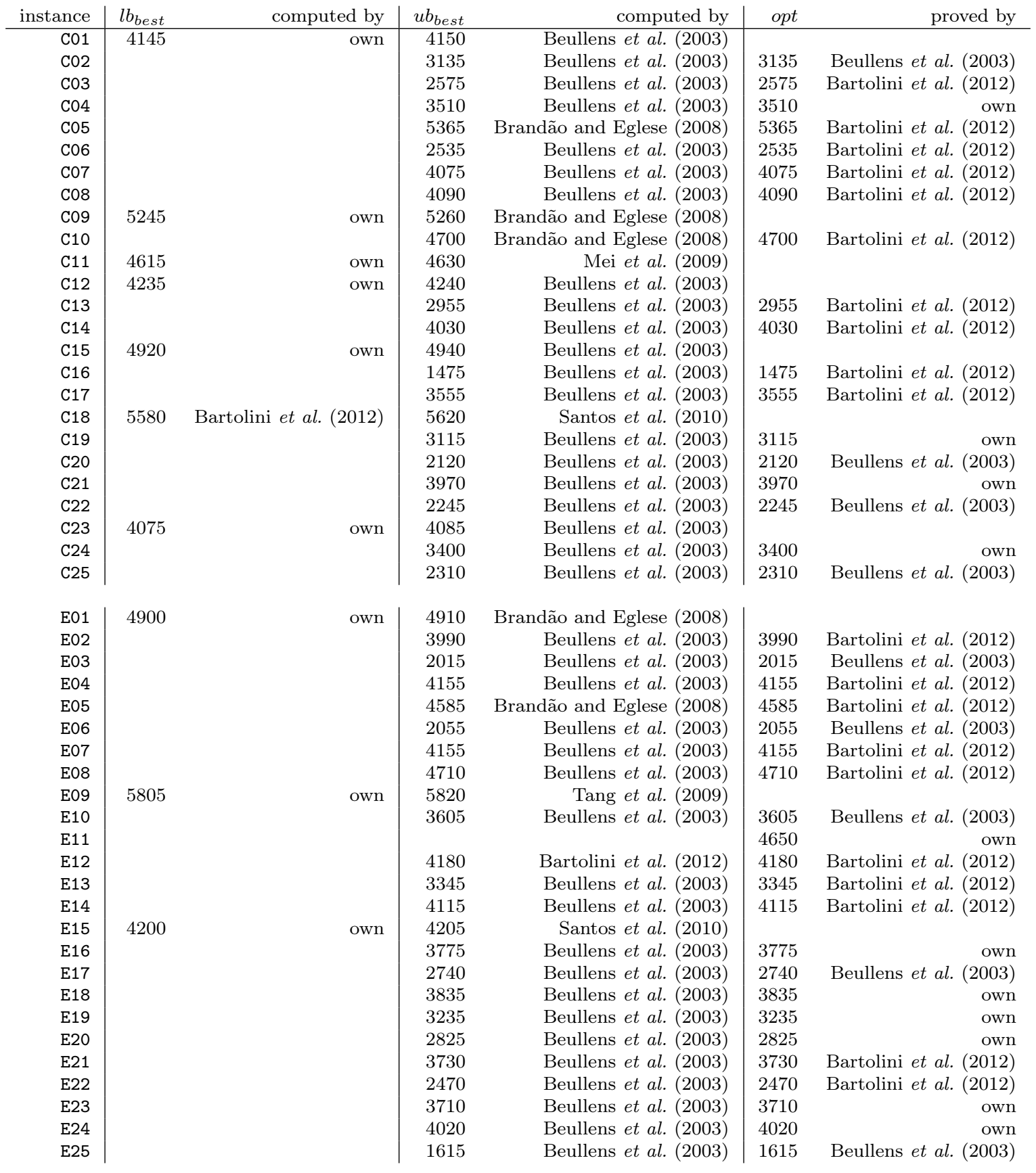


Table 16: Best Known Bounds for the bmcv Instances, Subsets D and F

\begin{tabular}{|c|c|c|c|c|c|c|c|}
\hline instance & $l b_{\text {best }}$ & computed by & $u b_{\text {best }}$ & compu & ited by & opt & proved by \\
\hline D01 & & & 3215 & Beullens et al. & $(2003)$ & 3215 & Beullens et al. \\
\hline D02 & & & 2520 & Beullens et al. & $(2003)$ & 2520 & Beullens et al. (2003) \\
\hline D03 & & & 2065 & Beullens et al. & (2003) & 2065 & Beullens et al. (2003) \\
\hline D04 & & & 2785 & Beullens et al. & 2003 & 2785 & Beullens et al. \\
\hline D05 & & & 3935 & Beullens et al. & $(2003)$ & 3935 & Beullens et al. \\
\hline D06 & & & 2125 & Beullens et al. & 2003 & 2125 & Beullens et al. 2003 \\
\hline D07 & & & 3115 & Beullens et al. & $(2003)$ & 3115 & Bartolini et al. \\
\hline D08 & & & 3045 & Beullens et al. & $(2003)$ & 3045 & own \\
\hline D09 & & & 4120 & Beullens et al. & $(2003)$ & 4120 & Beullens et al. (2003) \\
\hline D10 & & & 3340 & Beullens et al. & $(2003)$ & 3340 & Bartolini et al. \\
\hline D11 & & & 3745 & T'ang et al. & $(2009)$ & 3745 & Beullens et al. (2003) \\
\hline D12 & & & 3310 & Beullens et al. & 2003 & 3310 & Beullens et al. \\
\hline D13 & & & 2535 & Beullens et al. & 2003 & 2535 & Beullens et al. $(\overline{2003})$ \\
\hline D14 & & & 3280 & Beullens et al. & $(2003)$ & 3280 & own \\
\hline D15 & & & 3990 & Beullens et al. & 2003 & 3990 & Beullens et al. 2003 \\
\hline D16 & & & 1060 & Beullens et al. & $(2003)$ & 1060 & Beullens et al. (2003) \\
\hline D17 & & & 2620 & Beullens et al. & $(2003)$ & 2620 & Beullens et al. (2003) \\
\hline D18 & & & 4165 & Beullens et al. & $(2003)$ & 4165 & Beullens et al. \\
\hline D19 & & & 2400 & Beullens et al. & 2003 & 2400 & own \\
\hline D20 & & & 1870 & Beullens et al. & 2003 & 1870 & Beullens et al. (2003) \\
\hline D21 & 3005 & own & 3050 & Beullens et al. & 2003 & & \\
\hline D22 & & & 1865 & Beullens et al. & 2003 & 1865 & Beullens et al. (2003) \\
\hline D23 & & & 3130 & Beullens et al. & 2003 & 3130 & own \\
\hline D24 & 2705 & own & 2710 & Beullens et al. & 2003 & & \\
\hline D25 & & & 1815 & Beullens et al. & $(2003)$ & 1815 & Beullens et al. (2003) \\
\hline F01 & & & 4040 & Beullens et al. & 2003 & 4040 & Beullens et al. (2003) \\
\hline F02 & & & 3300 & Beullens et al. & 2003 & 3300 & Beullens et al. \\
\hline F03 & & & 1665 & Beullens et al. & 2003 & 1665 & Beullens et al. $(\overline{2003})$ \\
\hline F04 & & & 3485 & Beullens et al. & $(2003)$ & 3485 & own \\
\hline F05 & & & 3605 & Beullens et al. & (2003) & 3605 & Beullens et al. (2003) \\
\hline F06 & & & 1875 & Beullens et al. & $(2003)$ & 1875 & Beullens et al. (2003) \\
\hline F07 & & & 3335 & Beullens et al. & $(2003)$ & 3335 & 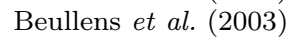 \\
\hline F08 & & & 3705 & Beullens et al. & $\overline{2003}$ & 3705 & own \\
\hline F09 & & & 4730 & Beullens et al. & 2003 & 4730 & Beullens et al. 2003) \\
\hline F10 & & & 2925 & Beullens et al. & 2003 & 2925 & Beullens et al. (2003) \\
\hline $\mathrm{F} 11$ & & & 3835 & Beullens et al. & 2003 & 3835 & Beullens et al. (2003) \\
\hline $\mathrm{F} 12$ & & & 3395 & Beullens et al. & $(2003)$ & 3395 & own \\
\hline $\mathrm{F} 13$ & & & 2855 & Beullens et al. & $(2003)$ & 2855 & Beullens et al. (2003) \\
\hline F14 & & & 3330 & Beullens et al. & 2003 & 3330 & Beullens et al. (2003) \\
\hline F15 & & & 3560 & Beullens et al. & 2003 & 3560 & Beullens et al. \\
\hline F16 & & & 2725 & Beullens et al. & 2003 & 2725 & Beullens et al. $(2003)$ \\
\hline $\mathrm{F} 17$ & & & 2055 & Beullens et al. & 2003 & 2055 & 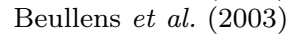 \\
\hline F18 & 3065 & Bartolini et al. (2012) & 3075 & Beullens et al. & 2003 & & \\
\hline F19 & 2515 & own & 2525 & Beullens et al. & 2003 & & \\
\hline F20 & & & 2445 & Beullens et al. & 2003 & 2445 & Beullens et al. (2003) \\
\hline F21 & & & 2930 & Beullens et al. & 2003 & 2930 & Beullens et al. \\
\hline F22 & & & 2075 & Beullens et al. & 2003 & 2075 & Beullens et al. $(\overline{2003})$ \\
\hline F23 & & & 3005 & Beullens et al. & 2003 & 3005 & own \\
\hline F24 & & & 3210 & Beullens et al. & 2003 & 3210 & Beullens et al. (2003) \\
\hline F25 & & & 1390 & Beullens et al. & 2003 & 1390 & Beullens et al. (2003) \\
\hline
\end{tabular}




\section{Integer Solutions}

In this section, new integer solutions are given. Note that in the following ' $=$ ' indicates a service and ' - ' a deadheading. The terms $u b$ and opt show the cost of the presented solution. 'load' is the demand served by the respective route.

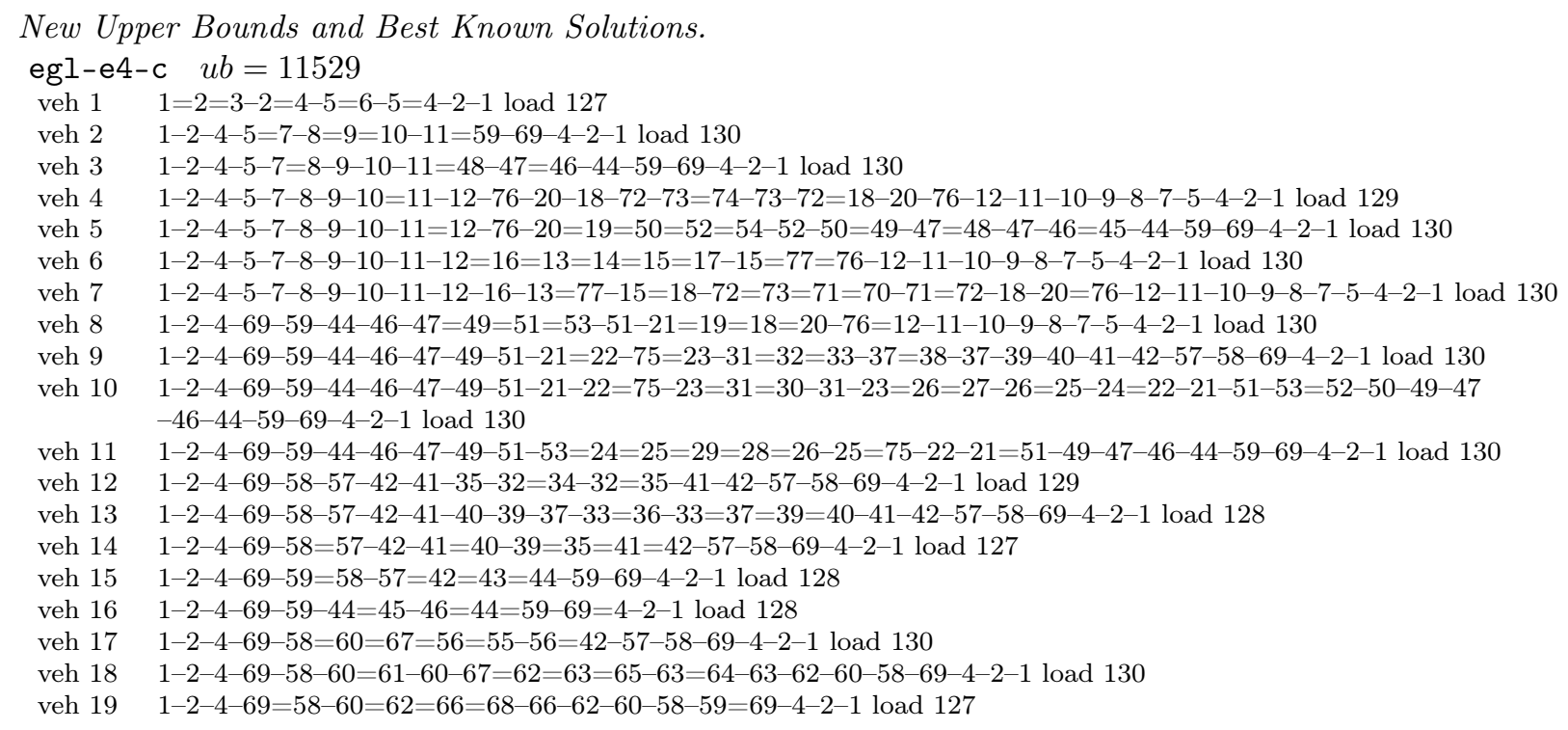

\section{New Optimal Solutions.}

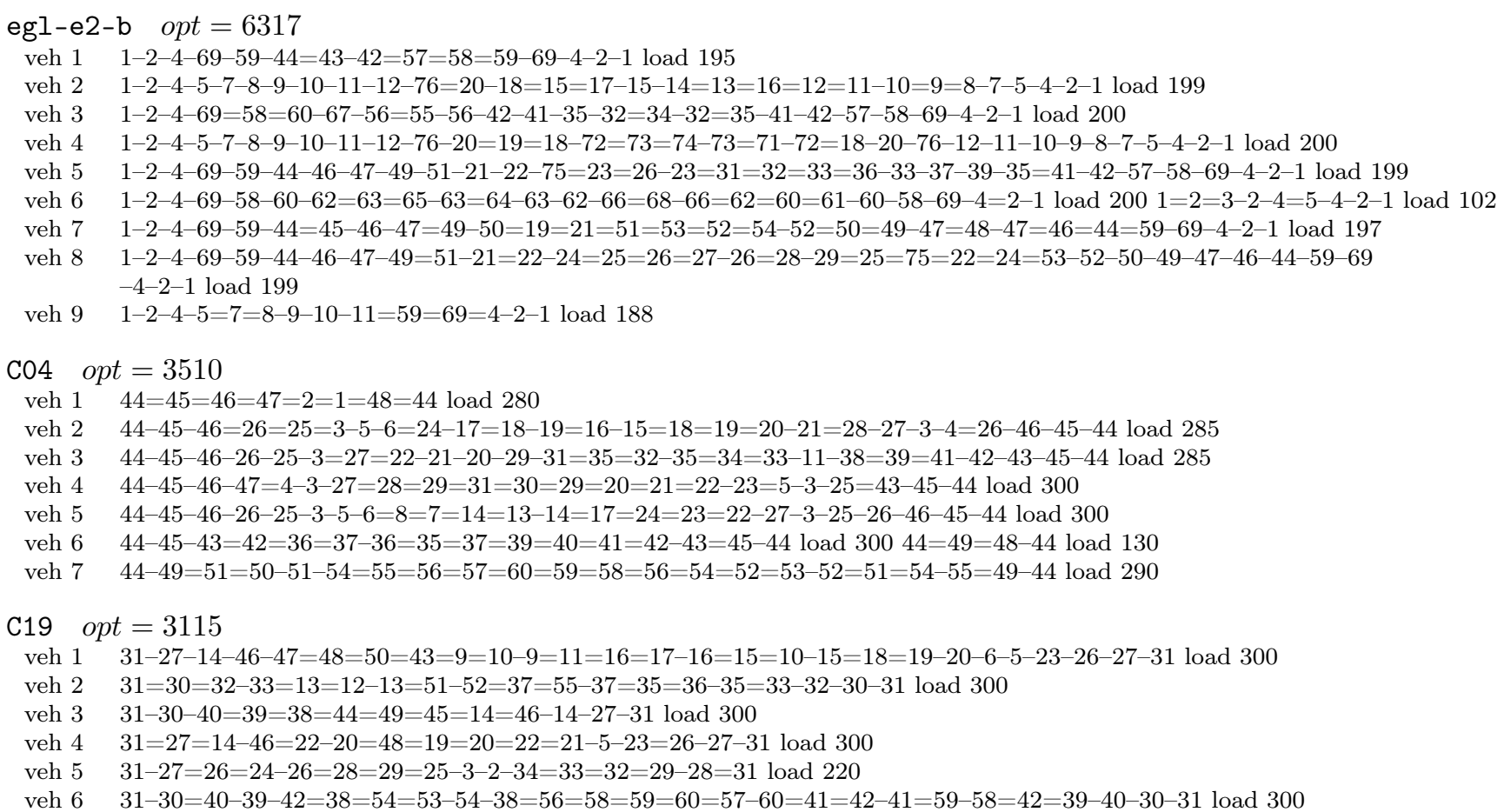


C21 opt $=3970$

veh $134-31-33-38-40-43=44-49=2=1=7=10=9=8=47-45=43-40-38-33-31-34$ load 300

veh $234-35-37-19=21=53=42-41=51=50=3=52=4-52=51-50=49-44=45-43=40=38-33-31-34$ load 300

veh $3 \quad 34-35-37-19=20=22=60=59=58=57=11=12-11=13-15-16=17=18-19-37-35-34$ load 290

veh $4 \quad 34-31-33-38-40=41=42=21=20=17=15=14=13=15=16=22=21-19-37-35-34$ load 280

veh $5 \quad 34-35=37=19=18=23=37=36=35-34$ load 265

veh $6 \quad 34-31=23=24-23=25=26=28-26=27=25-27=29-27=30=32-30=31-34$ load 300

veh $7 \quad 34=31=32=33=38=39-38=36-35=34$ load 210

veh $8 \quad 34-31=33-38-40-43-44=49=47=48-47=45=46-45-43-40-38-33-31-34$ load 300

C24 opt $=3400$

veh $149-47-48-42-52=51-35-30=3-4=53=55-54=60=56-1=2=6=21-6=5=52=42-48-47-49$ load 300

veh $249-47-48-13-12=5=3=4=2=54=55=28-62=61=59=58=57=60=61=55-53=29=3-30-35-36-31=43=41-39-47-49$ load 300

veh $3 \quad 49-7=10=11=12=13=48=42=41=40=44=43=42-48=47=49$ load 285

veh $4 \quad 49-47=39=46=45=66=68=8-9=15=50=14-50=9=8=7=49$ load 295

veh $5 \quad 49-7=16=17=18=20=19=11=13=10-7-49$ load 300

veh $6 \quad 49-47-39=40-44-32-33-38=37=34-37=26=27=25=24=76-70-75-74-63=38=33-32-44-40-41=39-47-49$ load 295

veh $7 \quad 49-47=46=44=32=69-65=38=64-65=69=67=45-46-47-49$ load 265

D08 opt $=3045$

veh $1 \quad 45-46=35=36=33=32=1-32=31=30-31=12-11=34=35=37-43=44-45$ load 575

veh $245-46-48-49-51=50=60=61=2=40=41-42=38=5=4=3=39=41-44=45$ load 600

veh $345-46-48=47=16-18=54=29=19=18-16=17=15=10=9=34=33-36=37=43=42=41=44-45$ load 600

veh $4 \quad 45=46=48=49=47=55=53-55=56-57=64=65=66=29-54=56=57=62=63=58=52=59-52=53=51=49-48-46-45$ load 585

D14 $z=y 3280$

veh $1 \quad 34-35-37=47-48=36=39=40-39=38=33-32=22-21-1=2-1=14-15=16-17-35-34$ load 600

veh $234=35-17=16=20=19=18=51=52=4=1=21=22=23=3=2=5=4=53-4=19-18=17-35-34$ load 600

veh $3 \quad 34-35=37=36=33=32=31-11-25=24-26=28=30-28=29-12-27=26=24=23-22-21=15=14=20-16-17=35-34$ load 570

veh $4 \quad 34=33-38=42=45=46-45=44-10-43=42=40=41=49=50=41=39-38-33-34$ load 580

D19 $z=y 2400$

veh $131-28=26=24-26=23-5-21=22-20=48=19-48=47-48=50=43=9=10-9=11=16=17-16=15=10-15=18=19=20=22=46=14$ $-27=31$ load 590

veh $2 \quad 31-28-29=32=30-32=33=13=12-13=51-52=37-55-57-60=41-42=58-59=60=57-55=37=35=36-35=33=34-2-3-25=29=28$ $=31$ load 535

veh $331=30=40=39=42-39=38=54=53-54-38=56=58=59=41=42=38=44=49=45=14=27=26-27-31$ load 595

E11 opt $=4650$

veh $145-71-72-73-74-47-48=2=1=76=77-50-49=48=47=74-73-72-71-45$ load 300

veh $2 \quad 45-71-72-73-67-44=43-3=14=2=50=49=52=65=68=69=74-73-72-71-45$ load 300

veh $3 \quad 45-42=41=7=6=5=4=13-4=3=43=42-45$ load 295

veh $4 \quad 45-71-21=22=9=8=11=12=5-12=6-42=45$ load 300

veh $5 \quad 45-42=6-7=8-9=10=80=78=79-78=10-78=19=75-19=22-21=71-45$ load 300

veh $6 \quad 45-42-41=22=38=27=28-15-20=25-29=24-23-39-64=69=70-71-45$ load 300

veh $7 \quad 45-71-21=26=29=25=24=23=30-23=40=63=68=47=46-66=67-73-72-71-45$ load 300

veh $8 \quad 45-71-72-73-74-47-48-49-50=77=51-53=56-61=60=63-40-23=39=64=70-71-45$ load 300

veh $9 \quad 45-71=72=73=67=44=46=66=74=73-72=70=71=45$ load 125

veh $1045-71-70-69-68-65-52=62-61-60=58=59-58=57-58=55=54-55=56=61=62=65-68-69-70-71-45$ load 300

E16 opt $=3775$

veh $1 \quad 54-55-56=53=35-34=50-54$ load 260

veh $2 \quad 54-55=53=52=36-52=53=55-54$ load 145

veh $354=55=56-57=9=8=7=6=52=36-52=6=7=8=9=57-56=55=54$ load 255

veh $4 \quad 54-55-53-52-36-60-59-1-2=3=5=6-7-8=57=56-55-54$ load 300

veh $5 \quad 54-55-56-57-8-7=58=22=21-4=22-26-20=14=11-10=9-57-56-55-54$ load 295

veh $6 \quad 54-50-45-43=33-43=30-32-29=44=45=50=54$ load 300

veh $7 \quad 54-50-45=43-45-44=49=48=11=10-9-57-56-55-54$ load 300

veh $854-55-56-57-9-10=51-10-11=12=47-13=17=16-17=14=19=15=18-15=16=47-46=31-46=47=13=12=48-49$ $=42=50-54$ load 295 
E19 opt $=3235$

veh $1 \quad 27-23-22=20-4-19-5-6-16=45-46=47=51=40=9=14=15-14=9=40=51=47=46-45=16-6-5-19-4-20=22-23-27$ load 300

veh $2 \quad 27-23-13-42=49=43=48=39-48=16-18=49=13-23-27$ load 300

veh $327-23-22=19-5-17=18=16-48=45=46-47=14=15-14=47-46=45=48-16=18=17-5-19=22-23-27$ load 300

veh $4 \quad 27-26-36-35-38-44=41=50=42=13=23=27$ load 300

veh $5 \quad 27=26=28-29=11=10-11=12=54-12=34=55-34=33=31-29=30-2-3-21=25=24=27$ load 300

veh $6 \quad 27-26-36-35-38=61=60-61=62=63=59-56=33-31=32-31=29=28=25-24=22=23-27$ load 300

veh $727-26-36-35=44=53=57-53=58=60=44=38-37=62-63=37=38=35=36=26-27$ load 300

E20 opt $=2825$

veh $1 \quad 42=41=40=39-40=47=48-47=46=44=42$ load 145

veh $2 \quad 42-43=38-36=34=35-34=32=33-32=13-32=31=30-43-42$ load 300

veh $342-43-30=27=29=28=27-29=11=4=10-4=5=7-5=51-45-44-42$ load 290

veh $4 \quad 42-44-46=45=11=12=26-12=30=43-42$ load 260

veh $542-43-30-31-25=24=14=25=26=24-25=31=36=37-36=38=41-42$ load 295

veh $6 \quad 42-44-46=50=49-23-56=9=1=3=2=54=52=51-45-44-42$ load 290

veh $7 \quad 42=43=44=45=51=50=53-55-1=2=8-6=3-1=55=53=52-51-45-44-42$ load 255

E21 opt $=3730$

veh $1 \quad 25-22-24-29-31-34=36=37-36=38=39-38=40=35=34-31-29-24-22-25$ load 300

veh $2 \quad 25-26=28=14=15-14=16-18=20-18=17=19-17=16=18=21=23=22-25$ load 295

veh $325-26-28-12-53=52=51-52=13=47=33=13=53-12=28-26-25$ load 300

veh $425-26-28-12=53=54=55=52-55=56=57-50=9-50=57=10=56=54=11=14=22-25$ load 300

veh $5 \quad 25=22=21-23=24=29=30-29=27=28-27=26=25$ load 230

veh $6 \quad 25-22=24-29=31=32=42-32=33-13=12=11-12-28-26-25$ load 300

veh $725-22-24-29-31-34-36-38=7-8-6-5-1=46=40-41=42=43=44=45=46=3-2=44-43=45=41=40-35=36-34=31-29$ $-24-22-25$ load 300

E24 opt $=3510$

veh $1 \quad 69-96-8=10-12=44-2=1-2=44=45=6-9=43=42-70-69$ load 300

veh $269-70-42-40-47=46-37-50=4=5=3=2=56=58=57-58=53-54-52=51=4=6=47=40-42-70-69$ load 295

veh $369-70-74-39=40-47-6-45=3=53=54=55-59=60-48=49=38-41-39=74-70-69$ load 300

veh $4 \quad 69-70-74-39-41-38-49=37=50=51=55=59=57=54=52=5-4-6=9=10=43=8=96-69$ load 300

veh $5 \quad 69=96=11=7=94=93=7=92=97-92=93-94=95-96-69$ load 215

veh $6 \quad 69-70-74-73-72-64-75=76=62-90-61=76=63-76=26=25=28-25=75=64-72-73=74-70-69$ load 300

veh $7 \quad 69-70=42=40=41=38=29-30=64=72=41=39=73=72=71=70-69$ load $290 \quad 69=70=74=71=65-66=68=67-68$

$=95=96-69$ load 235

F04 opt $=3485$

veh $1 \quad 51-56=55=4-55=56-51$ load 180

veh $2 \quad 51-52-53-54=32=8-10-11-7=28-7-6=17=16-17=20-27=26=25-26=29=33-30=31-53-52-51$ load 600

veh $351-52-50-49=43-44=42-38=36=35=34=25=24-23=22=21-22=19-18=21=20=27=28=29-33=32=31=53-52-51$ load 600

veh $451-52=50=30=33=34-35=24=23=36=37=38=42=41=40-41-15-39=42=43=44=46=48=47=46=45-46-48$ $=49=50-52-51$ load 600

veh $5 \quad 51=52=53=54=5=55=4-55=5=54=53=52=51$ load 350

veh $6 \quad 51=56=57=63=62-63=64=70-66=69=68-69=67-69=68-69=66-70=64=63=62-63=57=56=51$ load 515

veh $751=57=59=60=61-60=62=59=58-59-62=64=65=69=67-69=65=64=62-59=58-59=62=60=61-60=59=57=51$ load 445

F08 opt $=3705$

veh $150-49=48-42=40=39=15-16=36=35-36=37=34=2-34=3-34-37=38=39=18-39-38=41-42-48-49-50$ load 565

veh $250=51=54-55-57=56=33=32=63-32=4=45=46=49=50$ load 520

veh $350-49-46=47-46=44=5-12-6=43=47=48=42=41=40=51-50$ load 400

veh $4 \quad 50-51-54-55=53-52=20=19=52=21-52=53=54-51-50$ load 495

veh $5 \quad 50-51-54=55=57=59=60=67=68=74-73=21=68-67=64-65=66=61=58-62=33-62=58=59-60=53-54-51-50$ load 580

F12 opt $=3395$

veh $121=20=18=17=16=19=21$ load 85

veh $221=22=35=36=34=29=28-29=24-46=47=44-47=41=23=21$ load 600

veh $321-19=18-20=15=14=43-14=7=15=6=64-72-70-67=39=40=6=17-18-19-21$ load 600

veh $4 \quad 21-19-16-17=38=39-40=67=65=66-68-69-2=8-30=32=34=33=12=50-12=10-9=31=32-37=36-37=39-38=22$ $=16=18-19-21$ load 600

veh $5 \quad 21-23=24=46-45=25=48=26=28=27=13=49-53-50=11=3-11=73-11=10=9=8=30-32=37=35-22-21$ load 600 


\section{E. Figures}

This section presents different analyses about the time that the components of a branch-and-price require. Every page depicts three figure groups presenting data for one of the eight instance groups egl-e1- $n$ to egl-e4- $n$ and egl-s1- $n$ to egl-s4- $n$ with $n \in\{\mathrm{a}, \mathrm{b}, \mathrm{c}\}$. First, those instances solved to optimality are mentioned. The first figure group shows the evolution of the lower bound values over time for different instances and pricing relaxations and correspond to Figure 4 in the main paper. Thereafter, the number of branch-and-bound nodes solved and the type of branching decision taken impacts which and how often a particular algorithmic component is invoked (related to Figure 5). The last figure group is concerned with the effort for solving the pricing problem (corresponding to Figure 6 of the main paper). 
Solved to optimality: egl-e1-a, egl-e1-b

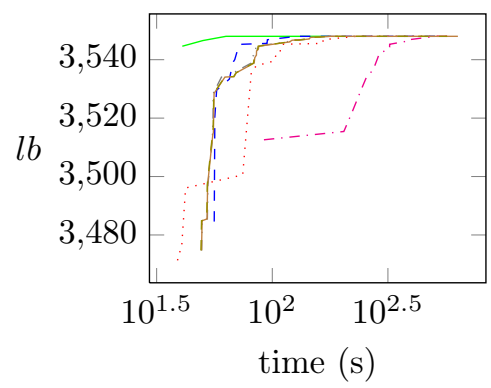

(a) egl-e1-a

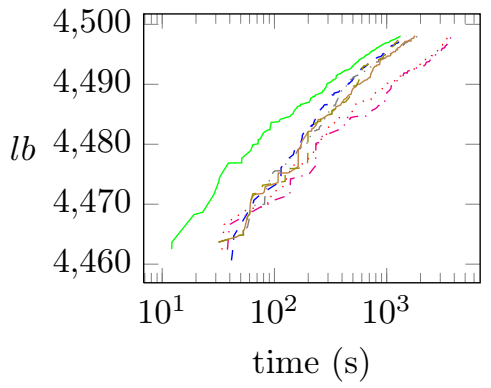

(b) egl-e1-b

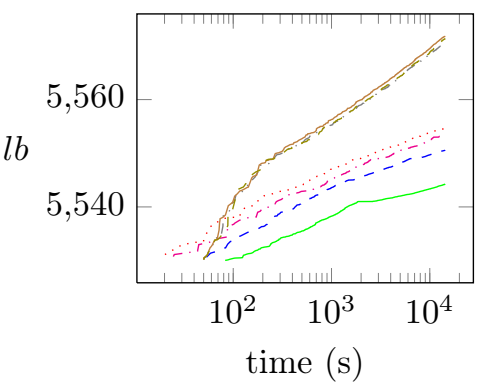

(c) egl-e1-c

Figure 7: Lower bounds over Time

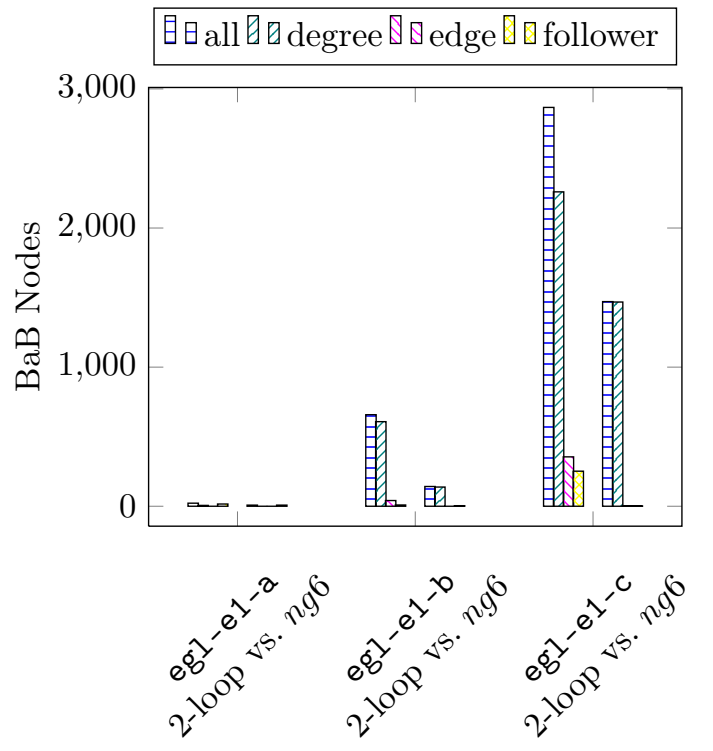

(d) Num. B\&B Nodes

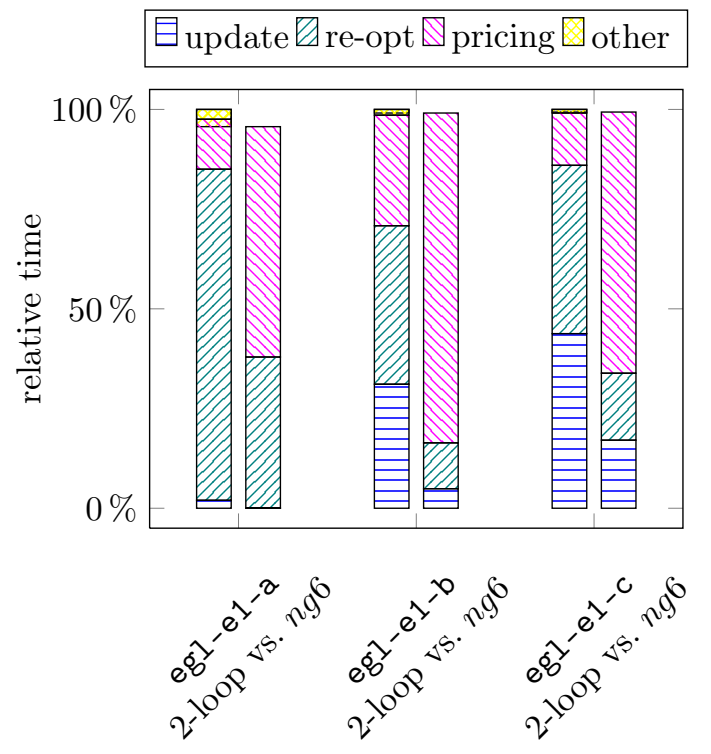

(e) Relative Times

Figure 8: Number of Branch-and-Bound Nodes/Decisions and Relative Times spent in Components

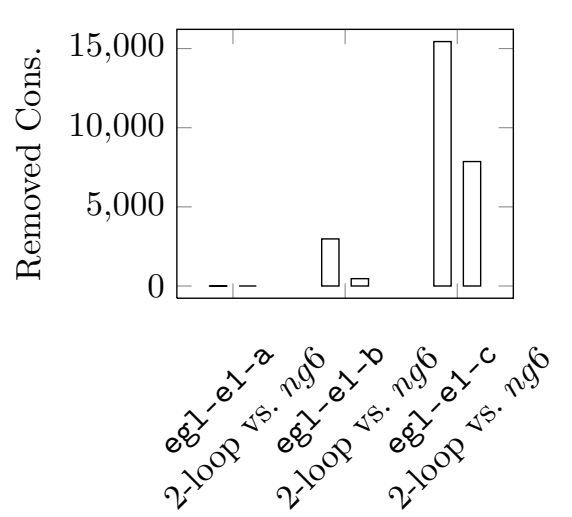

(a) Removed Constraints

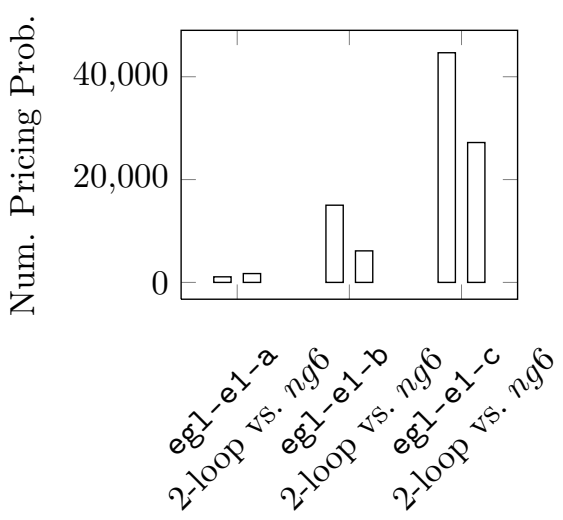

(b) Num Pricing Problems
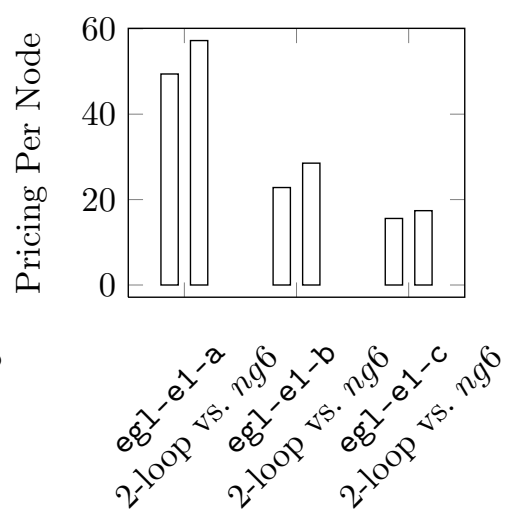

(c) Pricings Per Node

Figure 9: Number of Pricing Problems overall/per Node 
Solved to optimality: egl-e2-a

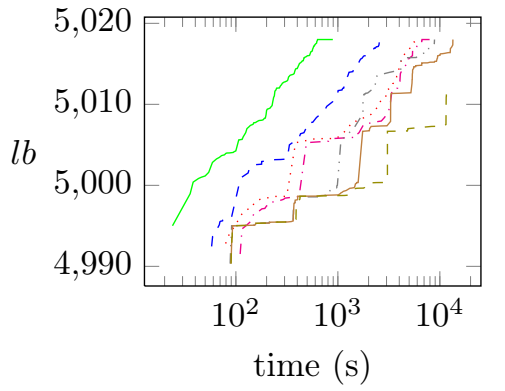

(a) egl-e2-a

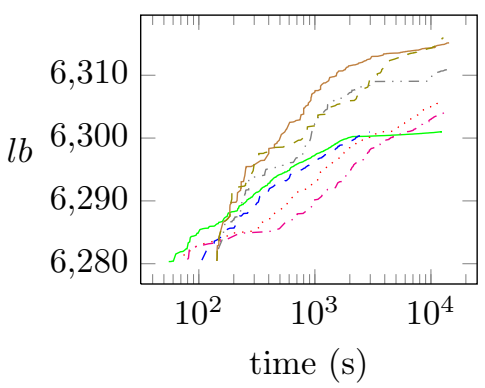

(b) egl-e2-b

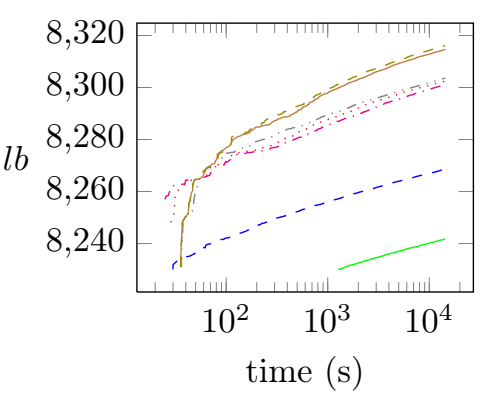

(c) egl-e2-c

Figure 10: Lower bounds over Time

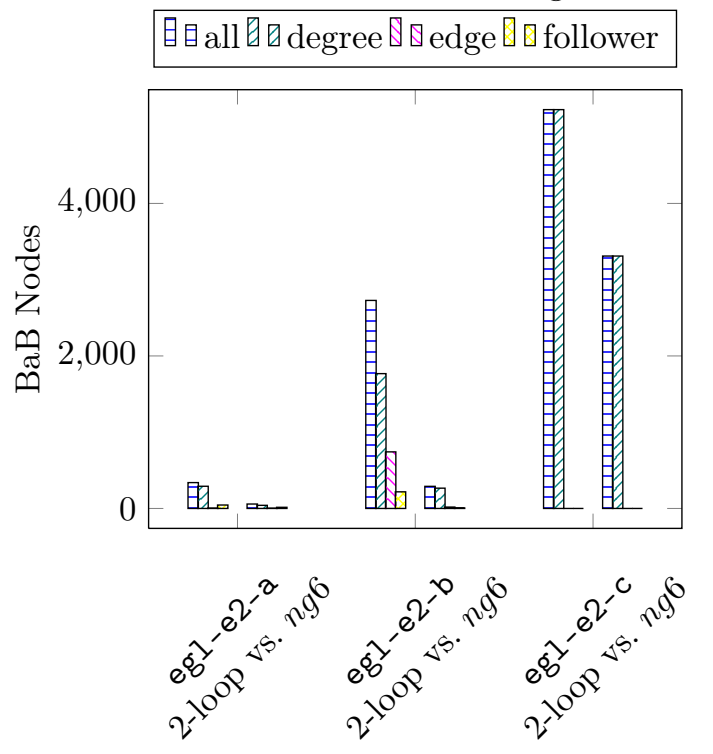

(d) Num. B\&B Nodes

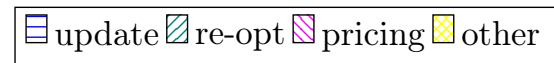

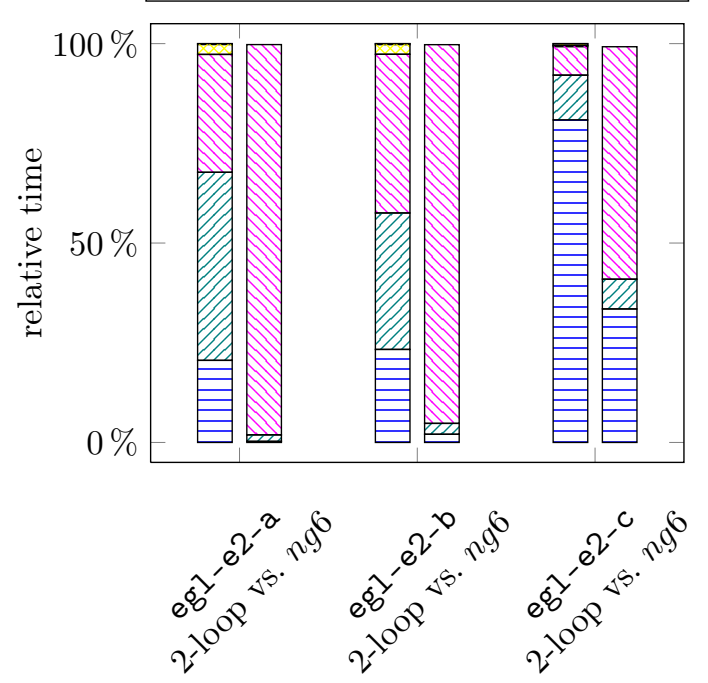

(e) Relative Times

Figure 11: Number of Branch-and-Bound Nodes/Decisions and Relative Times spent in Components

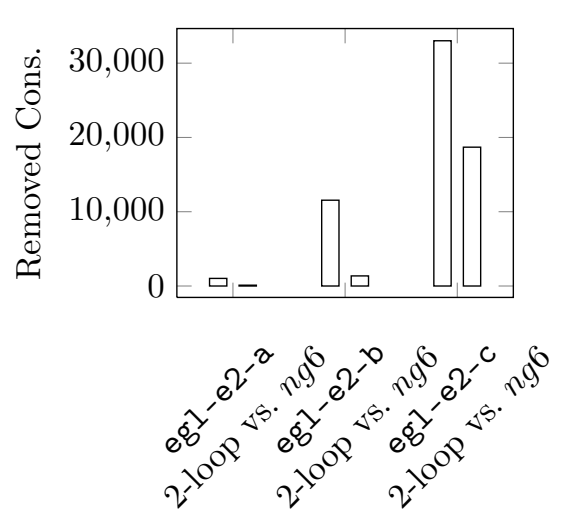

(a) Removed Constraints

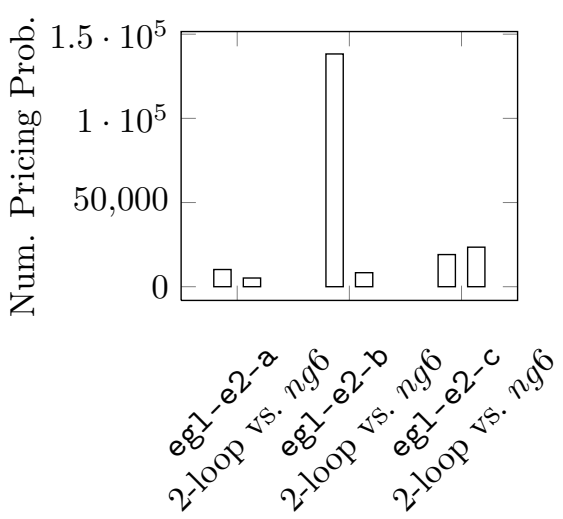

(b) Num Pricing Problems
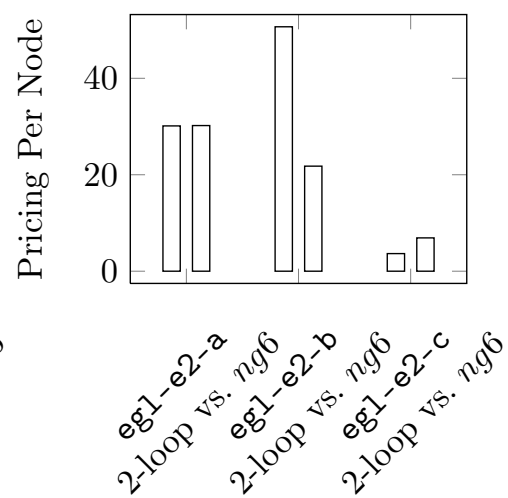

(c) Pricings Per Node

Figure 12: Number of Pricing Problems overall/per Node 
Solved to optimality: egl-e3-a

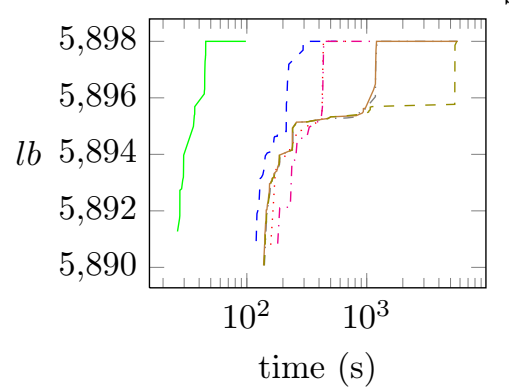

(a) egl-e3-a

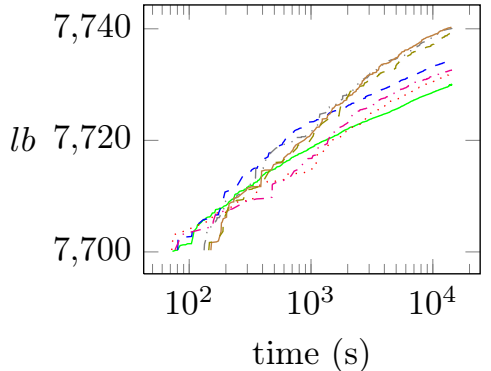

(b) egl-e3-b

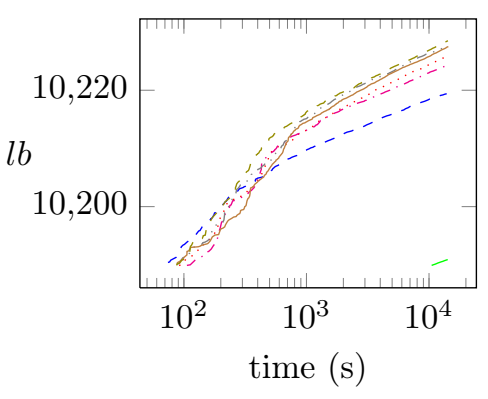

(c) egl-e3-c

Figure 13: Lower bounds over Time

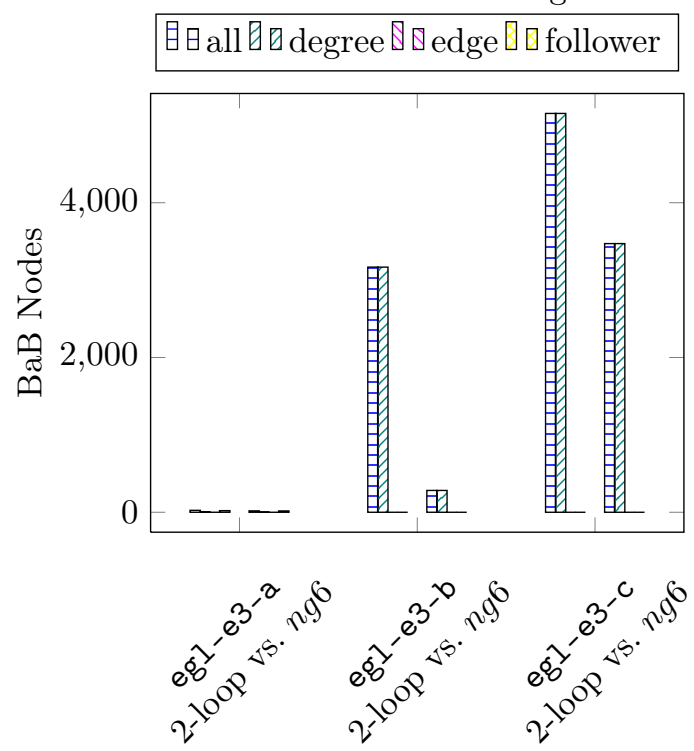

(d) Num. B\&B Nodes

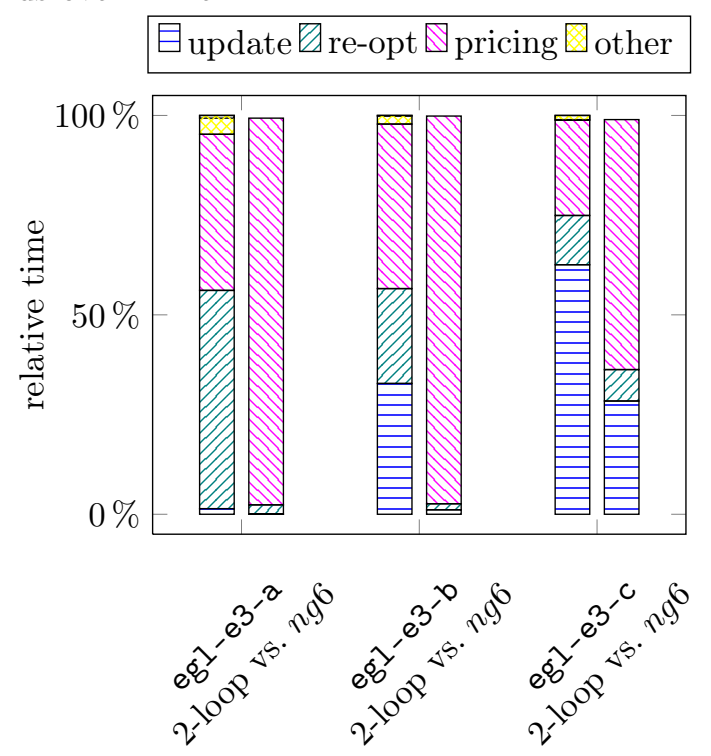

(e) Relative Times

Figure 14: Number of Branch-and-Bound Nodes/Decisions and Relative Times spent in Components

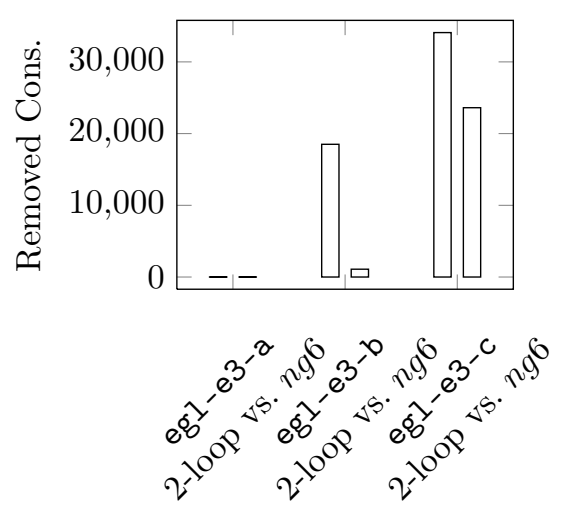

(a) Removed Constraints

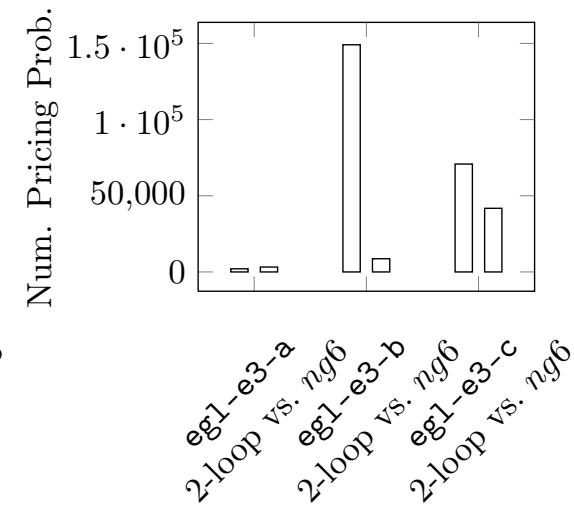

(b) Num Pricing Problems
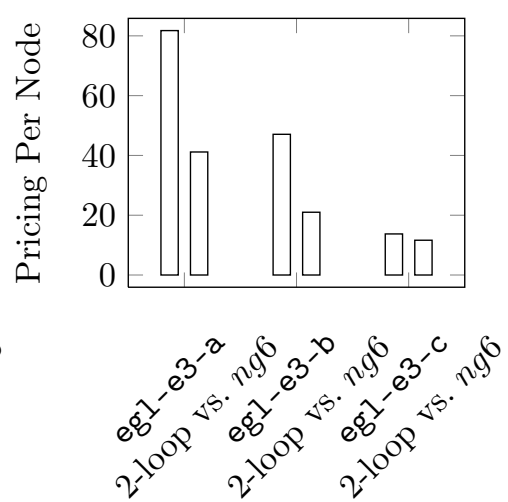

(c) Pricings Per Node

Figure 15: Number of Pricing Problems overall/per Node 
Solved to optimality:

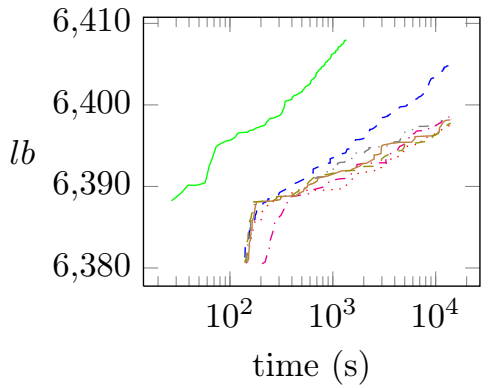

(a) egl-e4-a

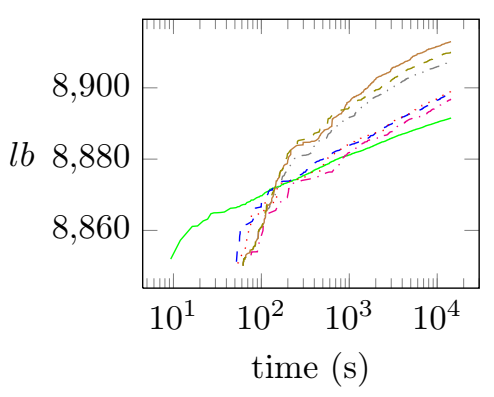

(b) egl-e4-b

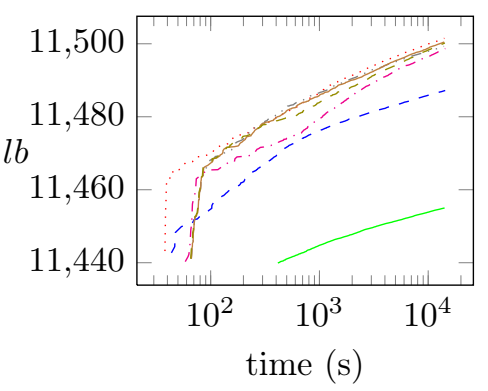

(c) egl-e4-c

Figure 16: Lower bounds over Time

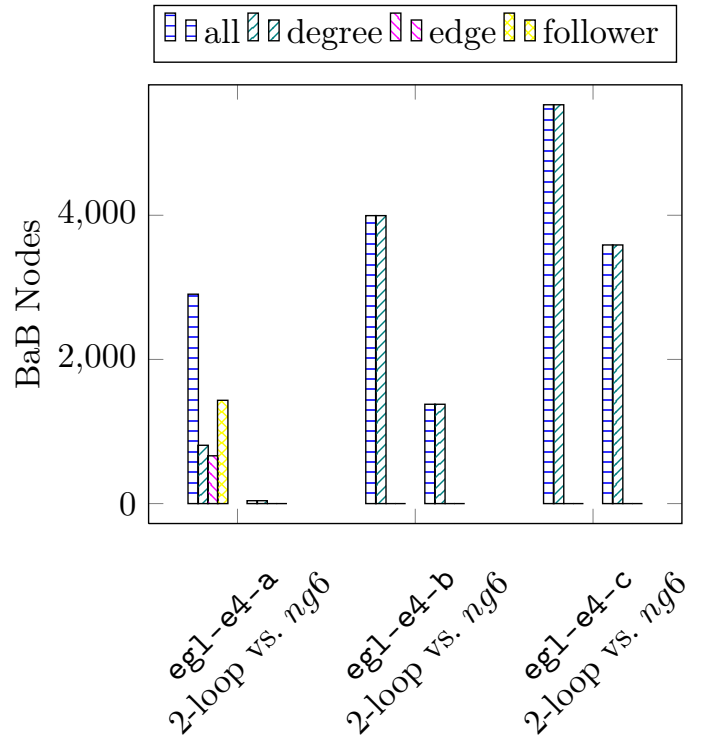

(d) Num. B\&B Nodes

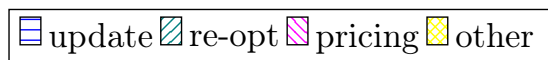

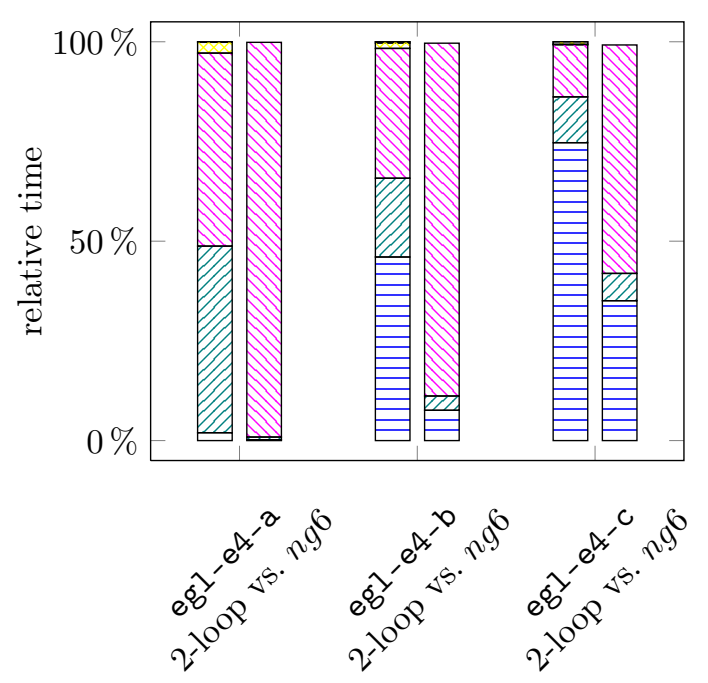

(e) Relative Times

Figure 17: Number of Branch-and-Bound Nodes/Decisions and Relative Times spent in Components

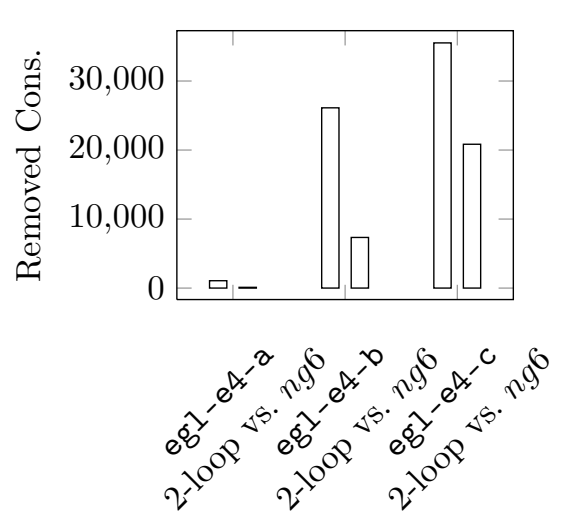

(a) Removed Constraints

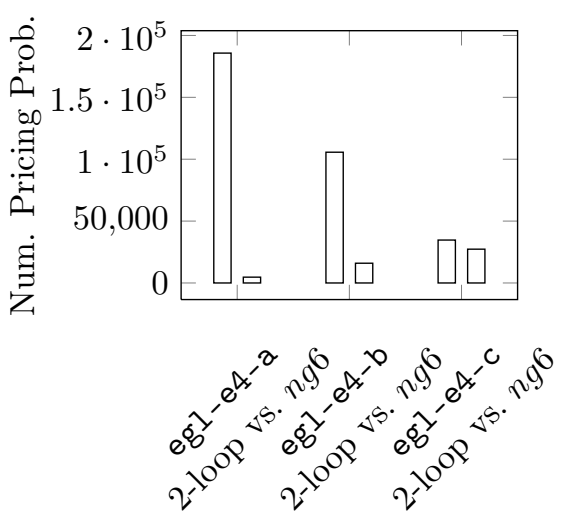

(b) Num Pricing Problems
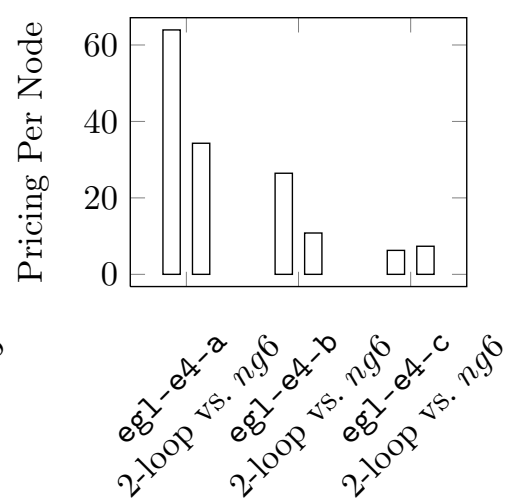

(c) Pricings Per Node

Figure 18: Number of Pricing Problems overall/per Node 
Solved to optimality: egl-s1-a

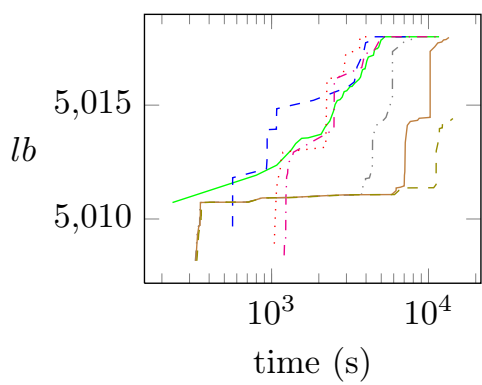

(a) egl-s1-a

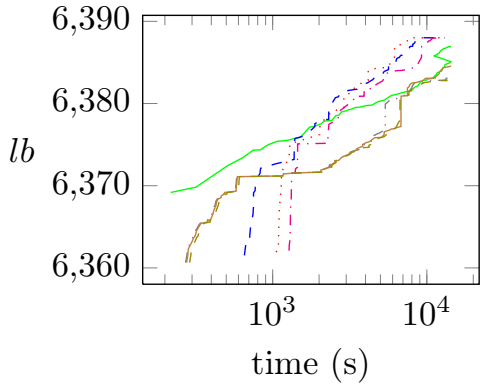

(b) egl-s1-b

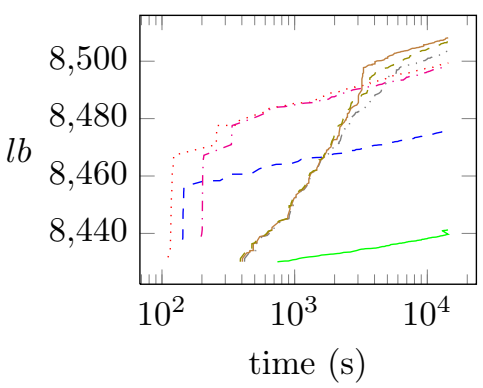

(c) egl-s1-c

Figure 19: Lower bounds over Time

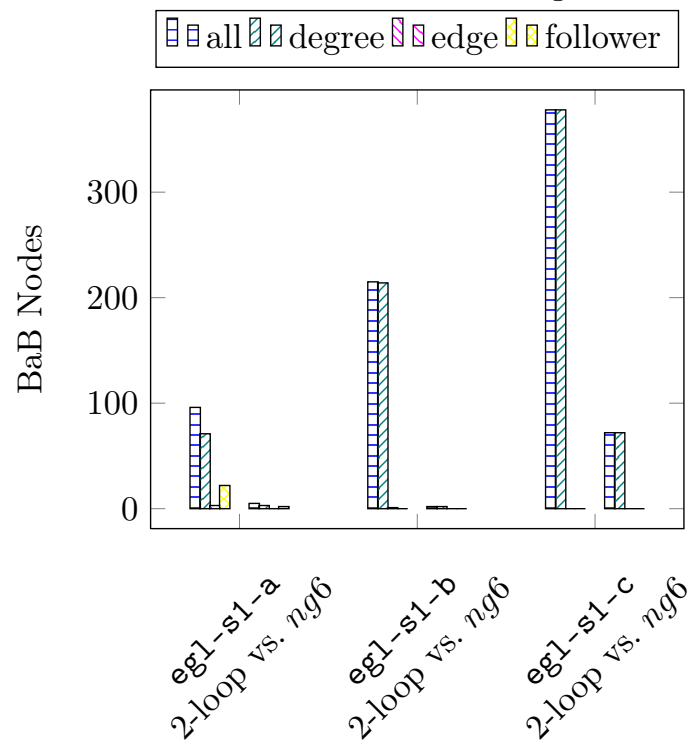

(d) Num. B\&B Nodes

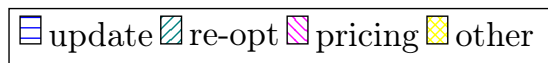

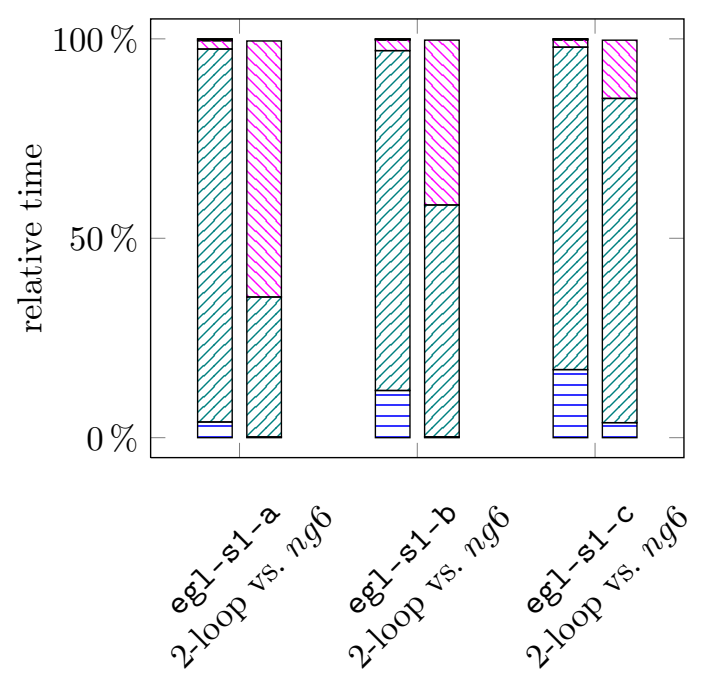

(e) Relative Times

Figure 20: Number of Branch-and-Bound Nodes/Decisions and Relative Times spent in Components

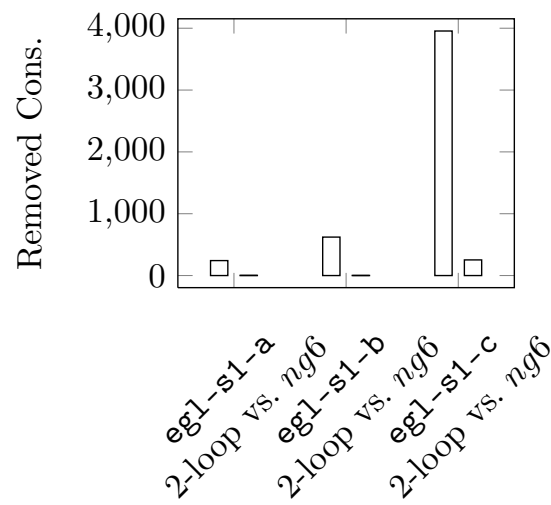

(a) Removed Constraints

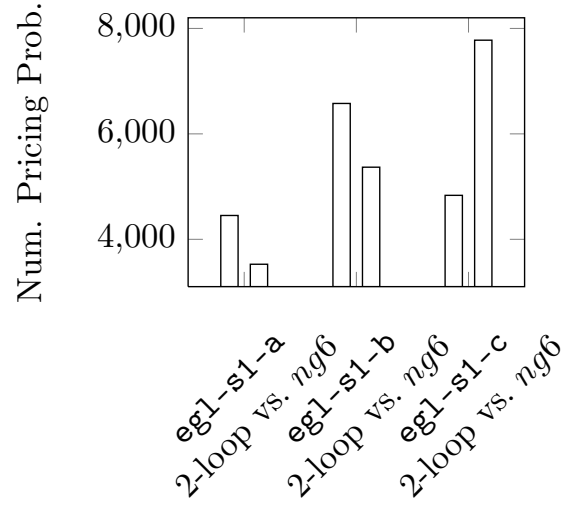

(b) Num Pricing Problems
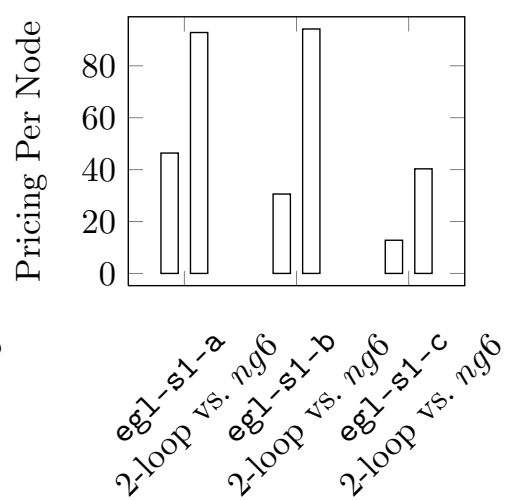

(c) Pricings Per Node

Figure 21: Number of Pricing Problems overall/per Node 


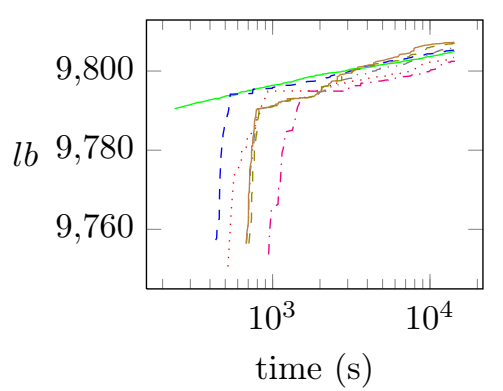

(a) egl-s2-a

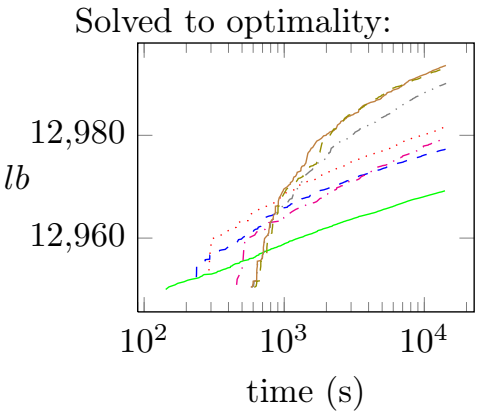

(b) egl-s2-b

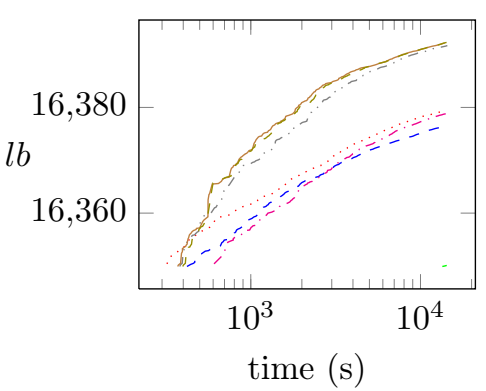

(c) egl-s2-c

Figure 22: Lower bounds over Time

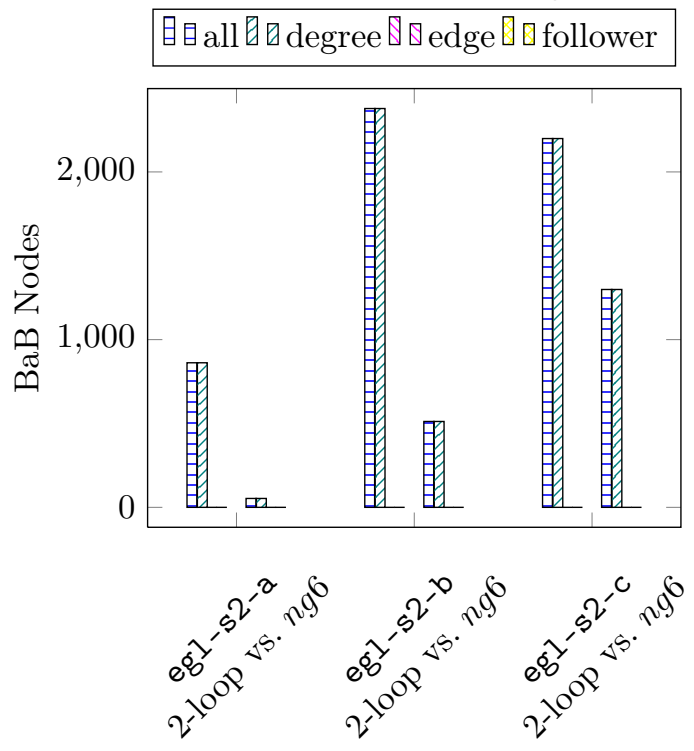

(d) Num. B\&B Nodes

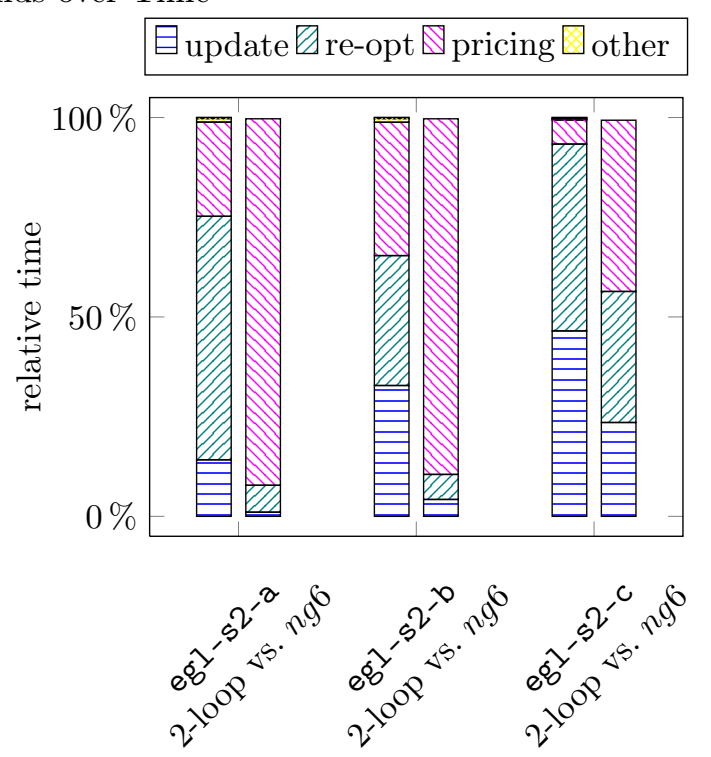

(e) Relative Times

Figure 23: Number of Branch-and-Bound Nodes/Decisions and Relative Times spent in Components

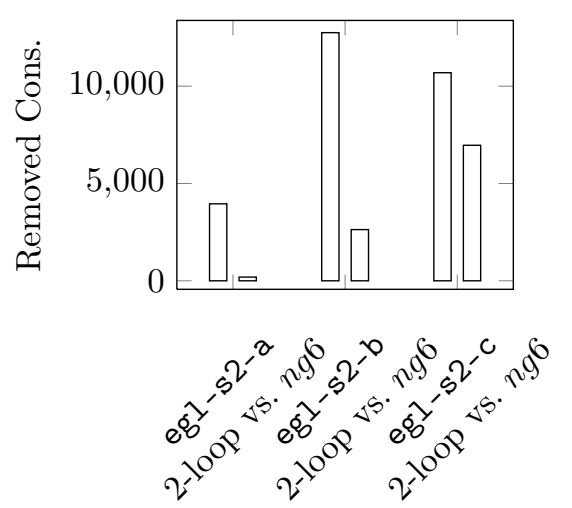

(a) Removed Constraints

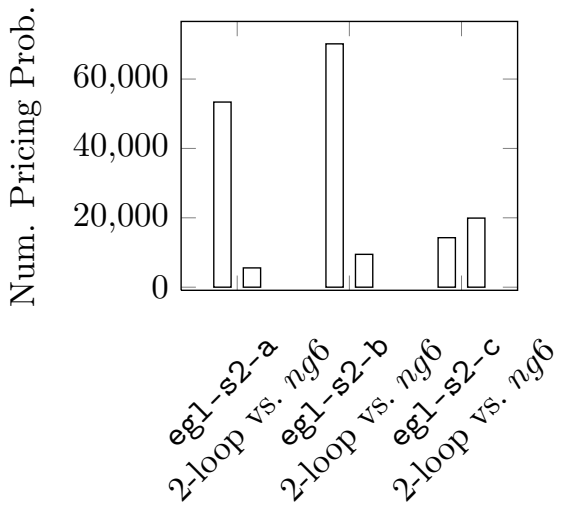

(b) Num Pricing Problems
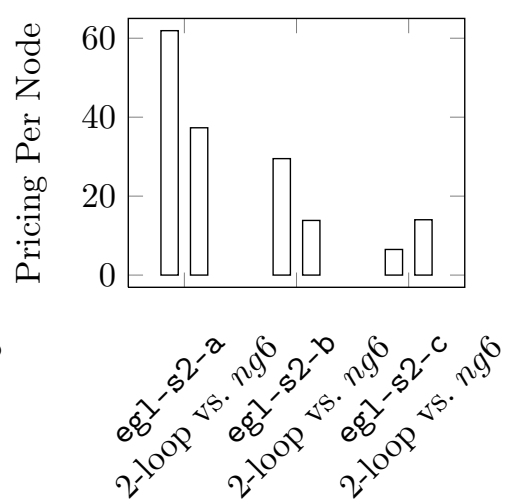

(c) Pricings Per Node

Figure 24: Number of Pricing Problems overall/per Node 
Solved to optimality:

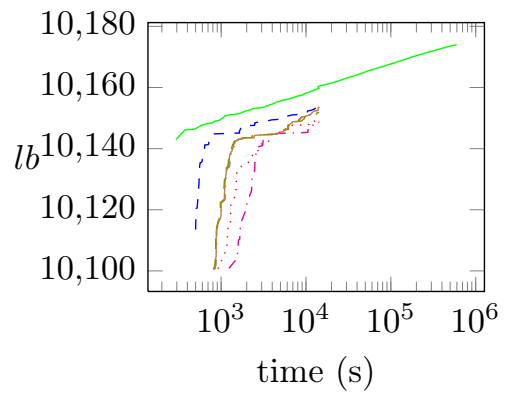

(a) egl-s3-a

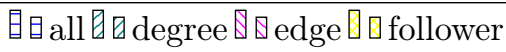

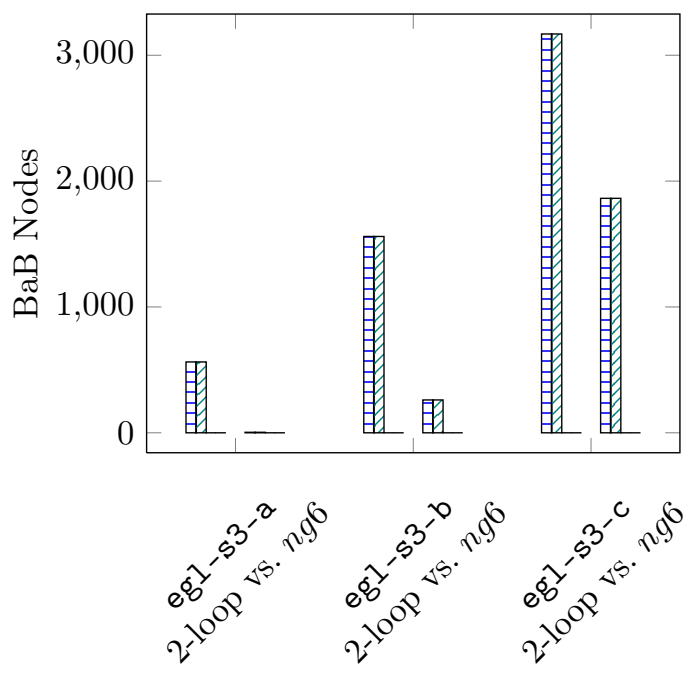

(d) Num. B\&B Nodes

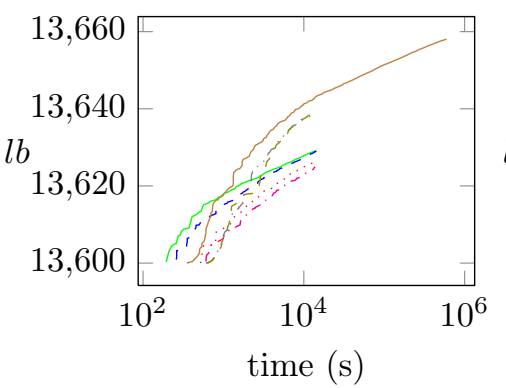

(b) egl-s3-b

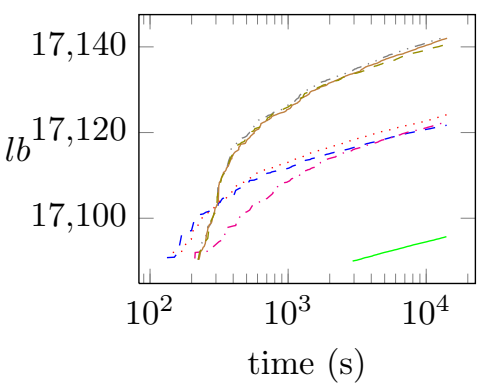

(c) egl-s3-c

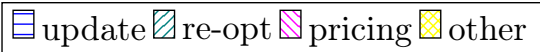

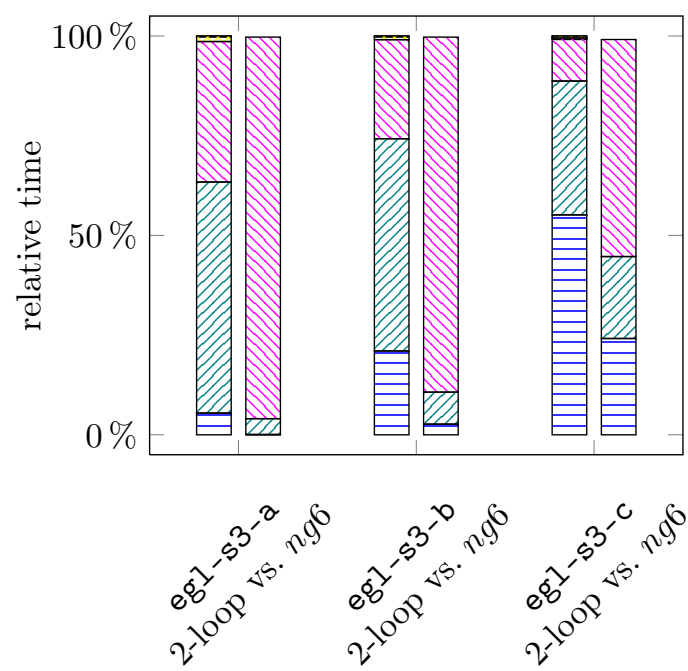

(e) Relative Times

Figure 25: Number of Branch-and-Bound Nodes/Decisions and Relative Times spent in Components

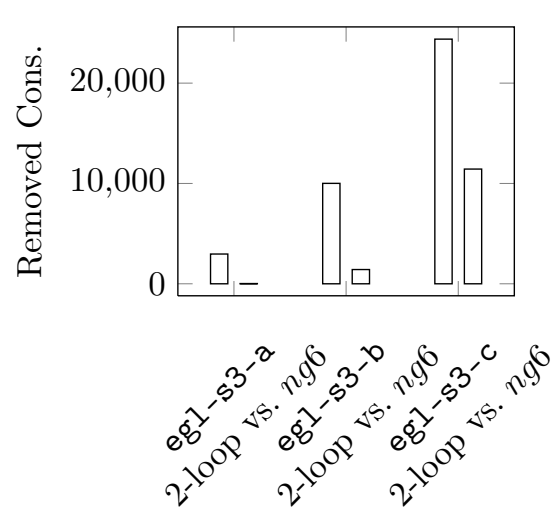

(f) Removed Constraints

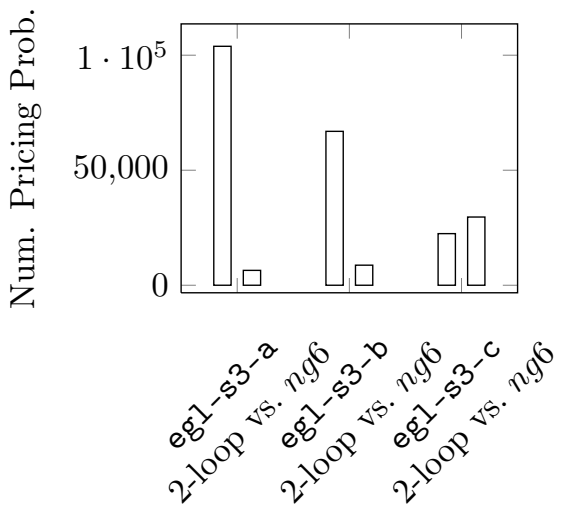

(g) Num Pricing Problems
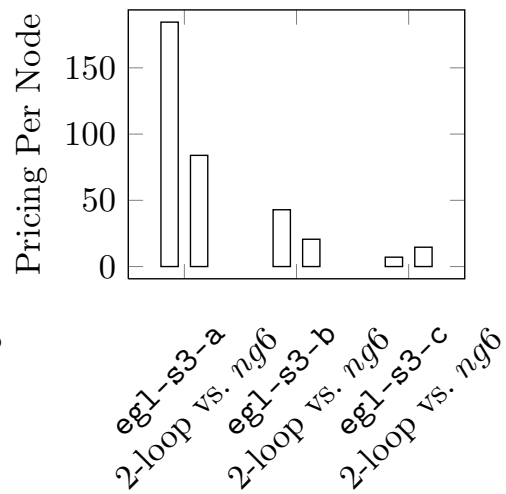

(h) Pricings Per Node

Figure 26: Number of Pricing Problems overall/per Node 


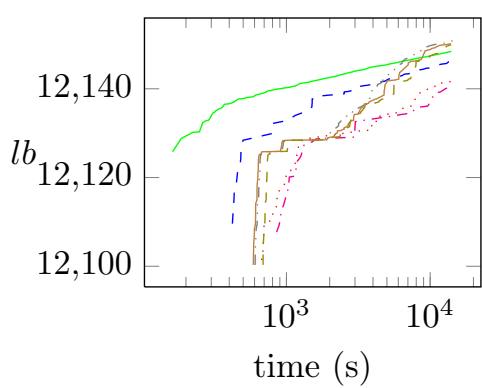

(a) egl-s4-a

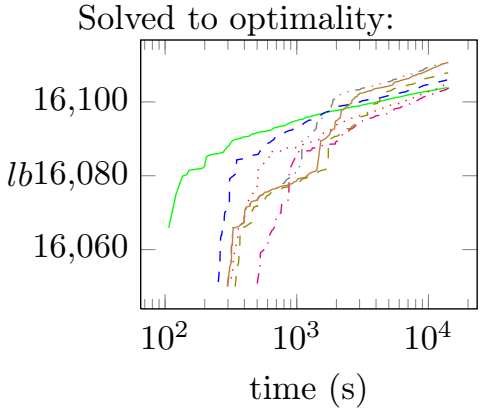

(b) egl-s4-b

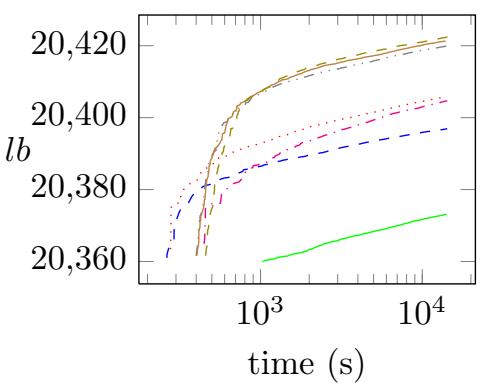

(c) egl-s4-c

Figure 27: Lower bounds over Time

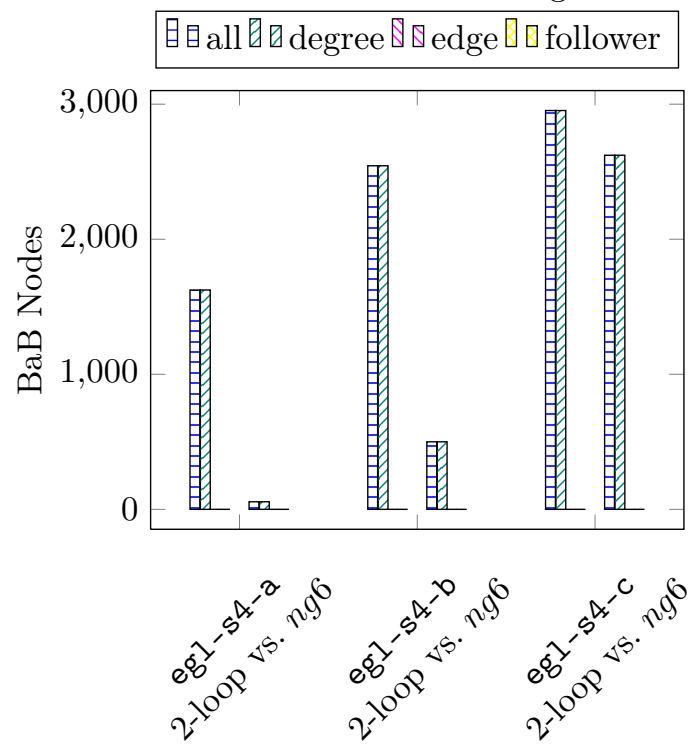

(d) Num. B\&B Nodes

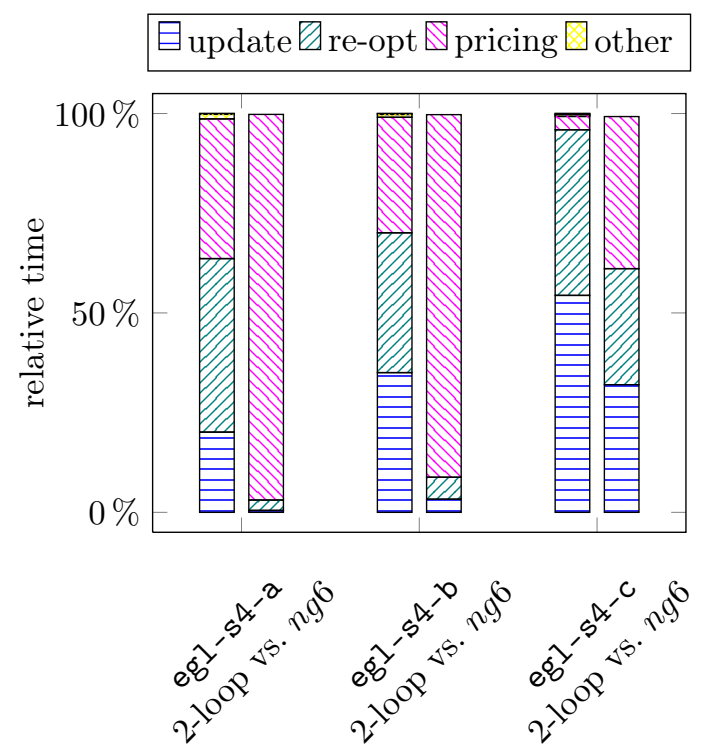

(e) Relative Times

Figure 28: Number of Branch-and-Bound Nodes/Decisions and Relative Times spent in Components

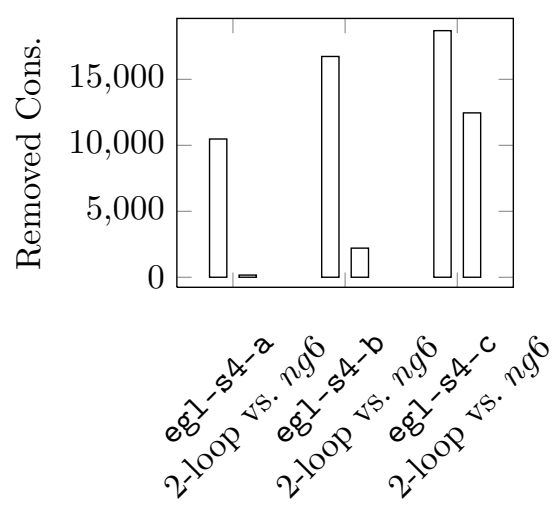

(a) Removed Constraints
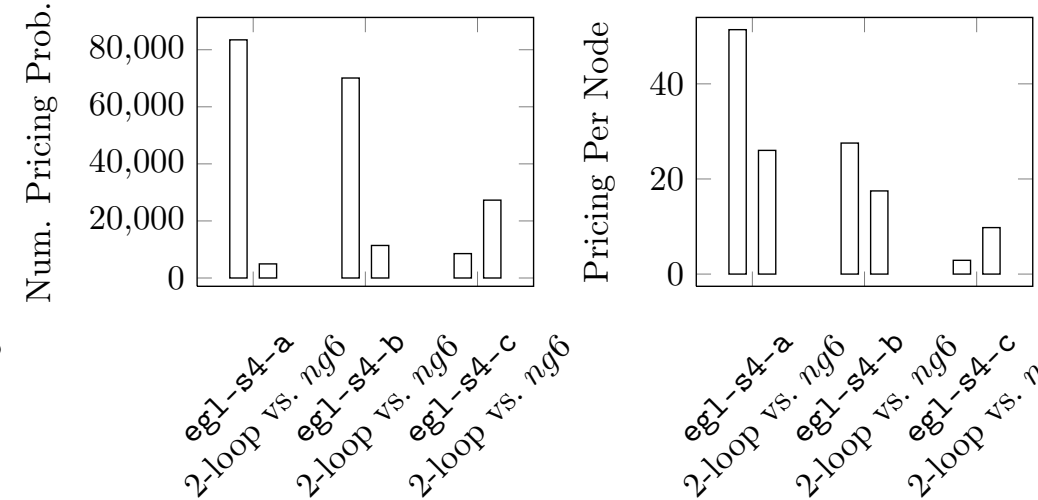

(b) Num Pricing Problems

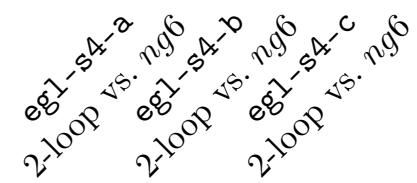

(c) Pricings Per Node

Figure 29: Number of Pricing Problems overall/per Node 